\title{
Chronic Stress, Depressive Symptoms and Peripheral Vascular Dysfunction: Fundamentals, Mechanisms, Sex Disparities, and Determinants of Integrated Outcomes
}

Shyla Christine Stanley

Follow this and additional works at: https://researchrepository.wvu.edu/etd

\section{Recommended Citation}

Stanley, Shyla Christine, "Chronic Stress, Depressive Symptoms and Peripheral Vascular Dysfunction: Fundamentals, Mechanisms, Sex Disparities, and Determinants of Integrated Outcomes" (2015). Graduate Theses, Dissertations, and Problem Reports. 6714.

https://researchrepository.wvu.edu/etd/6714

This Dissertation is protected by copyright and/or related rights. It has been brought to you by the The Research Repository @ WVU with permission from the rights-holder(s). You are free to use this Dissertation in any way that is permitted by the copyright and related rights legislation that applies to your use. For other uses you must obtain permission from the rights-holder(s) directly, unless additional rights are indicated by a Creative Commons license in the record and/ or on the work itself. This Dissertation has been accepted for inclusion in WVU Graduate Theses, Dissertations, and Problem Reports collection by an authorized administrator of The Research Repository @ WVU.

For more information, please contact researchrepository@mail.wvu.edu. 


\title{
Chronic Stress, Depressive Symptoms and Peripheral Vascular Dysfunction: Fundamentals, Mechanisms, Sex Disparities, and Determinants of Integrated Outcomes
}

\author{
Shyla Christine Stanley \\ Dissertation submitted to the School of Medicine at West Virginia University in partial \\ fulfillment of the requirements for the degree of:
}

Doctor of Philosophy in Cellular and Integrative Physiology

Jefferson C. Frisbee, Ph.D.- Research Advisor
Robert W Brock, Ph.D.- Program Director

Dissertation Committee

David P. Siderovski, Ph.D., Committee Chair

Kevin T. Larkin, Ph.D.

Peter R. Giacobbi, Ph.D.

Alexandre C. d'Audiffret, M.D.

Paul J. Marvar, Ph.D., External Member

Cellular and Integrative Physiology Program

Morgantown, West Virginia

2015

Key Words: Depression, cardiovascular disease, endothelial dysfunction, metabolic syndrome, sex disparities

Copyright 2015 Shyla Christine Stanley 


\section{ABSTRACT \\ Chronic Stress, Depressive Symptoms and Peripheral Vascular Dysfunction: Fundamentals, Mechanisms, Sex Disparities, and Determinants of Integrated Outcomes}

\section{Shyla Christine Stanley}

Chronic, irresolvable psychological stress, a major contributor of depressive disorders, has been identified as a key factor involved in the association between the prevalence/severity of depressive symptoms and the concurrent development of cardiovascular disease (CVD). Furthermore, there are marked differences in the incidence and pathology of these diseases between males and females, although the mechanisms involved are unclear. The purpose of this dissertation is to investigate the effects of chronic stress/depression on behavioral and vascular function in rodents in order to elucidate the underlying mechanisms linking depressive symptom severity with cardiovascular development/outcome. The unpredictable chronic mild stress (UCMS) model is well recognized in behavioral studies as a translationally appropriate animal model for generating depressive symptoms in rodents that accurately reflect human clinical characteristics, including learned helplessness and anhedonia (Willner et al., 1997; Yalcin et al., 2008). This model is based on the fundamental concept that chronic exposure to uncontrollable exogenous stressors will ultimately lead to depressive disorders (Mineur et al., 2003). This model will be used to compare functional outcomes (relative to behavior and CVD risk) between males and females, as well as the effects of this chronic stress regimen in animals with pre-existing cardiovascular risk.

The differential effects of UCMS on depressive symptom severity and vascular function on male and female rodents were initially performed using the BALB/cJ mouse (Chapter 1 of this dissertation). The following experiments (Chapters 2 and 3) utilized male and female lean and obese Zucker rats (LZR and OZR) as a comorbid animal model of metabolic syndrome (MetS). Furthermore, ovariectomized (OVX) female LZR and OZR were utilized in order to further investigate sex specific mechanisms involved in cardiovascular and behavioral outcomes to UCMS. The OZR develop similar symptoms of MetS observed in humans due to a genetic mutation in the leptin receptor gene that causes loss of satiety signaling, thus leading to chronic hyperphagia and the subsequent onset of obesity, hyperglycemia, insulin resistance, dyslipidemia, and moderate hypertension. MetS, which affects $34 \%$ of adults ( $\geq 20$ years of age) in the United States, is generally defined by the presence of a constellation of comorbid factors (abdominal obesity, insulin resistance, atherogenic 
dyslipidemia, pro-inflammatory/pro-thrombotic state) that are significantly associated with increased CVD risk (American Heart Association, 2014). The OZR also progressively develop non-atherosclerotic peripheral vascular disease in addition to severe vascular impairments, and therefore represents a relevant model of underlying CVD risk.

This dissertation addresses the differential sex-specific effects of UCMS-induced chronic stress on depressive-like behavioral severity and the severity of impaired vascular responsivity to endothelial dependent vasodilator stimuli, as well as the specific mechanisms underlying stress- induced vascular dysfunction, between male and female rodents. These functional outcomes were interrogated in male and female OZR (versus lean rodents, LZR) to determine the consequences of UCMS-induced chronic stress/depressive symptoms on rodents with pre-existing MetS. Finally, the effects of UCMS were investigated in OVX LZR and OZR to determine the implications of loss of female sex hormones on behavior and cardiovascular function in both healthy and obese female rodents.

Specific Aims:

Aim 1: To determine the impact of UCMS on vascular reactivity in mice of both sexes. It is hypothesized that UCMS will result in a more severe development of depressive symptoms in female mice (vs. males), but vascular function will be superior to that of males.

Aim 2: To determine if the protective effect against UCMS-induced vasculopathy in females is altered in rats with pre-existing cardiovascular disease risk factors and/or following OVX. It is hypothesized that vascular function in female animals will no longer be protected from UCMS-induced impairments in animals with comorbid CVD risk factors or in animals having undergone OVX.

Aim 3: To determine the impact of pharmacological interventions on chronic stress/depressive symptoms and vascular function in male animals with pre-existing cardiovascular disease risk factors. It is predicted that improving CVD risk and vascular function with drugs will improve behavioral symptoms/corticosterone levels. 


\section{DEDICATIONS}

To everyone who has supported me during the last few years and provided unwavering encouragement to never give up.

To Doug, Mom, and Dad 


\section{ACKNOWLEDGEMENTS}

My experience in graduate school has been a life-altering journey that I could not have successfully completed without the help of my friends and family. First of all, I want to thank Mom and Dad. Your endless support and love gave me the motivation to keep moving forward, despite the many challenges and battles that came along. As for Mom, the everyday phone conversations with you helped me cope with being so far away from home. You always listened to me bicker and vent, and gave me words of encouragement when I needed them most. Because of you, I was able to keep my sanity during the particularly stressful, rough times. So thank you, Mom, my best friend. I have to mention my baby boy, Doug. I would have been so alone without him and his unconditional love; he is the best companion I could have asked for. And of course, Steven, my great supporter, the eternal optimist, the most unselfish and loving man I know, I owe a part of this Ph.D. to you and your extraordinary patience and selfless commitment to always be there for me and help in any way possible. Thank you for sticking with me and never criticizing or doubting me. Not even once.

A special thanks to a past member of the Frisbee Laboratory, Josh. You always tried to reach out to me and pull me out of my dark little corner. Without your guidance, I would have truly been lost. Thank you for being such a genuine, thoughtful friend and teacher to me. I needed that desperately.

Throughout the years, I have hit a lot of hurdles that left me on the ground, so badly wounded that I really did not see how I could possibly get up again. But I did, and I owe that entirely to one of the most exceptional, inspirational people I have ever met. Thank you, Jeff, for being my number one supporter and always, without a doubt, cheering me on and giving me the strength to move forward, whether leading me by the hand or dragging me by the leg; you never gave up on me. Somehow you managed to bring out a smile even on the most dismal of days. Thank you for taking your chances on a scared little girl holding a teddy bear in your office on that fateful day you offered me a position in your lab. It has been the most rewarding experience of my life and I will never forget it.

Of course, thank you to my committee members, Dr. Siderovski, Dr. Larkin, Dr. Giacobbi, Dr. Marvar, and Dr. d'Audiffret, for your support and encouragement. And a special thanks to Dr. Brock, for your guidance and support over the past few years. I appreciate it so much. 
To everyone that has been there for me and supported me through the years, thank you from the bottom of my heart. 


\section{TABLE OF CONTENTS}

Chronic Stress, Depressive Symptoms and Peripheral Vascular Dysfunction: Fundamentals, Mechanisms, Sex Disparities, and Determinants of Integrated Outcomes

i

Abstract

Dedication

Acknowledgements

Table of Content

Abbreviations

Introduction ii

iv

$\mathrm{v}$

vii

viii

1

Chapter 1: Protective Effect of Sex on Chronic Stress and Depressive Behavior Induced Vascular Dysfunction in Balb/cJ Mice 26

Chapter 2: The Role of Female Sex Hormones in Modulating Stress-Induced Vascular Dysfunction in Lean and Obese Zucker Rats 64

Chapter 3: Interventions Blunting Systemic Inflammation and Oxidant Stress are Required to Improve Vascular Function and Depressive Symptoms in Metabolic Syndrome 102

Discussion 130 
Metabolic Syndrome

Nitric Oxide

Prostacyclin

Prostaglandin $\mathrm{H}_{2}$ 


\section{INTRODUCTION}

\section{$\underline{\text { Cardiovascular Disease (CVD) }}$}

Cardiovascular disease (CVD), the leading cause of morbidity and mortality in the United States and worldwide, causes substantial reductions in the quality of life, and accounts for significant health-related expenditures (World Health Organization, 2014; Go et al., 2014). An estimated 17.5 million people die each year from CVD, accounting for more than 30 percent of global deaths (American Heart Association, 2012). The total cost of CVD in the United States (US) was estimated to be $\$ 320$ billion dollars in 2012, including both direct (healthcare related) and indirect (lost productivity) expenses (The American Heart Association, 2012).

CVD refers to a group of insidious pathological disease states that are defined by either: 1) a weakening/abnormality of the heart or, 2) an inadequate blood supply to tissues/organ systems due to narrowing of the aorta or aortic branches, peripheral arteries (peripheral vascular disease), cerebral vessels (stroke), or coronary arteries (coronary heart disease) (The American Heart Association, 2012). Current treatment options for CVD vary, but generally focus on reducing the work load on the heart by improving blood pressure/cardiac function, or alleviating areas of restricted blood flow in the vasculature though pharmacological/surgical procedures (Mahoney et al., 2010; Ouriel et al., 2001). Well-documented pathologies associated with increased risk of the progression of CVD include, diabetes, hyperlipidemia, high blood pressure, arthritis, and obesity (Ford and Mokdad, 2008; Cannon, 2007; Steinberg et al., 2008). The American Heart Association (AHA) and the American College of Cardiology (ACC) assessed the frequency of comorbidities and the percentage of the

population of adults 65 years of age and older is shown in figure 1. The most common comorbid conditions for three index cardiovascular conditions: ischemic heart disease, heart failure, and stroke, were hypertension, hyperlipidemia, diabetes, and arthritis (Arnett et al., 2014). Furthermore, the prevalence of two or more chronic conditions is estimated to be present in more than one quarter of US adults (Ward and Schiller et al., 2010). 

AHA/ACC/HHS Clinical Practice Guidelines

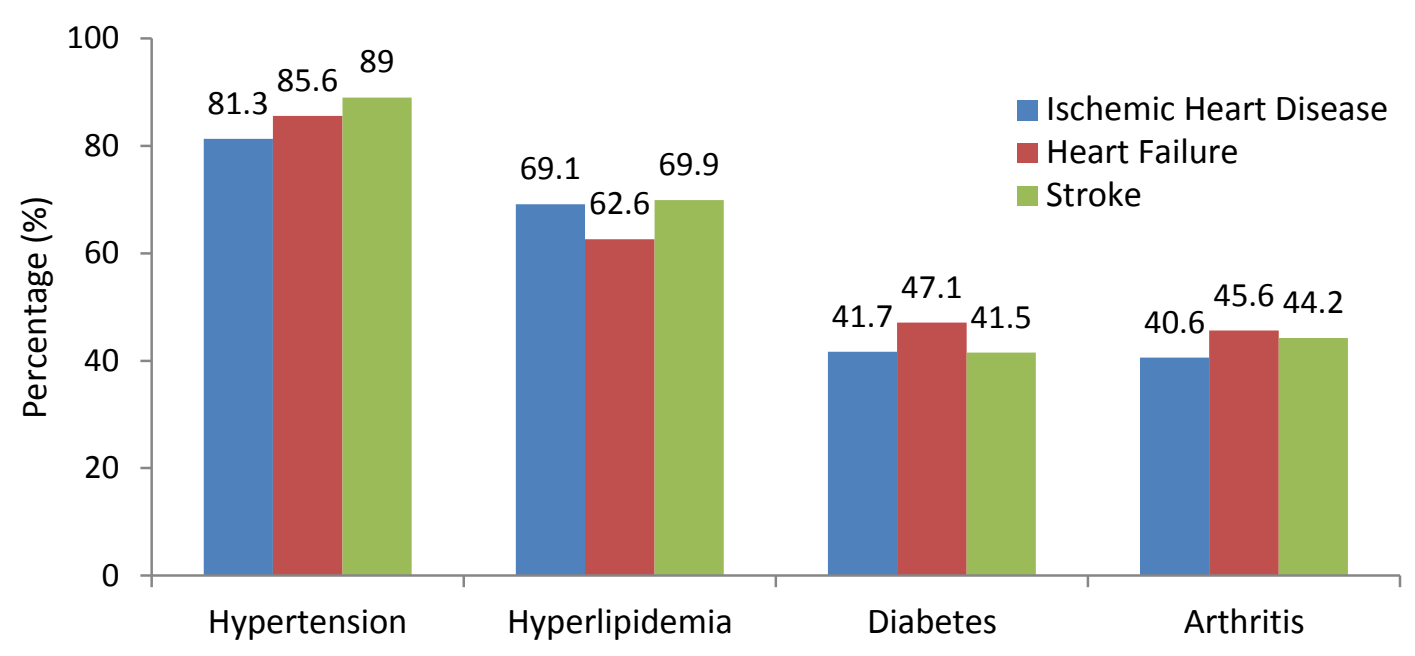

As the global prevalence of these systemic pathologies continue to rise (Barbero et al., 2015), they are becoming more frequently presented as comorbidities, representing an integrative disease state associated with worsened health outcome (Kannan et al., 2008). Such a constellation of risk factors, specifically, abdominal obesity, insulin resistance, elevated triglycerides, and low high-density lipoprotein cholesterol, is defined as the metabolic syndrome (MetS). MetS is used to identify patients at high risk of CVD, type II diabetes, and overall mortality (Alberti et al., 2009). It has been hypothesized that obesity is the defining pathology that contributes to the evolution of MetS as well as some of the other components.

A critical effect of MetS is a reduction in the perfusion of blood flow to peripheral tissues that gradually causes a decline in function and progressive loss of tissue viability, thereby severely impacting the ability to exercise (Zhou $\mathrm{Z}$ et al., 2014). The failure to deliver and distribute blood to match metabolic demand is associated with alterations in structure and compliance of the vasculature, and this also affects hemodynamic stress on the heart as well as constrains functional hyperemia (Chantler and Frisbee, 2015). Functional hyperemia is defined as an increase in tissue/organ system perfusion in response to metabolic demand. These effects could be caused by mechanisms that alter vasodilator signaling, vasoconstrictor forces that compete with vasodilation, or structural changes in vessel wall dynamics (Frisbee et al., 2009). However, structural alterations in the vasculature are not 
always associated with CVD and both animal and human studies have demonstrated that vascular disease can manifest in the absence of atherosclerotic plaques and lesions (Frisbee et al., 2011; Goodwill et al., 2009). Instead, extensive evidence has demonstrated that maintenance of perfusion distribution and functional hyperemia is dependent on endothelial function and adrenergic activity. Epidemiological studies have demonstrated that endothelial dysfunction precedes any observable clinical sign of vascular disease and has been reported to predict early subclinical stages of atherosclerotic disease (Grover-Páez and Zavalza-Gómez, 2009). This is clearly evident in resistance arterioles of pre-hypertensive patients, which show impaired endothelial-dependent vascular reactivity to dilator stimuli without visible structural changes far in advance of complications of hypertension, and is also observed with aging, obesity, smoking, and other conditions in the absence of elevated blood pressure (Schiffrin and Touyz, 2004). In fact, all of the underlying pathologies of MetS negatively impact the endothelium, and impaired endothelium-dependent vasodilation of the brachial artery is observed in patients with MetS (Chantler and Frisbee, 2015).

In addition, overactivity of the sympathetic nervous system has been implicated in virtually all CVD, and subsequent adrenergic hyperactivity on vascular smooth muscle cells and endothelial cells increases vascular tone, reduces vasodilation, and decreases blood flow (Zhou et al., 2014). It is clearly evident that any alteration to vascular reactivity will greatly influence bulk flow of blood as well as regulation of blood pressure and peripheral resistance (Hart et al., 2009). The consequential imbalance between endothelium-derived vasodilatation and sympathetic activity under pathophysiological circumstances may cause vasoconstriction, leukocyte adherence, platelet activation, thrombosis, impaired coagulation, vascular inflammation, pro-oxidation, and progression of atherosclerosis. The role of endothelial dysfunction and adrenergic signaling will be addressed below.

\section{$\underline{\text { Endothelial Dysfunction }}$}

Broadly speaking, endothelial dysfunction is defined as an impaired endothelial dependent vasodilator response characterized by reduced nitric oxide (NO) bioavailability. This pathway is shown in figure 2 . NO is produced as a byproduct of catalytic conversion of NADPH and O2-dependent oxidation of 1-arginine to 1citrulline mediated by eNOS. This pathway is activated by acetylcholine (ACh), bradykinin (B), and physical forces (shear stress), as well as autacoids, prostaglandins, and platelet (serotonin and adenosine diphosphate) as 
well as that cause eNOS to dimerize and sequester iron, enabling generation of high-affinity binding sites for arginine and the cofactor tetrahydrobiopterin $\left(\mathrm{BH}_{4}\right)$, which allow electron transfer from the reductase-domain flavins to the oxygenase-domain heme (Zou et al., 2002). This reaction produces NO. NO rapidly diffuses into adjacent smooth muscle cells, where it activates guanylyl cyclase - cGM pathway and induces vasodilation by multiple mechanisms: inhibition of calcium entry into the cell and decrease intracellular calcium concentrations; activation of $\mathrm{K}+$ channels, which leads to hyperpolarization and relaxation; stimulation of a cGMP-dependent protein kinase that activates myosin light chain phosphatase (Hart et al., 2011). Under normal conditions, NO is one of the most potent vasodilators in the endothelium and is essential for maintaining normal homeostatic balance within that vasculature, potentiating conditions of vasodilatation, low permeability, anticoagulation, and inhibition of proinflammatory actions of leukocytes and platelets (Kelly et al., 2004).

Figure 2: NO Pathway in Endothelial Cells and Smooth Muscle Cells

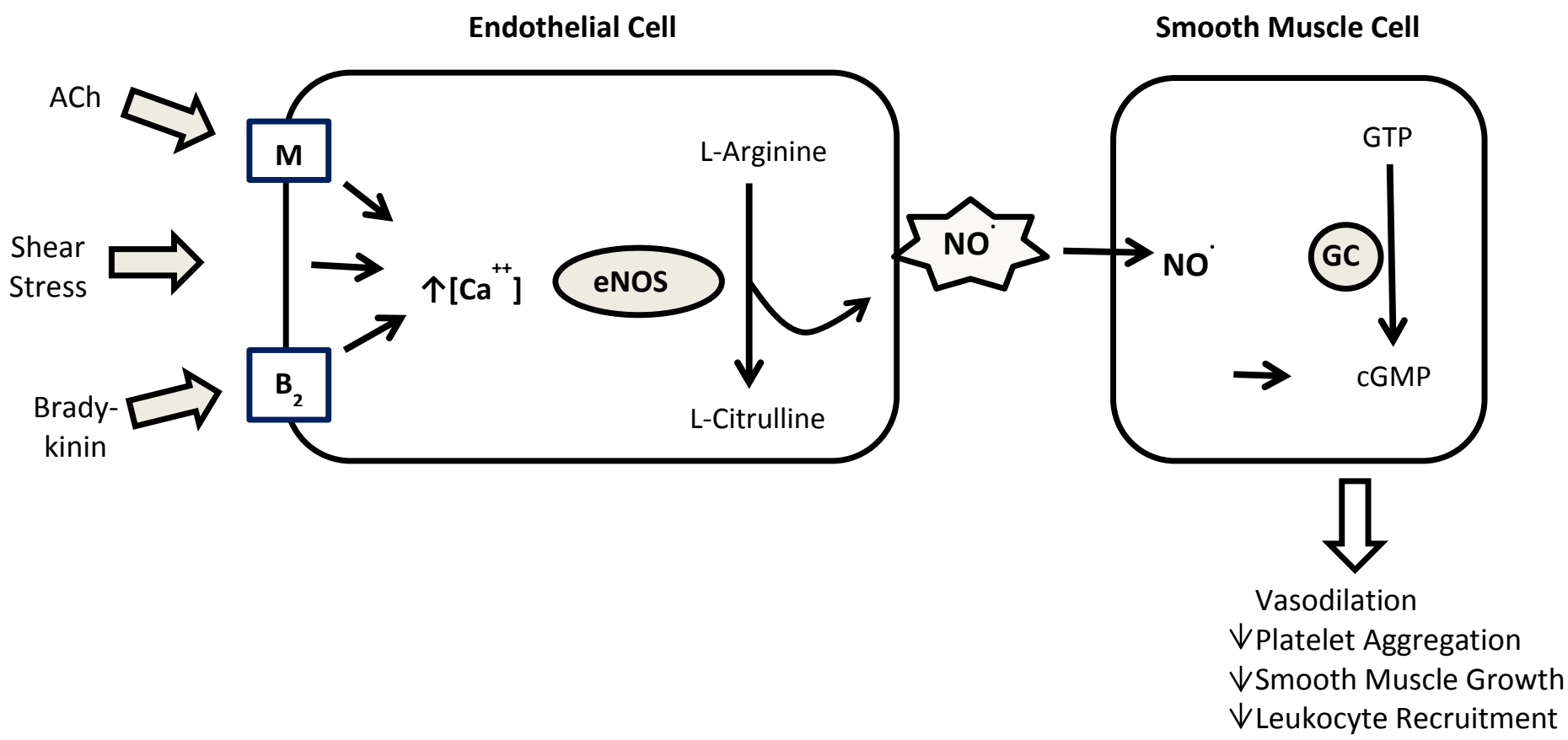


Endothelial dysfunction can be measured in different pathological conditions by vasodilator reactivity to either pharmacological (e.g. infusion of endothelium-dependent agonists, such as acetylcholine) or physiological (e.g. reactive hyperemia) challenge. A widely used clinical method employs flow mediated dilation (FMD), a noninvasive in vivo measurement of endothelium-dependent vasodilation to shear stress in the brachial artery. Numerous studies have demonstrated consistent reports of impaired FMD associated with endothelial dysfunction and prognosis of CHD (Chan et al., 2003), untreated hypertension (Perticone et al., 2001), angina (Neunteufl et al., 2000) PVD (Gokce et al., 2003) and chronic stress/depression (Sherwood, 2005). The most important relationship that will be the focus of this dissertation is the bidirectional relationship between chronic stress/depressive disorders and the development of CVD and CV risk factors.

\section{$\underline{\text { CVD and Depression }}$}

The influence of psychological factors on the multifaceted pathophysiology of chronic diseases has been an emerging field of behavioral cardiology (Mansura et al., 2015). Stress is a well-established trigger of mood disorders, such as depression; stressful life events are one of the strongest predictors of onset and relapse, while chronic stress exposure is associated with greater symptom severity and prevalence (Kim et al., 2007). Decades of research investigating the relationship between chronic stress/depression and adverse CV events has established that chronic stress/depression is a powerful risk factor for CVD morbidity and mortality and is also predictive of more severe prognosis of cardiovascular events, including myocardial infarction (MI), coronary artery disease, cardiomyopathies, and atherosclerosis (Rutledge et al., 2006; Pizzi et al., 2010). Furthermore, depression predicts first CVD events even among otherwise healthy individuals, especially in women, and increases CVD incidence 1.5 to 6 fold (American Heart Association, 2011). Importantly this has been reported to be independent of traditional CVD risk factors, including hypertension, insulin resistance, and total serum cholesterol (Penninx et al., 2001).

\section{$\underline{\text { Sex Differences }}$}

Sex disparity in the prevalence, clinical presentation and prognosis of both CVD and depression has clearly been demonstrated in clinical and epidemiological studies (Hart et al., 2010). Premenopausal women have 
a lower incidence of CVD compared to age-matched men until approximately the age of menopause, at which time a significant rise in the incidence of CVD events, as well as mortality rates, surpasses that of men (MollerLeimkuhler, 2007). In addition, classic CVD risk factors such as, hypertension, smoking, depression, and obesity, also correlate more to overall CVD risk in women (Vaccarino et al., 1999; Bello et al., 2004; Kenachaiah et al., 2004; Polk et al., 2005) compared to men. Surprisingly, pre-menopausal women are less likely to suffer from CVD (Kessler et al 2003; 1993), but are concurrently two times as likely to be afflicted with depressive disorders compared to men (Lloyd-Jones et al., 2010). This trend diminishes around the age of menopause as depression incidence drops down to rates comparable to those of men. Current literature supports an association between estrogen and the progression of depressive symptoms and comorbid diseases (Lloyd-Jones et al., 2010; Daniel et al., 2005) relative to general CV protection and mechanisms related to NO processing and maintenance of vascular homeostatic balance.

\section{Unpredictable Chronic Mild Stress (UCMS)}

Research opportunities that focus on specific mechanisms underlying the critical relationship between chronic stress/depression and the progression of vascular disease have been greatly facilitated with the emergence of translationally valid animal models. Several preclinical models of depression have been developed over the past decade for use in behavioral research, theoretically based on the following parameters: similarity of the model to the etiology and progression of clinical depression; ability of the model to recapitulate anatomical, biochemical, neurophysiological, and behavioral features of human pathology; and similarity to the outcomes of common interventions/treatments in humans (Strekalova et al., 2011). The most widely used preclinical models of depression in behavioral research include the learned helplessness model (Porset, 2005), the early life stress model (Willner, 1997), the social defeat model (Grippo, 2005), and the unpredictable chronic mild stress (UCMS) model (Isingrini et al., 2012). Learned helplessness is a paradigm based on the observation that animals develop deficits in motivation, cognitive and reward behaviors following repeated unavoidable and uncontrollable shocks. Models of early life stress involve prenatal stress, early postnatal handling and maternal separation, and have been beneficial in studies of chronic stress-induced effects on epigenetics and early life 
development. The social defeat stress model is designed to create a state of submission and anxiety in an inferior animal via imposition of a physically superior aggressive animal.

The model used for the experiments of this dissertation is the unpredictable Chronic Mild Stress (UCMS) model. The UCMS model involves repeated and randomized exposure of daily mild stressors, causing gradually progress to a cumulative stress response. In these means, animals are exposed to randomized mild environmental and social stressors each day for 8 weeks (Willner, 1997). A crucial distinguishing factor that enhances the translational relevance of this model to chronic stress in humans lies in the high degree of unpredictability and uncontrollability of the stressors encountered over an extended period, without imposing life-threatening or severe challenges on the animals. The UCMS model relies on the fundamental concept that chronic exposure to unpredictable, exogenous stressors ultimately leads to the development of depressive symptoms similar to that of clinical depression, including decreased responsiveness to rewards (anhedonia), changes in physical activity and investigative behavior (helplessness and despair), deterioration of the coat state and altered sexual activity (Willner, 1997). Furthermore, variable behavioral outcomes in rodents have been reported in UCMS studies. For example, a stress resilient phenotype that largely prevents depressive-like behavior changes following UCMS can develop, indicating differences in stress susceptibility (Bouzinova et al., 2012). Additionally, altered depressive behaviors can gradually be reversed by chronic, but not acute, treatment with certain antidepressants, demonstrating similar neurological effects of antidepressants in depressed patients. Lastly, this model is most fitting for this dissertation based on observations showing UCMS-induced depressive symptom severity in rodents is a powerful, independent predictor of endothelium-dependent vasomotor responses to pharmacological stimuli in aortic rings and peripheral arteries (Stanley et al., 2014; d'Audiffret et al., 2010). Specifically, the attenuated endothelial dependent vasodilator reactivity is associated with similar outcomes observed in patients with depression, including decreased NO bioavailability (Chrapko et al., 2004), and increased levels of circulating biomarkers for cellular adhesion, inflammation, and thrombosis (Rajagopalan et al., 2001; Dimopoulos et al., 2006). In addition, sex specific differences in outcomes following UCMS are observed, further increasing the value of this method for investigating sex disparity in chronic stress/depressive disorders and CVD pathology. Therefore, this dissertation will focus on mechanisms linking chronic stress/depression and 
the evolution of CVD and related vascular diseases, specifically those involved in compromising functional hyperemia and vascular reactivity.

Sympathetic-Adrenal-Medullay (SAM) Axis

The adrenergic system has been the focus of many research investigations into the pathogenesis of chronic stress/depression and CVD (Antonijevic, 2006). Hyperactivity of SAM axis is observed in depressed patients and persistent noradrenergic activity has been linked with negative outcomes in patients with CVD and CVD risk factors, including congestive heart failure (Francis et al., 1993) and diabetes (Ganguly et al., 1986). Most of these effects are initiated by SAM-induced release of catacholamines, norepinephrine (NE) and epinephrine (Epi). Central NE influence alertness, arousal, and reward systems; enhances corticotropin-releasing hormone $(\mathrm{CRH})$ release from the hypothalamus; and promotes sympathetic activity while attenuating vagal innervation. In the periphery, the adrenergic system innervates vascular smooth muscle cells and endothelial cells, strongly impacting vascular reactivity (Schreihofer et al., 2005; Frisbee, 2006; Esler, 2001). Any such alteration will greatly influence regional blood flow as well as regulation of blood pressure, vasomotor tone, and peripheral resistance (Hart et al., 2009). Studies in the OZR have confirmed that pre-existing CVD risk factor is associated with increased vasoconstrictor reactivity in response to adrenergic stimuli in both skeletal muscle and renal circulation (Naik et al., 2006) while intravenous infusion of $\alpha$-adrenoreceptor antagonists eliminated differences in arterial pressure in OZRs and significantly increased perfusion through these vascular networks compared to their lean controls. Together, these data suggest increased adrenergic response in a MetS model alters blood flow.

In recent years, several studies have demonstrated that sex-specific mechanisms moderate sympathetic neural and vascular control in humans. For example, estrogen binding to endothelial membrane estrogen receptors causes the release of NO from the endothelium (Duckles \& Miller, 2010), opposing the vasoconstrictor effects of sympathetic stimulation. Data previously published demonstrated an increase in vasoconstriction to phenylephrine in arteriolar vessels of male mice subjected to UCMS, while a similar vasoconstrictor response was observed in UCMS females only after inhibition of NOS activity with L-NAME (Stanley et al., 2014). This suggests that modulation of adrenergic vascular reactivity by endogenous NO under non-stressed conditions remains largely intact in females despite UCMS, but is diminished in males. The loss of NO actions on attenuating adrenergic 
sensitivity in conditions of chronic stress and reduced NO bioavailability (Hart et al., 2009; Puzserova et al., 2012) may be an underlying reason for coordination between mental stress- induced sympathetic activity and systemic peripheral resistance in young men, but not women (Hart et al., 2009). Furthermore, studies have demonstrated that women exhibit a blunted transduction of muscle sympathetic nerve activity (MSNA) into limb vascular tone following psychological stress, while men were more sensitive to the vasoconstrictor action of MSNA during the same stress challenge (Yang et al., 2013). Differences in neural-vascular coupling have been linked to adrenergic receptor sensitivity/density as well as effects of estrogen on NO bioavailability (Hart et al., 2009).

\section{Hypothalamic-Pituitary-Adrenal (HPA) Axis}

The glucocorticoid hypothesis has been repeatedly implicated in pathophysiological mechanisms of depression in both human and animal studies (de Kloet et al., 1998, 2005). Stress stimulates the HPA axis neuroendocrine pathway beginning with the release of corticotrophin-releasing hormone (CRH) from the hypothalamic paraventricular nucleus (PVN), causing adrenocorticotropic hormone (ACTH) secretion from the anterior pituitary and subsequent interaction at the adrenal medulla and lead to the release of glucocorticoid (GC) into circulation. The main GC in humans is cortisol, while in rodents, it is corticosterone. The stress response is turned off by GC feedback at GC receptors (GR) and mineralocorticoid receptors (MR) at the brain and pituitary sites. However, chronic stress- induced activation of the HPA axis impairs the negative regulatory mechanisms of these peripheral/central receptors (Pariante et al., 2001; Sapolsky et al., 2000). Subsequent hypercortisolemia, (measured in the plasma, urine, and cerebrospinal fluid (CSF) (Burke et al., 2005) is one of the most common clinical observations in depressed patients (de Kloet et al., 2005), resulting in systemic vascular and metabolic effects. GCs can directly and indirectly contribute to vasoconstrictive responses and endothelial dysfunction. GCs directly cause endothelial alterations that decrease $\mathrm{NO}$ bioavailability by limiting tetrahydrobiopterin $\left(\mathrm{BH}_{4}\right)$ activity and eNOS gene transcription as well as increasing the production of ROS. Further, proinflammatory cytokines disrupt GC receptor function on immune cells via activation of NF-kB, p38MAPK and the 5-STATS pathways, which inhibits expression of the active receptor (Roy and Campbell, 2013) and accelerates low-grade, chronic inflammation (Roy and Campbell, 2013; Rohleder, 2012). Concurrently, GC receptor function is reportedly reduced 
in lymphocytes of depressed patients (Rohleder, 2012), thus failing to attenuate immune responses (Roy and Campbell, 2013). In addition, permissive functions of GCs enhance the vascular vasoconstrictor responses of other stress-associated hormones, such as catacholamines and angiotensin II via upregulation of their receptor expression

in vascular smooth muscle cells (Ullian and Walsh, 1995). Together, these processes can damage vascular endothelium and impair endothelial dependent vasoreactivity. This is supported by studies that have found treatment with GC synthesis inhibitor improves endothelial dysfunction in depressed patients (Young and Ribiero, 2006).

\section{$\underline{\text { Inflammation }}$}

Some researchers have claimed that inflammatory events are the initial underlying cause of chronic stress/depression and CVD related factors (Manabe, 2011). Patients with depression exhibit elevated plasma levels of inflammatory markers, including prostaglandin $\mathrm{E}_{2}\left(\mathrm{PGE}_{2}\right), \mathrm{CRP}$, TNF- $\alpha$, IL-1 $\beta$, IL-2 and IL-6, in the peripheral plasma and CSF. TNF $\alpha$ and IL-6 impact both endothelial function and glucose metabolism, and have been linked to insulin resistance via autophosphorylation of insulin receptor substrate-1 (Hotamisligil et al., 1996). TNF $\alpha$ stimulates nuclear transcription factor-kappa B (NF-kB), a critical mediator of inflammatory responses, apoptosis, and expression of growth factors, proinflammatory cytokines and adhesion molecules, such as MCP-1, which is involved in macrophage recruitment (Knight and Imig, 2007). IL-6 is the primary regulator of C-reactive protein (CRP), and thus, increases CRP-mediated activity within the vasculature, resulting in inhibition of eNOS, elevated expression of adhesion molecules, and angiotensin-stimulated ROS production (Singer and Granger, 2007). These data demonstrate that one factor can contribute to vasculopathies via multiple mechanisms.

Animal models of chronic stress/depression have shown increased protein levels of TNF $\alpha, \mathrm{CRP}, \mathrm{MCP}-1$, and expression of intercellular adhesion molecule 1 (I-CAM1) (Lu et al., 2013). However, reports regarding inflammatory markers in chronic stress/depression and related pathology have proven to be largely inconsistent and difficult to replicate experimentally. This has been confirmed by previous studies indicating the multifaceted relationship and complex components of chronic stress/depression pathology. The relevance of individual vascular inflammatory markers and CV risk factors measured in vessels of UCMS mice was based on the specific disease conditions as well as the individual developmental route by which chronic stress influenced vascular pathologic events/outcomes (d'Audifffret et al., 2010). Division of the vascular outcomes into tertiles based on severity 
improved correlative values with specific risk biomarkers, but the variability in individual UCMS animals was still great. Data presented in Figure 3 shows the correlation between a given tertile of vascular impairment severity and specific CVD risk factors. These data demonstrate that aggregate measures of insulin resistance, inflammation, and hypertension are not robust predictors of vascular dysfunction, and unidentified mechanisms may be involved in heterogeneous outcomes between (d'Audifrett et al., 2010) individual inbred rodents. Human studies also reflect substantial heterogeneity in response to stress along with marked disparities in the pathophysiological outcome of depressive symptoms on comorbid diseases (Brosse et al., 2002).

Figure 3: Correlations between vascular reactivity and individual CVD risk factors in control and UCMS mice following separation of the lower bound of the methacholine concentration-response curves into tertiles based on the severity of impairment in dilator responses.

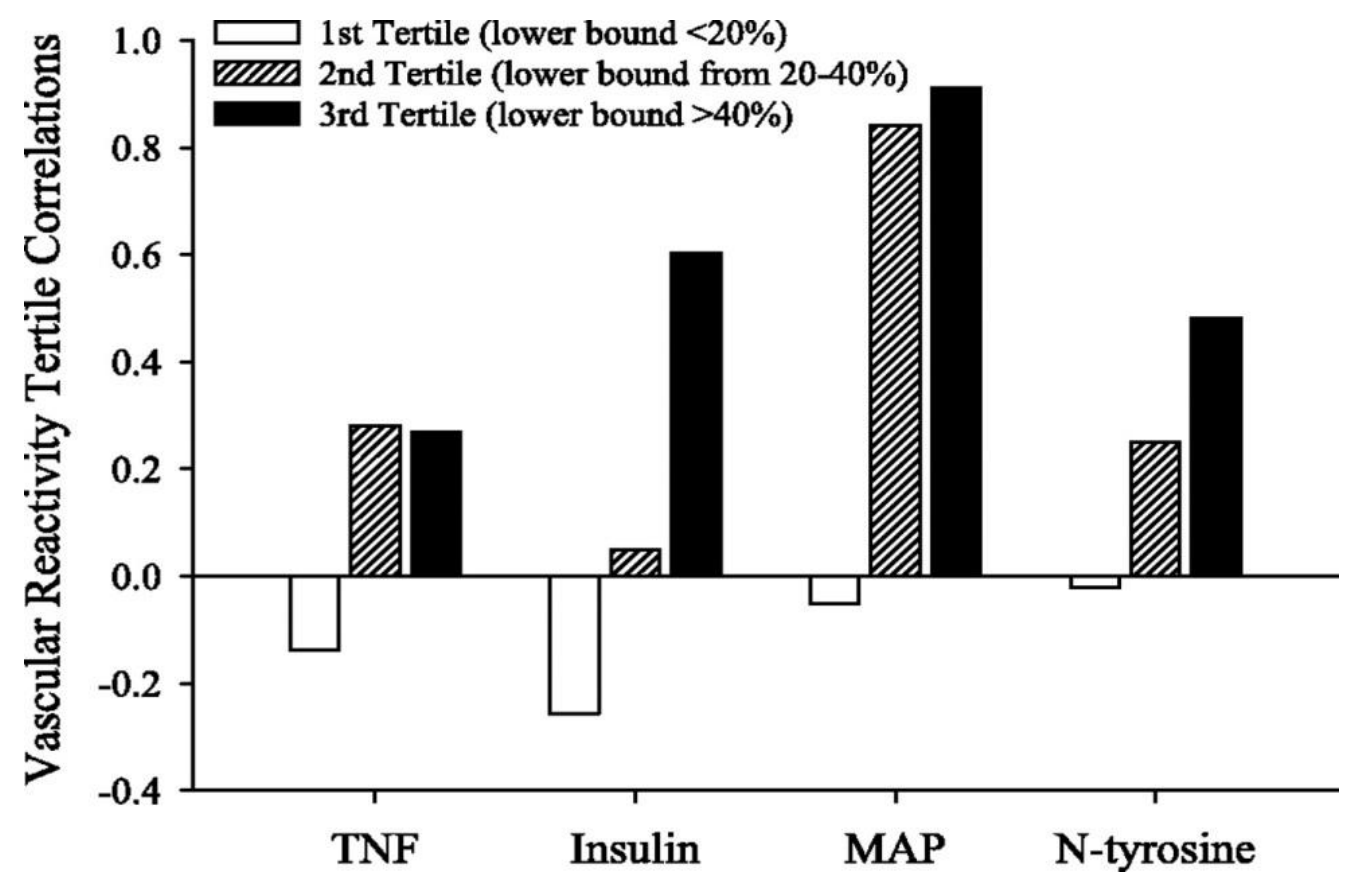




\section{Oxidative stress}

Reactive Oxygen Species (ROS) are short lived, highly reactive derivatives of oxygen metabolism involved with many normal biochemical processes in non-disease conditions. However, increased ROS production can overwhelm the endogenous antioxidant capacity of the system and cause deleterious damages that oxidize lipids, denature the function/structure of protein and DNA and activate redox-sensitive transcription factors and signal transduction pathways. In addition, ROS can interact with various cell receptors to down-regulate the production of antioxidants, such as superoxide dismutase (SOD), Glutathione, and catalase, further disrupting the oxidative/antioxidative balance in favor of unchecked ROS production. Primary sources of vascular oxidant stress are the NADPH oxidase family, xanthine/xanthine oxidase, uncoupled eNOS, cyclooxygenase, lipoxygenase, and cytochrome P450, as well as disrupted mitochondrial electron transport system (Figure 4).

Figure 4: Sources of ROS

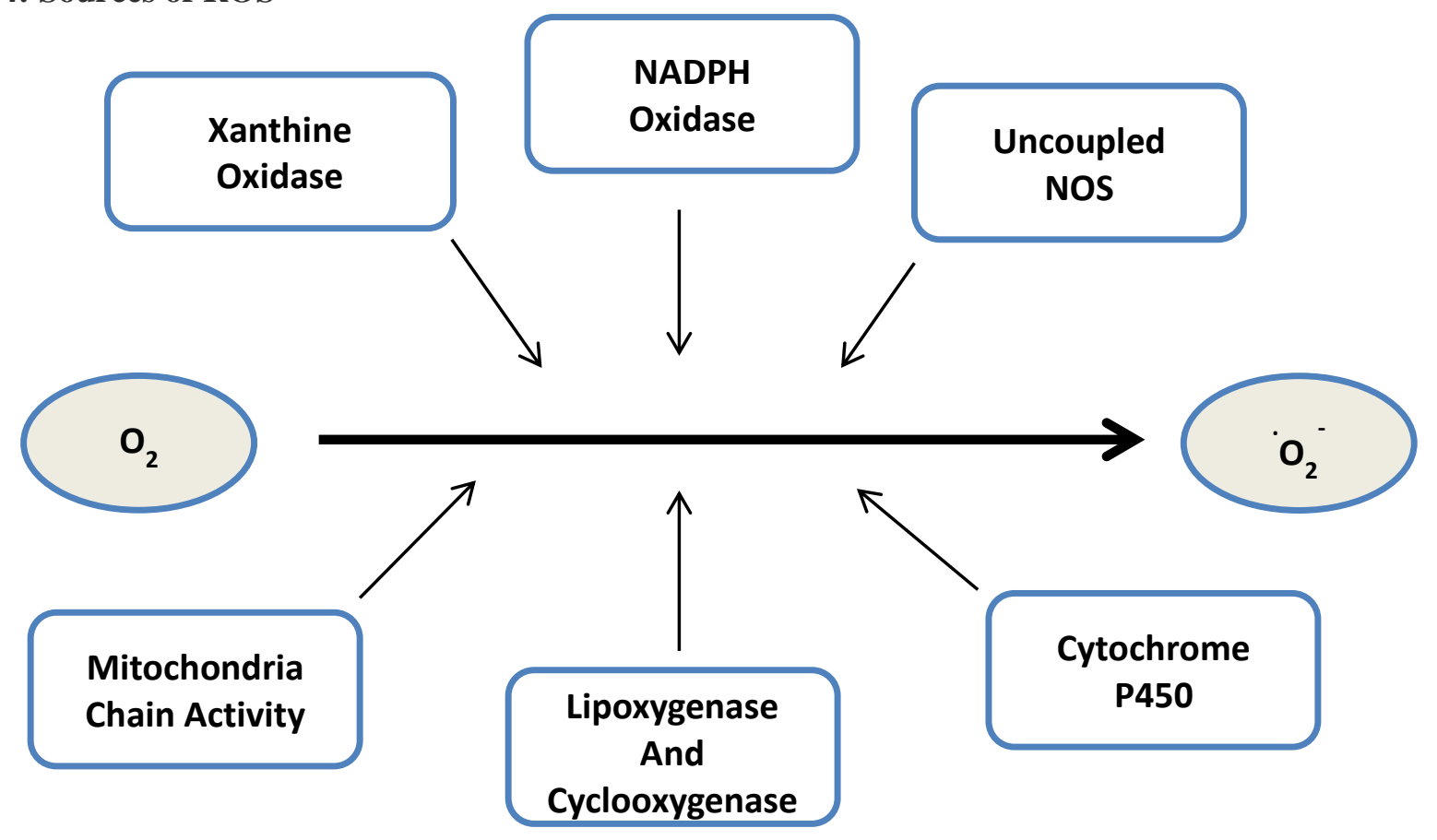


In pre-clinical animal models of hypertension and diabetes, oxidative stress and impaired endothelial function have been attributed to reduced bioavailability of NO and shown to increase production of superoxide anion, one of the most reactive oxygen radicals (Davis and Zou, 2005). These pathways are shown in Figure 5. Superoxide $\left(\cdot \mathrm{O}_{2}{ }^{-}\right)$can interact with $\mathrm{NO}$ to form the potent compound peroxynitrite $\left(\cdot \mathrm{ONOO}^{-}\right)$, which in turn readily induces uncoupling of eNOS, via oxidizing cofactor $\mathrm{BH}_{4}$, thus having a twofold impact: scavenging $\mathrm{NO}$ as well as inhibiting the activity of eNOS (Goodwill and Frisbee, 2012). Peroxynitrite also is involved in inactivating prostacyclin synthase (Davis and Zou, 2005). Oxidative stress is increased in UCMS animals and impaired vasodilator responses in the aortic rings and GAs was reversed with SOD mimetic treatment. However, treatment with a combination of TEMPOL and eNOS inhibitor abolished the effects of TEMPOL alone in UCMS male mice, suggesting that uncoupling of eNOS was contributing largely to endothelial dysfunction (Stanley et al., 2014). Further studies have demonstrated that increased superoxide production within the vasculature was associated with endothelial vasomotor dysfunction, which was reversed by the administration of agents capable of scavenging superoxide, such as vitamin C (Heitzer et al., 2001). These findings suggest that increased oxidative stress may be an important mechanism that impairs endothelial function in patients with atherosclerosis or cardiovascular risk factors, as well as in patients with chronic stress/depression.

Oxidative stress can alter the balance between circulating vasodilatory and vasoconstrictive factors, thereby changing a major regulatory component of vasoactivity and vascular tone (Goodwill et al., 2008). A wellestablished example is the role of arachidonic acid (AA) metabolism and COX pathway. The membrane-bound lipid, AA, is freed following cleavage by a phospholipase, enabling AA to diffuse into the cytoplasm of the cell where it can be metabolized by three different cytosolic enzymatic pathways: cyclooxygenase (COX), cytochrome P-450, and lipoxygenase. The COX pathway catalyzes the intermediate prostaglandin $\mathrm{H}_{2}$ into opposing vasoactive compounds, namely $\mathrm{PGI}_{2}$ via prostacyclin synthase, or $\mathrm{TXA}_{2}$ via thromboxane synthase. $\mathrm{PGI}_{2}$ increases vasodilation by a second messenger cyclic adenosine monophosphate (cAMP) signaling pathway and has similar, but less profound, effects within the vasculature compared to NO (Ledoux et al., 2006). Conversely, thromboxane mediates platelet activation, endothelial cell activation, vasoconstriction, and smooth muscle cell proliferation (Traupe et al., 2002). Recent reports demonstrated that production of $\mathrm{TxA}_{2}$ is increased while $\mathrm{PGI}_{2}$ is decreased in an animal 
model of MetS, significantly impacting vascular tone and severity of adrenergic constriction (Goodwill et al., 2008). Further, clinical studies have shown that patients with CVD have increased propensity to generate $\mathrm{TXA}_{2}$, more activate thromboxane receptors, and blunted platelet-mediated vasodilatation (Traupe et al., 2002). A shift in the production of COX metabolites favoring vasoconstriction has been implicated in disease states such as hypertension, CAD, diabetes, and depression.

Figure 5: Pathways of ROS Production in the Vascular Endothelium

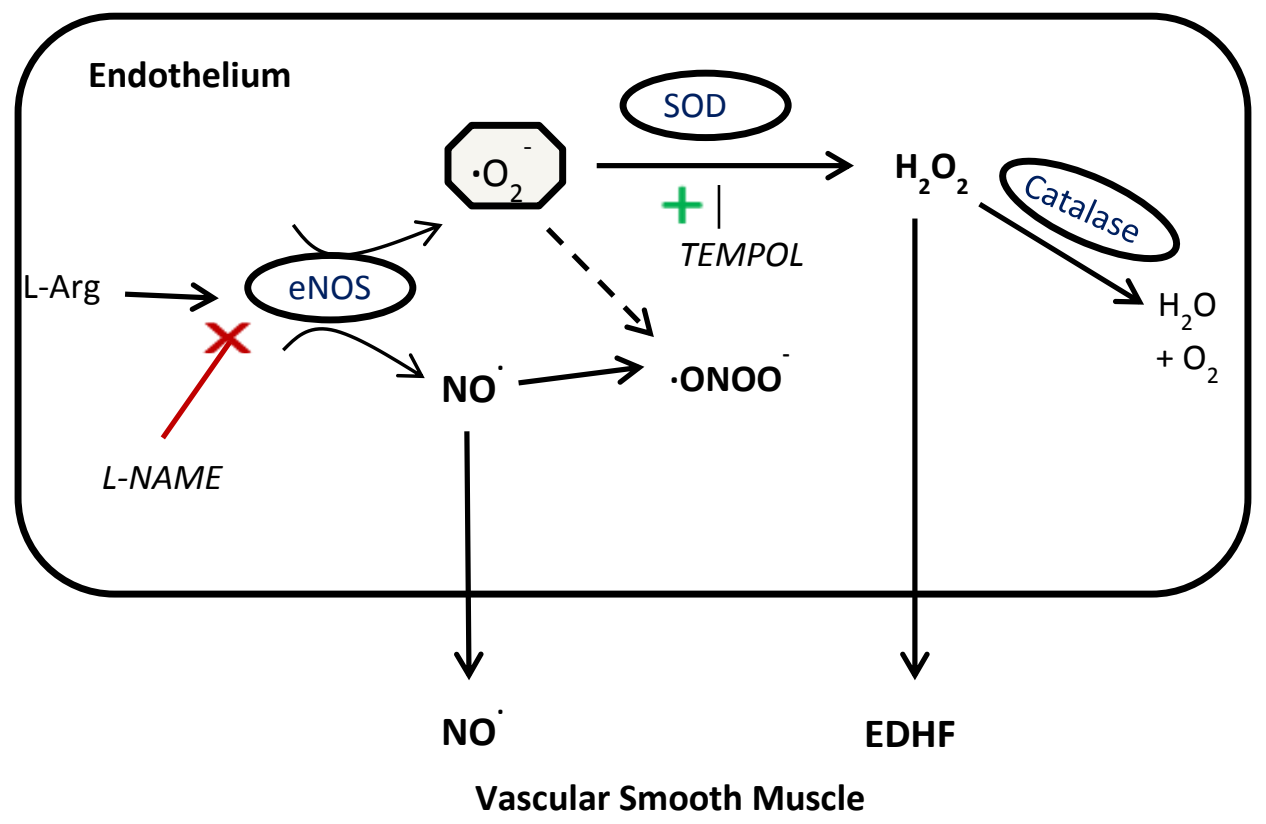

Endothelium produces a less well-characterized compound known as endothelial dependent hyperpolarizing factor (EDHF) that promotes vascular smooth muscle relaxation and vasodilation. The exact contributing factors making up EDHF metabolites are unclear, but hydrogen peroxide $\left(\mathrm{H}_{2} \mathrm{O}_{2}\right)$ has been indicated. $\mathrm{H}_{2} \mathrm{O}_{2}$ is the product of SOD mediated reaction of water and superoxide. In a previous study, UCMS induced impairments to vasodilator responses to methacholine were more severely blunted following inhibition of $\mathrm{H}_{2} \mathrm{O}_{2}$ with catalase in aortic rings compared to controls (d'Audiffret et al., 2010), suggesting the promotion of a compensatory mechanism when NO levels are reduced. This is shown in Figures 6A and 6B. 
Figure 6: Dilator responses of aortic rings to increasing concentrations of methacholine in control and UCMS mice.
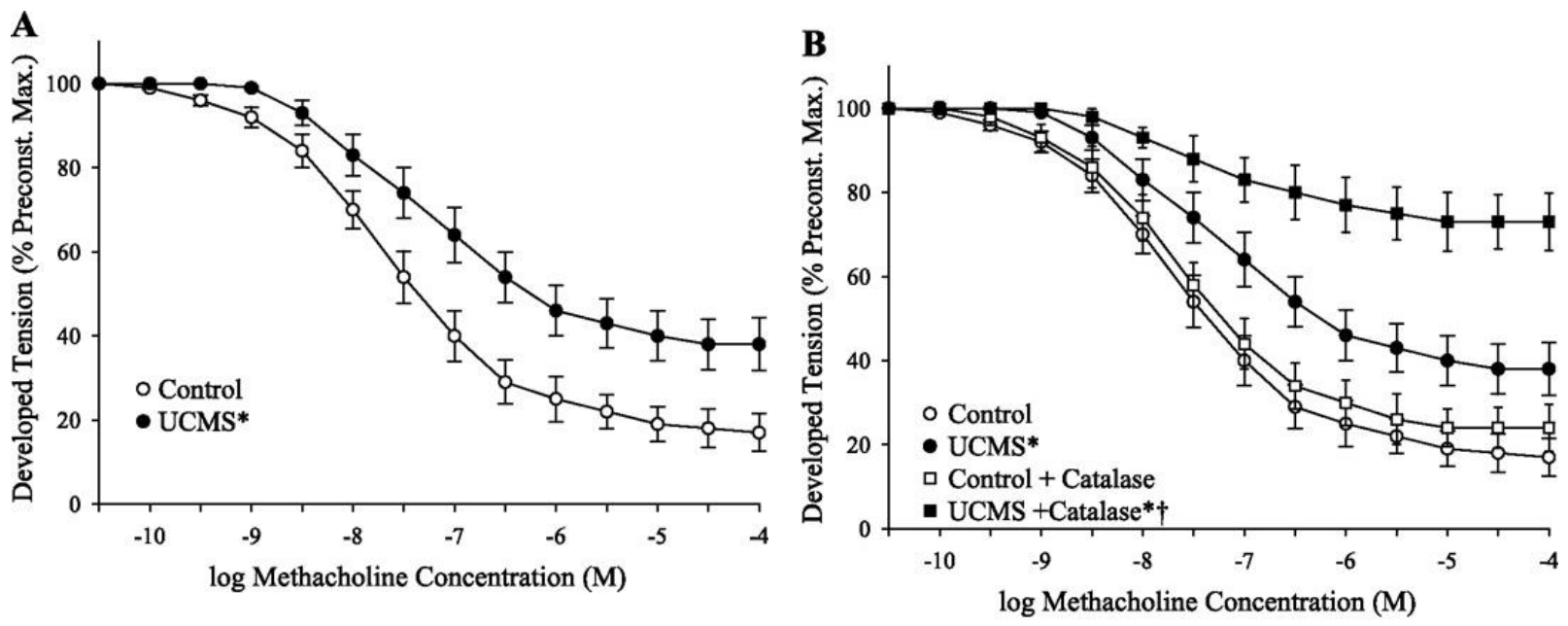

$* P<0.05$ vs. responses in untreated vascular rings from control mice; $\uparrow P<0.05$ vs. responses in untreated vascular rings from UCMS mice; $n=8$ for control; $n=8-9$ for UCMS.

There have been reports showing differences in the induction of the oxidative response between the sexes. Specifically, a relationship has been reported between estrogen and general cardiovascular protection and antioxidant defense that reduce reactive oxygen species and thereby increase NO bioactivity (Kamper et al., 2009; MacRitchie et al., 1997). The antioxidant effects of estrogen have been reported to occur through genomic as well nongenomic alterations (Gomez-Zubeldia et al., 2000; Borras et al., 2005) while testosterone has been shown to decrease antioxidant (superoxide dismutase, glutathione peroxidase) activity (Chainy et al., 1997).

\section{Clinical Significance}

Depression imparts a serious deleterious effect on chronic medical conditions, with potentially adverse influences on self-care (e.g., adherence to diet, exercise, cessation of smoking, use of medication), disease control, symptom burden, development of medical complications, quality of life, and the cost of healthcare. Similarly, chronic medical conditions, especially one as tightly linked to stress-related events as CVD, are affectively stressful for patients and may influence pathophysiologic mechanisms as well as mood related to depression. Depression imparts a serious deleterious effect on chronic medical conditions, with potentially adverse influences on self-care (e.g., adherence to diet, exercise, tobacco use, medical compliance), disease control, symptom 
burden, development of medical complications, quality of life, and the cost of healthcare. Thus, understanding the bidirectional relation between depression and comorbid CVD is an essential goal in order to ensure optimal health care.

Furthermore, the marked sex disparity in depression risk and CVD development warrants further investigation to improve clinical healthcare; however, the precise sex-specific mechanisms regulating cardiovascular physiology and neurological function are largely unclear. An emergent area of research has focused on identifying functional pathways and mechanisms of sex hormones on processes of stress reactivity with the ultimate goal of understanding the divergent pathophysiologies of depression between males and females. Basic sex differences in homeostasis and autonomic signaling begin early in development and continue throughout adulthood, and resulting hormonal and neuroendocrine differences profoundly impact not only normal physiology, but also the pathways that link the stress response with susceptibilities to depression and comorbid CVD. Interrogation of the sex-specific mechanisms defining this link may lead to improvements in disease progression and prognosis for both men and women and facilitate development of more effective therapeutics and interventions that target sexspecific pathophysiological mechanisms of CVD development. 


\section{REFERENCES}

1. World Health Organization. Preventing Chronic Diseases: A Vital Investment. http://www.who.int/chp/chronic_disease_report/full_report.pdf 2005.

2. Alberti KG, Eckel RH, Grundy SM, et al. Harmonizing the metabolic syndrome: a joint interim statement of the International Diabetes Federation Task Force on Epidemiology and Prevention; National Heart, Lung, and Blood Institute; American Heart Association; World Heart Federation; International Atherosclerosis Society; and International Association for the Study of Obesity Circulation 2009; 120 1640-1645.

3. Arnett DK, Goodman RA, Halperin JL, Anderson JL, Parekh AK, et al. AHA/ACC/HHS Strategies to Enhance Application of Clinical Practice Guidelines in Patients With Cardiovascular Disease and Comorbid Conditions Circulation. 2014; 130:1662-1667.

4. Go AS, Mozaffarian D, Roger VL, et al. Heart disease and stroke statistics-2014 update: a report from the American Heart Association. Circulation. 2014; 129:e28-292.

5. Chantler PD, Frisbee JC. Arterial Function in Cardio-Metabolic Diseases: From the Microcirculation to the Large Conduits Prog Cardiovasc Dis. 2015; 57(5):489-96.

6. Ward BW, Schiller JS. Prevalence of multiple chronic conditions among US adults: estimates from the National Health Interview Survey, 2010. Prev Chronic Dis. 2013; 10:E65.

7. Isingrini E, Surget A, Belzung C, Freslon JL, Frisbee J, O'Donnell J, Camus V, d'Audiffret A. Altered aortic vascular reactivity in the unpredictable chronic mild stress model of depression in mice: UCMS causes relaxation impairment to ACh. Physiol Behav. 2011; 103(5):540-6.

8. Grover-Páez F, Zavalza-Gómez AB. Endothelial dysfunction and cardiovascular risk factors. Diabetes Res Clin Pract. 2009; 84(1):1-10.

9. Frisbee JC, Hollander JM, Brock RW, Yu HG, Boegehold MA Integration of skeletal muscle resistance arteriolar reactivity for perfusion responses in the metabolic syndrome. Am J Physiol Regul Integr Comp Physiol. 2009; 296(6):R1771-82.

10. Penninx BW, Beekman AT, Honig A, Deeg DJ, Schoevers RA, van Eijk JT, van Tilburg W. Depression and cardiac mortality: results from a community-based longitudinal study. Arch Gen Psych. 2001; 58(3):221-7. 
11. Rutledge T, Reis SE, Olson MB, Owens J, Kelsey SF, Pepine CJ, Mankad S, Rogers WJ, Merz CN, Sopko G, Cornell CE, Sharaf B, Matthews KA, Vaccarino V. Depression symptom severity and reported treatment history in the prediction of cardiac risk in women with suspected myocardial ischemia: The NHLBIsponsored WISE study.Arch Gen Psychiatry. 2006; 63(8):874-80.

12. Pizzi C, Manzoli L, Mancini S, Bedetti G, Fontana F, Costa GM. Autonomic nervous system, inflammation and preclinical carotid atherosclerosis in depressed subjects with coronary risk factors. Atherosclerosis. 2010; 212(1):292-8

13. Barbero C, Gilchrist S, Schooley MW, Chriqui JF, Luke DA, Eyler AA. Appraising the Evidence for Public Health Policy Components Using the Quality and Impact of Component Evidence Assessment.Glob Heart $2015 ; 10(1): 3-11$.

14. Kannan H, Thompson S, Bolge SC. Economic and humanistic outcomes associated with comorbid type-2 diabetes, high cholesterol, and hypertension among individuals who are overweight or obese. J Occup Environ Med. 2008; 50:542-549.

15. Mahoney EM, Wang K, Keo HH et al., Vascular hospitalization rates and costs in patients with peripheral artery disease in the United States. Circ Cardiovasc Qual Outcomes 2010; 3(6):642-51.

16. Ouriel K. Peripheral arterial disease. Lancet 2001; 358(9289):1257-64.

17. Ford ES, Mokdad AH. Epidemiology of obesity in the Western hemisphere. J Clin Endocrinol Metab 93, Suppl 1: S1-S8, 2008.

18. Cannon CP. Cardiovascular disease and modifiable cardiometabolic risk factors. Clin Cornerstone. 2007; $8: 11-28$.

19. Steinberg BA, Bhatt DL, Mehta S, Poole-Wilson PA, O'Hagan P, Montalescot G, Ballantyne CM, Cannon CP. Nine-year trends in achievement of risk factor goals in the US and European outpatients with cardiovascular disease. Am Heart J 2008; 156:719-727.

20. Goodwill AG, Frisbee JC. Oxidant stress and skeletal muscle microvasculopathy in the metabolic syndrome Vascul Pharmacol 2012; 57(5-6):150-9. 
21. Zhou Z, de Beer VJ, de Wijs-Meijler D, Bender SB, Hoekstra M, Laughlin MH, Duncker DJ, Merkus D. Pulmonary vasoconstrictor influence of endothelin in exercising swine depends critically on phosphodiesterase 5 activity. Am J Physiol Lung Cell Mol Physiol. 2014; 306(5):L442-52.

22. Esler M, Rumantir M, Wiesner G, Kaye D, Hastings J, Lambert G. Sympathetic nervous system and insulin resistance: from obesity to diabetes, Am. J. Hypertens. 2001; (14)304S-309S.

23. Frisbee JC. Vascular adrenergic tone and structural narrowing constrain reactive hyperemia in skeletal muscle of obese Zucker rats, Am. J. Physiol. Heart Circ. Physiol. 2006; 290:H2066-H2074.

24. Schreihofer AM, Hair CD, Stepp DW. Reduced plasma volume and mesenteric vascular reactivity in obese Zucker rats, Am. J. Physiol. Regul. Integr. Comp. Physiol. 2005; 288 R253-R261.

25. Frisbee JC, Goodwill AG, Butcher JT, Olfert IM. Divergence between arterial perfusion and fatigue resistance in skeletal muscle in the metabolic syndrome. Exp Physiol 2011; 96(3):369-83.

26. Goodwill AG, Frisbee SJ, Stapleton PA, James ME, Frisbee JC. Impact of chronic anticholesterol therapy on development of microvascular rarefaction in the metabolic syndrome. Microcirculation 2009; 16(8):667-84.

27. Schiffrin EL, Touyz RM. From bedside to bench to bedside: role of renin-angiotensin-aldosterone system in remodeling of resistance arteries in hypertension Am J Physiol Heart Circ Physiol 2004; 287: H435-H446

28. Chan SY, Mancini GB, Kuramoto L, Schulzer M, Frohlich J, Ignaszewski A. The prognostic importance of endothelial dysfunction and carotid atheroma burden in patients with coronary artery disease. J Am Coll Cardiol. 2003; 42(6):1037-43.

29. Perticone F, Ceravolo R, Pujia A, Ventura G, Iacopino S, Scozzafava A, Ferraro A, Chello M, Mastroroberto P, Verdecchia P, Schillaci G. Prognostic significance of endothelial dysfunction in hypertensive patients. Circulation. 2001; 104(2):191-6.

30. Neunteufl T, Heher S, Katzenschlager R, Wölfl G, Kostner K, Maurer G, Weidinger F. Late prognostic value of flow-mediated dilation in the brachial artery of patients with chest pain. Am J Cardiol. 2000; 86(2):207-10.

31. Gokce N, Keaney JF, Hunter LM, Watkins MT, Nedeljkovic ZS, Menzoian JO, Vita JA. Predictive value of noninvasively determined endothelial dysfunction for long-term cardiovascular events in patients with peripheral vascular disease. J Am Coll Cardiol. 2003; 41(10):1769-75. 
32. Sherwood A, Hinderliter AL, Watkins LL, Waugh RA, Blumenthal JA. Impaired endothelial function in coronary heart disease patients with depressive symptomatology. J Am Coll Cardiol. 2005; 46(4):656-9.

33. Zou, M.H., C. Shi, R.A. Cohen. Oxidation of the zinc-thiolate complex and uncoupling of endothelial nitric oxide synthase by peroxynitrite. J. Clin. Invest. 2002; 109(6):817-826.

34. Bouzinova EV, Møller-Nielsen N, Boedtkjer DB, Broegger T, Wiborg O, Aalkjaer C, Matchkov VV. Chronic mild stress-induced depression-like symptoms in rats and abnormalities in catecholamine uptake in small arteries. Psychosom Med. 2012; 74(3):278-87B.

35. Davis M, Zou H. CD40 ligand-dependent tyrosine nitration of prostacyclin synthase in vivo Circulation, 2005; $112(14) 2184-2192$.

36. Stanley SC, Brooks SD, Butcher JT, d'Audiffret AC, Frisbee SJ, Frisbee JC. Protective effect of sex on chronic stress- and depressive behavior-induced vascular dysfunction in BALB/cJ mice. J Appl Physiol (1985). 2014; 117(9):959-70.

37. Goodwill AG, James ME, Frisbee JC. Increased vascular thromboxane generation impairs dilation of skeletal muscle arterioles of obese Zucker rats with reduced oxygen tension Am. J. Physiol. Heart Circ. Physiol., 2008; 295(4):H1522-H1528.

38. Hotamisligil GS, Peraldi P, Budavari A, Ellis R, White MF, Spiegelman BM. IRS-1-mediated inhibition of insulin receptor tyrosine kinase activity in TNF-alpha- and obesity-induced insulin resistance Science, 1996; 271 (5249):665-668.

39. Knight SF, Imig JD. Obesity, insulin resistance, and renal function. Microcirculation, 2007; 14(4-5-: 349-362

40. Singer G, Granger DN. Inflammatory responses underlying the microvascular dysfunction associated with obesity and insulin resistance Microcirculation, 2007; 14(4-5): 375-387.

41. Lu XT, Liu YF, Zhao L, Li WJ, Yang RX, Yan FF, Zhao YX, Jiang F Chronic psychological stress induces vascular inflammation in rabbits. Stress. 2013; 16(1):87-98.

42. Heyes MP, Saito K, Crowley JS, Davis LE, Demitrack M A, Der M, et al. Quinolinic acid and kynurenine pathway metabolism in inflammatory and non-inflammatory neurological disease. Brain 1992;115(Pt. 5): $1249-1273$. 
43. Francis GS, Cohn JN, Johnson G, Rector TS, Goldman S, Simon A. Plasma norepinephrine, plasma renin activity and congestive heart failure. Relations to survival and the effects of therapy in V-HeFT II. The VHeFT VA cooperative studies group. Circulation 1996; 87(Suppl. 6).

44. Ganguly PK, Dhalla KS, Innes IR, Beamish RE, Dhalla NS. Altered norepinephrine turnover and metabolism in diabetic cardiomyopathy. Circ. Res. 1986; 59:684-693.

45. Wilson CB, McLaughlin LD2, Ebenezer PJ, Nair AR, Dange R1, Harre JG, Shaak TL, Diamond DM, Francis J. Differential effects of sertraline in a predator exposure animal model of post-traumatic stress disorder. Front Behav Neurosci. 2014; 8:256.

46. Hart EC, Charkoudian N, Miller VM. Sex, hormones and neuroeffector mechanisms. Acta Physiol; 2011; 203, 155-165.

47. Yang H, Drummer TD, Carter JR. Sex differences in sympathetic neural and limb vascular reactivity to mental stress in humans. Am J Physiol Heart Circ Physiol 2013; 304: H436-H443, 2013.

48. de Kloet ER, Vreugdenhil E, Oitzl MS, Joels M. Brain corticosteroid receptor balance in health and disease. Endocr Rev 1998; 19(3):269-301.

49. de Kloet ER, Joels M, Holsboer F. Stress and the brain: from adaptation to disease. Nat Rev Neurosci 2005; 6:463-75.

50. Pariante CM, Miller AH. Glucocorticoid receptors in major depression: relevance to pathophysiology and treatment. Biol Psychiatry 2001; 49:391-404.

51. Sapolsky RM. Glucocorticoids and hippocampal atrophy in neuropsychiatric disorders. Arch Gen Psychiatry 2000; 57:925-35.

52. Kamper EF, Chatzigeorgiou A, Tsimpoukidi O, Kamper M, Dalla C, Pitychoutis PM, Papadopoulou-Daifoti Z. Sex differences in oxidant/antioxidant balance under a chronic mild stress regime Physiol Behav. 2009; 98(1-2):215-22.

53. Moller-Leimkuhler A.M. Gender differences in cardiovascular disease and comorbid depression Dialog. Clin. Neurosci. 2007; 9:71-83. 
54. Heitzer T, Schlinzig T, Krohn K, Meinertz T, Munzel T. Endothelial dysfunction, oxidative stress, and risk of cardiovascular events in patients with coronary artery disease. Circulation 2001; 104:2673-2678.

55. Naik JS, Xiang L, Hester RL. Enhanced role for RhoA-associated kinase in adrenergic-mediated vasoconstriction in gracilis arteries from obese Zucker rats. Am J Physiol Regul Integr Comp Physiol 2006; 290: R154-R161.

56. Willner P. Validity, reliability and utility of the chronic mild stress model of depression: a 10-year review and evaluation. Psychopharmacology (Berl). 1997; 134: 319-329.

57. Yalcin I, Belzung C, Surget A. Mouse strain differences in the unpredictable chronic mild stress: a fourantidepressant survey. Behav Brain Res. 2008; 193: 140-143.

58. Mineur YS, Prasol DJ, Belzung C, Crusio WE. Agonistic behavior and unpredictable chronic mild stress in mice. Behav Genet. 2003; 33: 513-519.

59. Chrapko WE, Jurasz P, Radomski MW, Lara N, Archer SL, Le Mellédo JM. Decreased platelet nitric oxide synthase activity and plasma nitric oxide metabolites in major depressive disorder. Biol Psychiatry. 2004; 56(2):129-34.

60. Rajagopalan S, Brook R, Rubenfire M, Pitt E, Young E, Pitt B. Abnormal brachial artery flow-mediated vasodilation in young adults with major depression. Am J Cardiol. 2001; 88(2):196-8, A7.

61. Dimopoulos N, Piperi C, Salonicioti A, Mitsonis C, Liappas I, Lea RW, Kalofoutis A. Elevation of plasma concentration of adhesion molecules in late-life depression. Int J Geriatr Psychiatry. 2006; 21(10):965-71.

62. Kessler RC, Berglund P, Demler O, Jin R, Koretz D, Merikangas KR, Rush AJ, Walters EE, Wang PS. National Comorbidity Survey Replication. The epidemiology of major depressive disorder: results from the National Comorbidity Survey Replication (NCS-R). JAMA 2003; 289:3095-3105.

63. Kessler RC, McGonagle KA, Swartz M, Blazer DG, Nelson CB. Sex and depression in the National Comorbidity Survey. I: lifetime prevalence, chronicity and recurrence. J Affect Disord. 1993; 29:85-96.

64. Lloyd-Jones D, Adams RJ, Brown TM, Carnethon M, Dai S, DeSimone G, Ferguson TB, Ford E, Furie K, Gillespie C, Go A, Greenlund K, Haase N, Hailpern S, et al. American Heart Association Statistics 
Committee and Stroke Statistics. Executive summary: heart disease and stroke statistics-2010 update: a report from the American Heart Association. Circulation 121: 948-954, 2010.

65. Pizzi C, Manzoli L, Mancini S, Bedetti G, Fontana F, Costa GM. Autonomic nervous system, inflammation and preclinical carotid atherosclerosis in depressed subjects with coronary risk factors. Atherosclerosis 2010; 212: 292-298.

66. Koledova VV, Khalil RA. Sex hormone replacement therapy and modulation of vascular function in cardiovascular disease. Expert Rev Cardiovasc Ther 2007; 5:777-789.

67. Duckles SP, Miller VM. Hormonal modulation of endothelial NO production. Pflugers Arch - Eur J Physiol 2010; 459:841-851.

68. Daniel JM, Hulst JL, Lee CD. Role of hippocampal M2 muscarinic receptors in the estrogen-induced enhancement of working memory. Neuroscience 2005; 132: 57-64.

69. MacRitchie AN, Jun SS, Chen Z, German Z, Yuhanna IS, Sherman TS, Shaul PW. Estrogen upregulates endothelial nitric oxide synthase gene expression in fetal pulmonary artery endothelium. Circ Res 1997; 81: $355-362$.

70. Borras C, Gambini J, Gomez-Cabrera MC, Sastre J, Pallardo FV, Mann GE, et al. 17beta-oestradiol upregulates longevity-related, antioxidant enzyme expression via the ERK1 and ERK2[MAPK]/NFkappaB cascade. Aging Cell 2005; 4:113-8.

71. Gomez-Zubeldia MA, Hernandez R, Viguera J, Arbues JJ, Aparicio A, Millan JC. Effect of bilateral ovariectomy and ovarian steroid hormones on the antioxidant systems and plasma malondialdehyde levels in Wistar rats. Endocr Res 2000; 26:97-107.

72. Chainy GB, Samantaray S, Samanta L. Testosterone-induced changes in testicular antioxidant system. Andrologia 1997; 29:343-9.

73. Puzserova A, Torok J, Sotnikova R, Zemancikova A, Bernatova I. Reactivity of the mesenteric bed arteries of normotensive rats exposed to chronic social stress. Gen Physiol Biophys. 2012; 31(3):279-90.

74. Manabe I. Chronic inflammation links cardiovascular, metabolic and renal diseases. Circ. J. 2011; 75(12):2739-2748. 
75. Young EA, Ribiero SC. Sex differences in the ACTH response to $24 \mathrm{H}$ metyrapone in depression. Brain Res 2006; 1126: 148-155.

76. Vaccarino V, Parsons L, Every NR., Barron HV, Krumholz HM. Sex-based differences in early mortality after myocardial infarction. N Engl J Med. 1999; 341:217-225.

77. Bello N, Mosca L. Epidemiology of coronary heart disease in women. Progr Cardiovasc Dis. 2004; 46:287295.

78. Eastwood JA, Doering LV. Gender differences in coronary artery disease. J Cardiovasc Nurs. 2005; 20:430351.

79. Jackson W F. Potassium channels in the peripheral cicrocirculation. Microcirculation. 2005; 12:113-127.

80. Ledoux J, Werner ME, Brayden JE, Nelson M T. Calcium-activated potassium channels and the regulation of vascular tone. Physiology 2006; 21: 69-78.

81. Traupe T, Lang M, Goettsch W, Munter K, Morawietz H, Vetter W, Barton M. Obesity increases prostanoidmediated vasoconstriction and vascular thromboxane receptor gene expression. J Hypertens 2002; 20: 22392245.

82. Van Itallie C. Thyroid hormone and dexamethasone increase the levels of a messenger ribonucleic acid for a mitochondrially encoded subunit but not for a nuclear-encoded subunit of cytochrome c oxidase. Endocrinology. 1990; 127:55-62.

83. Strekalova T, Couch Y, Kholod,N et al. Update in the methodology of the chronic stress paradigm: internal control mattersBehav. Brain Funct., 2011; 7, 9.

84. Penninx B, Beekman A, Honig A, Deeg D, Schoevers R, van Eijk JT, van Tilburg W. Depression and cardiac mortality: results from a community-based longitudinal study. Arch Gen Psychiatry. 2001;58(3):221-7

85. Kim EY, Miklowitz DJ, Biuckians A, Mullen K. Life stress and the course of early-onset bipolar disorder J. Affect. Disord. 2007; 99 (1-3).

86. Mansura RB, Brietzkeb E, McIntyrea RS. Is there a "metabolic-mood syndrome"? A review of the relationship between obesity and mood disorders. Neuroscience \& Biobeh Reviews. 2015; 52:89-104. 
87. Cosentino F, Eto M, De Paolis P, Van Der Loo B, Bachschmid M, Ullrich V, Kouroedov A, Delli Gatti C, Joch H, Volpe M, Luscher TF. High glucose causes upregulation of cyclooxygenase-2 and alters prostanoid profile in human endothelial cells: role of protein kinase $\mathrm{C}$ and reactive oxygen species. Circulation. 2003; 107:1017-1023.

88. Kelly AS, Wetzsteon RJ, Kaiser DR, Steinberger J, Bank AJ, Dengel DR. Inflammation, insulin, and endothelial function in overweight children and adolescents: the role of exercise. J Pediatr 2004; 145:731736.

89. Goldstein JM, Jerram M, Abbs B, Whitfield-Gabrieli S, Makris N. Sex differences in stress response circuitry activation dependent on female hormonal cycle. J Neurosci 2012; 30:431-438.

90. Tobet SA, Handa RJ, Goldstein JM. Sex-dependent pathophysiology as predictors of comorbidity of major depressive disorder and cardiovascular disease. Pflugers Arch 2013; 465:585-594.

91. Antonijevic IA. Depressive disorders -- is it time to endorse different pathophysiologies? Psychoneuroendocrinology, 2006; 31(1):1-15.

92. Ullian ME, Walsh LG. Corticosterone metabolism and effects on angiotensin II receptors in vascular smooth muscle. Circ. Res. 1995; 77(4):702-709.

93. Brosse AL, Sheets ES, Let HS, Blumenthal JA. Exercise and the Treatment of Clinical Depression in Adults Recent Findings and Future Directions Sports Med. 2002; 32 (12):741-760. 


\section{Chapter 1:}

PROTECTIVE EFFECT OF SEX ON CHRONIC STRESS- AND DEPRESSIVE BEHAVIORINDUCED VASCULAR DYSFUNCTION IN BALB/cJ MICE

Shyla C. Stanley ${ }^{1,2}$, Steven D. Brooks ${ }^{1,2}$, Joshua T. Butcher ${ }^{1,2}$, Alexandre C. d'Audiffret ${ }^{1,3}$, Stephanie J. Frisbee $^{1,4}$, and Jefferson C. Frisbee ${ }^{1,2}$

Center for Cardiovascular and Respiratory Sciences ${ }^{1}$, Departments of Physiology and Pharmacology ${ }^{2}$, Vascular and Endovascular Surgery ${ }^{2}$ and Health Policy, Leadership and Management ${ }^{4}$; West Virginia University Health Sciences Center, Morgantown, WV 26506

Running Head: Stress, vascular dysfunction and sex

Send Correspondence to:

Jefferson C. Frisbee, Ph.D.

Center for Cardiovascular and Respiratory Sciences

Department of Physiology and Pharmacology

Robert C. Byrd Health Sciences Center

PO Box 9105

West Virginia University School of Medicine

Morgantown, WV 26505

Phone: (304) 293-6527

Fax: (304) 293-5513

Email: jefrisbee@hsc.wvu.edu 


\section{ABSTRACT}

The presence of chronic, unresolvable stresses leads to negative health outcomes, including development of clinical depression/depressive disorders, with outcome severity being correlated to depressive symptom severity. One of the major outcomes associated with chronic stress and depression is the development of cardiovascular disease (CVD) and an elevated CVD risk profile. However, in epidemiological research, sex disparities are evident, with pre-menopausal females suffering from depressive symptoms more acutely than males, but also demonstrating a relative protection from the onset of CVD. Given this, we investigated the differential effect of sex on conduit artery and resistance arteriolar function in male and female mice following eight weeks of an unpredictable chronic mild stress (UCMS) protocol. In males, plasma cortisol and depressive symptom severity (e.g., coat status, anhedonia, delayed grooming) were elevated by UCMS. Endotheliumdependent dilation to methacholine/acetylcholine was impaired in conduit arteries and skeletal muscle arterioles, suggesting a severe loss of NO bioavailability and increased production of $\mathrm{TxA}_{2}$ versus $\mathrm{PGI}_{2}$ associated with elevated reactive oxygen species and an increased level of systemic inflammation. Endothelium-independent dilation was intact. In females, depressive symptoms and plasma cortisol increases were more severe than in males, although alterations to vascular reactivity were blunted, including the effects of elevated ROS and inflammation on dilator responses. These results suggest that, in comparison to males, female rats are more susceptible to chronic stress in terms of the severity of depressive behaviors, but that the subsequent development of vasculopathy is blunted owing to an improved ability to tolerate elevated ROS and systemic inflammatory stress.

Key Words: $\quad$ vasodilation, endothelium-derived factors, peripheral vascular disease, nitric oxide, models of chronic stress, clinical depression 


\section{INTRODUCTION}

During the last decade, extensive evidence from epidemiologic and clinical studies has identified a complex relationship between depressive disorders and cardiovascular disease (CVD) outcomes (34, 41, 44, 45, 58). Depression is a powerful risk factor for development of CVD, and can be a predictor of cardiovascular pathologies, including myocardial infarction, coronary artery disease, and cardiomyopathies, regardless of prior history of overt CVD $(41,44)$.

A consistent body of evidence indicates that chronic stress, a major contributor to depressive illnesses, may be a potent pathogenic factor linking depression and CVD, in part due to the stress-induced activation of the sympathetic-adrenalmedullary and hypothalamic pituitary adrenal (HPA) axes (1, 2, 4, 7, 9, 47). Dysregulation of these two essential systems has been linked to depression and the development of several CVD risk factors, including sex-specific pathophysiological factors associated with autonomic nervous and immune dysfunction, and alterations to vascular reactivity and endothelial function $(20,22,49$. 56). Compared to age-matched male counterparts, pre-menopausal women are at lower risk of developing CVD $(29,30)$, but concurrently are twice as likely to suffer from depressive disorders $(28,35)$. This trend diminishes at menopause and risk of CVD and neuropsychological disorders in women rapidly increases $(32,35)$. Current literature supports an association between estrogen and the progression of depressive symptoms and comorbid diseases $(17,35,55)$, but also a relationship between estrogen and general $\mathrm{CV}$ protection and antioxidant defense $(6,27,37)$.

We $(12,14,26.42)$, and others $(3,5,27,39)$ have previously reported that the impact of chronic stress and depression on aortic ring reactivity is mediated through altered endothelial dysfunction, characterized by an impaired dilator metabolite bioavailability. A comparable outcome, including poor endothelium-dependent reactivity $(14,25,26,36)$, nitric oxide bioavailability $(42,48)$, as well as a pro-inflammatory $(24,36,39)$ and prothrombotic (43) environment, has also been observed in depressed human patients, and represents and an independent predictor of risk for the development of $\operatorname{CVD}(18,48)$. As circulating levels of estrogen also exert a positive impact on processes associated with vascular function $(6,27,35,37)$, the impact of sex on CVD under conditions of chronic stress and depression is an area that warrants investigation. 
The unpredictable chronic mild stress (UCMS) procedure is a widely used animal model of induced depressive symptoms and has been well-established in behavioral studies as an extremely relevant rodent model of human depression based upon its ability to reproduce clinical symptoms of depression, including anhedonia and learned helplessness $(25,46,59)$. Studies utilizing the UCMS protocol have reported increased behavioral vulnerability to UCMS in female rodents $(23,32,45)$ associated with increased cortisol levels $(15,16)$, altered estrous cycle $(15,16,23,31)$, and decreased serotonergic and dopaminergic turnover ratios in brain regions related to stress and depression $(16,33)$. However, compared to male counterparts, UCMS female rodents show greater antioxidant defense and increased NO bioavailability, helping to maintain normal endothelial function and enhanced vascular anti-inflammatory and antioxidant capacities $(27,50)$.

The purpose of this study was to determine the differential susceptibility to depressive symptom development following UCMS between male and female mice, and to elucidate sex-specific mechanisms underlying vascular/endothelial dysfunction in both conduit vascular rings and in pressurized resistance arterioles. This study tested the hypothesis that 8 weeks of UCMS will result in a more severe development of depressive symptoms in female mice (versus males), but that vascular function will be preserved to a superior extent than in males. Of special note, this study utilized a protocol wherein the estrous cycle of the female mice was not matched. Rather female mice were randomized throughout their cycle to get a better assessment of a true sex difference rather than one that reflects cyclic variations in hormonal status.

\section{MATERIALS AND METHODS}

Animals: Male and female BALB/cJ mice (Jackson) were fed standard chow and drinking water ad libitum and were housed in the animal care facility at the WVU HSC. All protocols received prior IACUC approval. At nine weeks of age, mice from each sex were divided into two groups, control and UCMS (below). After eight weeks duration under either condition, mice were anesthetized with injections of sodium pentobarbital (50 mg•kg-1 i.p.) and a carotid artery was cannulated for determination of arterial pressure. Venous blood aliquots were collected for biochemical evaluation of plasma biomarkers of treatment outcomes and health status of the mice. 
Unpredictable Chronic Mild Stress Protocol: All mice were doubly housed, with the control group in a separate quiet room in close proximity to the room used for UCMS treatments. Alternatively, in the UCMS group, mice were randomly exposed to the following stressors on multiple occasions throughout each 24 hour period:

1. Damp bedding - $10 \mathrm{oz}$. of water was added to each standard cage for the next 3 hours

2. Water - all bedding was removed and $\sim 0.5$ inches of water was added to empty cage for the next 3 hours. Water temperature was $\sim 30^{\circ} \mathrm{C}$ and the room temperature was $\sim 24^{\circ} \mathrm{C}$

3. Each cage was tilted to 45 degrees with or without bedding for 3 hours

4. Social stress - each mouse was switched into a cage of a neighboring mouse for 3 hours

5. No bedding lasting for 3 hours or, on two occasions each week, overnight.

6. Succession of light/dark cycles, lasting 30 minutes throughout a 24 hour period

7. Exposure to predator smells (e.g., cat fur) and/or sounds (e.g., cat growling) for 8 hours

After eight weeks, all mice were subjected to a series of behavioral tests to evaluate the outcomes of the UCMS procedures.

Coat Status: This evaluation was done throughout the duration of the UCMS protocol. The total cumulative score was computed by giving an individual score of 0 (clean) or 1 (dirty) to eight body parts (head, neck, dorsal coat, ventral coat, tail, forelimb, hind-limb, and genital region).

Splash Test: This test was used to evaluate acute grooming behavior, defined as cleaning of the fur by licking or scratching. A $10 \%$ sucrose solution was sprayed on the dorsal coat of each mouse and grooming activity was recorded for five minutes. The viscosity of the sucrose solution will dirty the coat and induce grooming behavior, with depressive symptoms characterized by an increased latency (idle time between spray and initiation of grooming) and decreased frequency (number of times grooming a particular body part).

Tail Suspension Test: Mice subjected to the short term, inescapable stress of tail suspension, will develop an immobile posture, with longer periods of immobility in mice exhibiting a depressed behavior. Mice were completely suspended by the tail on a horizontal bar $35 \mathrm{~cm}$ from the base platform using adhesive tape such that contact with the laboratory benchtop was not possible. The latency to the first bout of immobility and the duration of total immobility were recorded for six minutes. 
Measurements of Vascular Reactivity (Skeletal Muscle Resistance Arterioles): From each anesthetized mouse, the intramuscular continuation of the gracilis artery was removed and cannulated, as described previously (8). These first order arterioles were extended to their approximate in situ length and were equilibrated at $80 \%$ of mean arterial pressure in order to approximate in vivo intralumenal pressure. Following equilibration, the reactivity of isolated, pressurized arterioles was assessed in response to increasing concentrations of phenylephrine $\left(10^{-10} \mathrm{M}-10^{-7} \mathrm{M}\right)$ to assess adrenergic constrictor responses and increasing concentrations of acetylcholine $\left(10^{-10} \mathrm{M}-\right.$ $\left.10^{-6} \mathrm{M}\right)$ and sodium nitroprusside $\left(10^{-10} \mathrm{M}-10^{-6} \mathrm{M}\right)$ to assess dilator reactivity. Vascular responses to stimuli were also determined following treatment with L-NAME $\left(10^{-4} \mathrm{M}\right)$, indomethacin $\left(10^{-5} \mathrm{M}\right)$ and TEMPOL $\left(10^{-4} \mathrm{M}\right)$ for 45 60 minutes, to assess the roles of nitric oxide, cyclooxygenase and reactive oxidant stress, respectively, in modulating reactivity.

Measurements of Vascular Reactivity (Conduit Arteries): Following removal of the resistance arteriole in each mouse, the thoracic aorta was removed, rinsed in physiological salt solution, cleared of surrounding tissue, and cut in 2-3 mm ring segments. Each ring was mounted in a myobath chamber between a fixed point and a force transducer (World Precision Instruments), and set to $0.5 \mathrm{~g}$ tension for 45 minutes to equilibrate. The organ baths contained physiological salt solution at $37^{\circ} \mathrm{C}$, and aerated with $95 \% \mathrm{O}_{2}$ and $5 \% \mathrm{CO}_{2}$. Rings were pre-conditioned by treatment with $10^{-7} \mathrm{M}$ phenylephrine for 5 minutes, at which time $10^{-5} \mathrm{M}$ methacholine was added to the bath to assess endothelial integrity. Any ring that failed to demonstrate both a brisk constrictor response to phenylephrine and viable endothelial function was discarded. Subsequently, rings were treated with increasing concentrations of phenylephrine $\left(10^{-10} \mathrm{M}-10^{-4} \mathrm{M}\right)$ to assess constrictor reactivity. For the assessment of dilator reactivity, rings were pre-treated with $10^{-6} \mathrm{M}$ phenylephrine and exposed to increasing concentrations of methacholine $\left(10^{-10} \mathrm{M}-10^{-4} \mathrm{M}\right)$ and sodium nitroprusside $\left(10^{-10} \mathrm{M}-10^{-4} \mathrm{M}\right)$. To assess the roles of nitric oxide, cyclooxygenase and reactive oxidant stress in modulating vascular responses to the agonist treatments, concentration-response curves were also conducted following pre-treatment of the rings for 45-60 minutes with L-NAME $\left(10^{-4} \mathrm{M}\right)$, indomethacin $\left(10^{-5} \mathrm{M}\right)$ and TEMPOL $\left(10^{-4} \mathrm{M}\right)$, respectively.

Measurement of Vasoactive Metabolite Bioavailability: From mice within each group, the remaining sections of the thoracic and abdominal aorta that were not used for measurements of reactivity were used to assess vascular 
$\mathrm{NO}, \mathrm{PGI}_{2}$ (from levels of 6-keto-PGF ${ }_{1 \alpha}$ ) and $\mathrm{TxA}_{2}$ (from levels of 11-dehydro- $\mathrm{TxB}_{2}$ ) bioavailability using amperometric sensors (World Precision Instruments) and a commercially available kits (Cayman), respectively. Briefly, aortae were isolated, cleaned, sectioned into $\sim 1 \mathrm{~mm}$ lengths and placed within a chamber filled with physiological salt solution equilibrated with $21 \% \mathrm{O}_{2}, 5 \% \mathrm{CO}_{2}$, balance $\mathrm{N}_{2}$. Within this chamber, a nitric oxide sensor (ISO-NOPF 100) was inserted and a baseline current was obtained. Subsequently, methacholine $\left(10^{-8}, 10^{-}\right.$ ${ }^{6}$, and $10^{-4} \mathrm{M}$ ) was added to the chamber and the changes in current were determined. To verify that responses represented NO release, these procedures were repeated following addition of L-NAME $\left(10^{-4} \mathrm{M}\right)$ to the chamber. In response to challenge with $10^{-4} \mathrm{M}$ methacholine, an aliquot of PSS was removed from the chamber and used for determination of $\mathrm{PGI}_{2}$ and $\mathrm{TxA}_{2}$ production.

Biochemical Analyses: In all samples, fasting blood glucose ( 8 hour) was determined using a commercially available glucometer (Freestyle). Chronic oxidant stress was assessed through determination of plasma nitrotyrosine and plasma cortisol was determined using standard kits (Cayman). Finally, using a multiplexed procedure, plasma insulin and biomarkers of inflammation were assessed using commercially available kits (Millipore).

Data and Statistical Analyses: Mechanical responses following challenge with methacholine or phenylephrine were fit with the three-parameter logistic equation:

$$
y=\min +\left[\frac{\max -\min }{1+10^{\log E D_{50}-x}}\right]
$$

where $y$ represents the isometric tension, "min" and "max" represent the lower (minimum) and upper (maximum) bounds, respectively, of the change in tone with agonist concentration, $x$ is the logarithm of the agonist concentration and $\log E D_{50}$ represents the logarithm of the agonist concentration $(x)$ where the response $(y)$ is halfway between the bounds. The use of the three-parameter logistic equation is appropriate for the analysis of sigmoidal concentration-response relationships, as it simultaneously provides estimates of the curve maximum (upper bound), minimum (lower bound) and the dose at which the dependent variable reaches $50 \%$ of maximum $\left(\mathrm{ED}_{50}\right)$. In the case of a dilator response where the initial condition is set to $100 \%$ (the upper bound), the asymptotic minimum (the lower bound from the equation) is reflective of the degree of dilator reactivity for that vessel. If the vessel becomes "more reactive", the lower bound will decrease toward reduced levels, while if the vessel becomes less 
reactive, the lower bound will increase toward higher values. This situation is reversed for constrictor responses, where the vessel starts at the lower bound, and then goes through a range of increased tensions to reach an upper bound/asymptotic maximum. For the presentation of results, we have focused on the changes in the upper or lower bounds, as we did not determine a consistent or significant change to the ED50 values between relevant experiment groups.

All data are presented as mean \pm SEM. Significant differences between groups were determined using analysis of variance (ANOVA). In all cases, Student-Newman-Keuls post hoc test was used when appropriate and $\mathrm{p}<0.05$ was taken to reflect statistical significance.

\section{RESULTS}

Table 1 presents data describing the characteristics of mouse groups in the present study. At the time of final use, plasma insulin and nitrotyrosine were significantly greater in UCMS mice than in controls. No consistent and significant differences were determined between body mass, mean arterial pressure and blood glucose between conditions or sex. Plasma levels of cortisol were significantly increased with UCMS in both sexes as compared to controls, and levels in UCMS-females were significantly greater than that in UCMS-males.

The impact of the UCMS protocol on depressive behaviors in mice is summarized in Figure 1. Throughout 8 weeks of UCMS, the coat status in mice undergoing the stress protocol was consistently poorer compared to that in control animals (Panel A), although the severity of this degradation in coat status was significantly worse in UCMSfemales. In response to the sucrose spray, control mice demonstrated both a more rapid (Panel B) and a more frequent (Panel C) grooming response as compared to that exhibited by mice of either sex following the UCMS protocol. However, the latency of the grooming response was greater in UCMS-females as compared to that in UCMS-males. Panel D presents the total period of immobility in response to tail suspension, where control mice demonstrated a significantly shorter period of immobility as compared to that in UCMS mice of either sex. Consistent with the previous measurements, UCMS-females demonstrated a longer period of immobility as compared to UCMS-males. 
The constrictor responses of aortic rings from control and UCMS-mice in response to increasing concentrations of phenylephrine are summarized in Figure 2. As compared to responses in aortic rings from control mice, rings from UCMS-males exhibited a similar constrictor response to the adrenergic agonist (Panel A). In contrast, the constrictor response of aortic rings from UCMS-females was not different from that in controls. Following pre-treatment of vessels from all groups with L-NAME, responses of aortic rings from the control and UCMS groups remained similar in response to increasing concentrations of phenylephrine (Panel B). Pre-treatment of aortic rings with indomethacin did not significantly alter responses to phenylephrine from that determined under untreated conditions in any group (data not shown).

A summary of the methacholine-induced dilator responses of aortic rings from control and UCMS-mice is presented in Figure 3. In comparison to responses from control mice, the dilation to increasing concentrations of methacholine was significantly reduced in both male and female UCMS mice, although the severity of this blunted response was greater in the aortic rings from UCMS-males (Panel A). In aortic rings from control animals, pretreatment with either L-NAME or indomethacin resulted in a significant inhibition of methacholine-induced dilator reactivity, while pre-treatment with both nearly abolished all responses to the agonist (Panels B and C). In aortic rings from UCMS-males, pre-treatment with L-NAME did not further impact the reduced dilator responses to methacholine, although treatment with indomethacin reduced dilator responses from the untreated condition (Panel D). In conduit artery segments from UCMS-female rats, treatment with L-NAME reduced methacholine-induced dilation, while responses following treatment with indomethacin were less striking (Panel E). In all cases, combined treatment with both L-NAME and indomethacin nearly abolished the reactivity of aortic rings to increasing concentrations of methacholine.

Figure 4 summarizes the responses of aortic rings from control and UCMS-mice to increasing concentrations of sodium nitroprusside. In all cases, application of the nitric oxide donor resulted in a significant dilator response that was not different between control and UCMS-mice of either sex.

The production of dilator metabolites in response to methacholine challenge is presented in Figure 5. Vascular production of nitric oxide following application of methacholine was significantly reduced in conduit arteries of UCMS-mice of either sex, although the severity of this reduction was greater in UCMS-males (Panel A). 
A similar pattern was determined for the vascular production of $\mathrm{PGI}_{2}$, with the methacholine-induced production of this metabolite, estimated from 6-keto-PGF ${ }_{1 \alpha}$, being reduced in UCMS mice of both sexes (male>female) as compared to responses in arteries from control mice (Panel B). Vascular production of $\mathrm{TxA}_{2}(\mathrm{Panel} \mathrm{C})$ was low and similar in male and female mice under control conditions. In response to the UCMS protocol, the vascular production of $\mathrm{TxA}_{2}$ was significantly elevated in both sexes, but was significantly increased in UCMS males as compared to UCMS females.

The impact of pre-treatment of aortic rings with the antioxidant TEMPOL on vascular production of nitric oxide following methacholine challenge is presented in Figure 6. While bioavailability of nitric oxide was attenuated in vessels from both male and female mice following UCMS treatment (males>females), pre-treatment of vessels with TEMPOL significantly increased the bioavailability of nitric oxide in vessels from UCMS-males. While a trend for this was evident in UCMS-females, this effect was not as striking, likely owing to the more modest reductions in NO bioavailability as a result of the UCMS protocol itself in these mice. Treatment of vessels with L-NAME abolished methacholine-induced NO bioavailability in all conditions.

The effects of pre-treatment of aortic rings from UCMS-mice with TEMPOL on dilator responses are summarized in Figure 7. In aortic rings from both UCMS-male (Panel A) and UCMS-female (Panel B) mice, pretreatment with TEMPOL significantly increased dilator responses to methacholine from the untreated responses; subsequent treatment of vessels with L-NAME or indomethacin significantly reduced dilation from this improved level of reactivity.

The constrictor responses of isolated skeletal muscle (gracilis) arterioles in response to increasing concentrations of phenylephrine are summarized in Figure 8. In arterioles from male rats, imposition of the UCMS protocol resulted in a significant increase in the vasoconstrictor responses to phenylephrine as compared to responses in vessels from control animals (Panel A). Pre-treatment of vessels with L-NAME abolished all differences in phenylephrine-induced constriction between sexes and treatment condition (Panel B).

Figure 9 presents the dilator responses of gracilis muscle arterioles from control and UCMS-treated mice in response to increasing concentrations of acetylcholine. In males, UCMS treatment blunted dilator responses to acetylcholine from levels in controls; pretreatment of vessels from UCMS-males with L-NAME had no effect on 
reactivity, while pretreatment with indomethacin further blunted this impaired response (Panel A). However, treatment of arterioles from UCMS-males with TEMPOL was very effective at restoring dilator reactivity to acetylcholine (Panel B). The dilator response in UCMS-females in response to acetylcholine was reduced as compared to that from controls, and pre-treatment with either L-NAME or indomethacin caused a further reduction in reactivity (Panel C). Similar to that in UCMS-males, treatment of vessels from UCMS-females with TEMPOL restored dilator responses to increasing concentrations of acetylcholine (Panel D).

The correlation between UCMS-induced changes in systemic inflammation and vascular dysfunction is summarized in Figure 10 for TNF- $\alpha$ (Panel A) and MCP-1 (Panel B). The degree of vascular dysfunction, estimated from the lower bound of the methacholine concentration-response relationship for aortic rings (determined from the logistic equation described above), demonstrated a positive relationship with the degree of systemic inflammation as a result of the UCMS protocol. However, when split into sexes, a more severe relationship was evident in males, where similar levels of either TNF- $\alpha$ (Panel A) or MCP-1 (Panel B) was associated with a greater degree of vascular dysfunction (i.e., a higher lower bound) in males than in females. This was evident under both control and UCMS conditions. Interestingly, within either sex, imposition of the UCMS protocol did not appear to change the inherent relationship between markers of inflammation and vascular dysfunction. Rather, the relationship between the parameters remained similar, but was shifted to a higher severity of dysfunction.

\section{DISCUSSION}

With an escalating body of clinical and epidemiological evidence demonstrating that sex differences influence the link between chronic stress, the onset of depressive symptoms and cardiovascular disease risk and outcomes, validated preclinical models offer a valuable translational tool for investigating the underlying mechanisms of disease and offer an efficacious method for studying novel interventional therapies and treatment options. This study utilized the UCMS protocol, a validated model of chronic stress-induced depressive symptoms in rodents, to interrogate sex-specific mechanisms of vascular dysfunction in UCMS mice.

The results of this study provide evidence that male and female mice experience graded outcomes related to the severity of depressive symptom development and an almost paradoxically gradation of the impairment to 
vascular reactivity after 8 weeks of the UCMS protocol. Increased behavioral susceptibility to stress conditions was observed in female mice versus male counterparts, as evidenced by worsened coat grooming score, increased immobility in the tail suspension test (behavioral despair), and elevated plasma levels of corticosterone. Estrogen levels are known to amplify the magnitude of the stress response at the HPA axis $(4,23,57,60,61)$, offering a potential explanation for the increased corticosterone and behavioral symptom severity observed in the UCMS female mice. UCMS-induced impairments to vascular reactivity and function were observed for both sexes, demonstrated by blunting of dilator responses to methacholine/acetylcholine in isolated aortic rings and in pressurized resistance arterioles, respectively. However, these physiological impairments were also sex-specific, as female UCMS mice developed greater elevations to biomarkers of oxidative stress and inflammation, yet demonstrated a more modest impairment to vascular reactivity, despite the more severe deterioration in the behavioral measures.

Building on our previous report that vasodilator reactivity is blunted in aortic rings of UCMS male mice $(14,26)$, our new findings suggest that the impaired dilator responses in UCMS mice of both sexes are due to endothelial dysfunction, as dilations to exogenous NO were similar across all groups, as were constrictor responses to phenylephrine, suggesting that an 8-week UCMS protocol is not sufficient to significantly alter vascular wall mechanics or vascular smooth muscle function. Vasodilator responses were further blunted in aortic rings and gracilis arterioles of UCMS females following pre-treatment with L-NAME; however this treatment had minimal effects on vascular reactivity in UCMS males, likely owing to the pre-existing severe attenuation of vascular NO bioavailability determined in UCMS males. Instead, the blunted dilator responses in vessels from UCMS males were further impaired as a result of COX inhibition with INDO, suggesting that a dependence on dilator influence of arachidonic acid metabolites (likely $\mathrm{PGI}_{2}$ ) helps to maintain vascular reactivity in the compromised state. However, vascular $\mathrm{PGI}_{2}$ production alone did not correlate strongly across all of the observed mechanical responses, suggesting a potential contributing role for other COX metabolites. Indeed, proinflammatory, oxidative stress conditions induced by chronic stress can alter the balance between constricting and dilating metabolites, specifically shifting arachidonic acid metabolism by $\mathrm{COX}$ in favor of increased production of vasoconstricting metabolites (e.g., thromboxane, $\mathrm{TxA}_{2}$ ) that can compete against the effect of stimulus induced 
vasodilation by $\mathrm{NO}, \mathrm{PGI}_{2}$ or other dilator metabolites $(14,26)$. Our results suggest that an elevation in stimulusinduced vascular $\mathrm{TxA}_{2}$ generation may contribute to the integrated vasoreactivity in this model. Given the integral role of inflammation and oxidant stress that has been demonstrated with chronic stress and depressive symptoms, and the impact of chronic elevations in vascular $\mathrm{TxA}_{2}$ production will have on negative cardiovascular outcomes in terms of perfusion control and the anti-thrombotic functions (among others) of the endothelium, the impact of chronic inhibition of these pathways on both the behavioral and vascular outcomes in UCMS-induced depressive symptoms clearly represents important areas for future interrogation.

While the results of this study found only very limited evidence of an increased vascular reactivity to adrenergic constriction in UCMS males, these results largely appear to be a reflection of the degree to which endothelial function was impaired as a result of the UCMS protocol. However, the vasoconstrictor response of all animal groups to increasing concentrations of phenylephrine converged at a very similar levels following inhibition of NOS activity via pre-treatment with L-NAME, suggesting that the role endogenous NO bioavailability plays in moderating adrenergic constriction under normal conditions remains largely intact in females despite UCMS, but is diminished in males following UCMS protocols.

The relationship between systemic inflammation induced by UCMS and the severity of vascular dysfunction observed in males and females were investigated to determine sex differences in the effects of chronic inflammation on vascular reactivity. Circulating plasma levels of pro-inflammatory markers MCP-1 and TNF- $\alpha$ were associated with vascular dysfunction for all UCMS animals, but a greater degree of vascular dysfunction was observed in UCMS-males than in UCMS-females, despite the observation that the magnitude of the inflammatory markers was somewhat higher in female mice. When taken together with the higher plasma levels of ROS in female UCMS mice versus males as well, these data suggest males experience a substantially greater endothelial susceptibility to UCMS-induced dysfunction, raising the possibility of a protective mechanism for female sex hormones that effectively reduces impairment of endothelium-dependent vasodilation during exposure to chronic stress.

There is extensive evidence for the vasoprotective actions of estrogen against oxidative and inflammatory stressors. Estrogen increases nitric oxide synthase activity and promotes NO bioavailability from the vascular 
endothelium $(15,27,60)$; it is also linked to increased responsiveness to $\beta_{2}$-adrenergic-mediated vasodilation (19, $21,38)$, partially through a NO-related mechanism $(19,51)$. In addition to supportive effects on NO, estrogen may also influence production of $\mathrm{PGI}_{2}$ via up-regulation of the $\mathrm{COX}$ pathway, specifically $\mathrm{COX}-1$ and prostacyclin synthase in some vascular beds $(40,51,53,60)$. While less is known regarding androgens, it has been reported that testosterone supplementation impairs vascular reactivity and antioxidant capacity (10) in females by mitigating vascular sensitivity to NO $(11,52)$. Females may therefore have a more robust and protected endothelium-dependent vasodilator capacity than males due to higher levels of circulating estrogen and significantly less testosterone, thus mitigating the inflammatory and oxidative effects of chronic stress on vascular reactivity $(13,54,60)$.

That being stated, the purpose of the study was not to test the effects of cyclic variation in estrogen levels on vascular function, rather it was to determine the potential effect of a true difference in sex. As such, the female mice used in this study were randomized within their estrous cycle. Given the robustness of the differences in outcomes (both behavioral and vascular) as a result of the UCMS protocol, the fact that these mice were not studied at the same points in their estrous cycle has significant implications. Most critically, these results suggest that the protective effect associated with the female sex may not be critically dependent on the specific time within the estrous cycle or on specific hormonal profiles at that time, but that it may be much more stable and afford a degree of protection that is relatively insensitive to the day-to-day fluctuations in hormonal profiles. This is especially compelling as it suggests that the potentially beneficial and protective mechanisms that are associated with the female sex remain in place throughout the estrous cycle and may not fluctuate along the same temporal pattern as the hormonal levels. The molecular basis for this temporal pattern, as well as the alternate hypothesis that the lower levels of androgen hormones is actually the basis for the protection in this model of chronic stress and depressive symptoms and poor cardiovascular outcomes, will require future targeted investigation.

Summary and Conclusions: In response to imposition of chronic unresolvable stresses onto male and female mice, the results of the present study indicate that the behavioral impairments, elevations in plasma cortisol, and plasma markers of oxidant stress and inflammation demonstrated by female mice are significantly greater than those demonstrated by male mice. However, despite these elevated responses, the developed vasculopathy in both the 
conduit and resistance vasculature of female mice is less severe than that for males. Much of this blunted impairment appears to be a function of a superior maintenance of endothelial function, primarily via more normal levels of NO bioavailability and a more normal balance between the production of $\mathrm{PGI}_{2}$ and $\mathrm{TxA}_{2}$ to help maintain dilator reactivity. As female rats were randomized with respect to their time in estrous cycle, these data appear to reflect a longer, more temporally stable, protective effective of "being female" rather than one that reflects the more labile temporal changes in hormonal levels. In addition, the correlational analyses between inflammatory biomarkers and vascular dysfunction suggest that there are fundamental differences between male and female sex with regard to these associations. However, imposition of the UCMS protocol does not appear to cause the differences in the sex-based protections to diminish. Rather, UCMS appears to right-shift these associations along the same sex-specific lines to a higher degree of vascular dysfunction. Future research into the specific nature of the temporal nature of these protective effects and how they can be exploited therapeutically to minimize the devastating effects of depressive symptoms on cardiovascular health outcomes represents a key area for future investigation.

\section{ACKNOWLEDGEMENTS}

The authors gratefully acknowledge the Office of Laboratory Animal Research at West Virginia University for their expertise and assistance during the performance of these procedures. Additionally, we also acknowledge the support provided through Center for Cardiovascular and Respiratory Sciences at the West Virginia University Health Sciences Center.

\section{SOURCES OF RESEARCH FUNDING}

This study was supported by the American Heart Association (IRG 14330015, PRE 16850005, EIA 0740129N), and the National Institutes of Health (RR 2865AR; P20 RR 016477). 


\section{REFERENCES}

1. Antonijevic, I. A. (2006). Depressive disorders -- is it time to endorse different pathophysiologies? Psychoneuroendocrinology, 31(1), 1-15. doi: 10.1016/j.psyneuen.2005.04.004

2. Barden, N. (2004). Implication of the hypothalamic-pituitary-adrenal axis in the physiopathology of depression. J Psychiatry Neurosci, 29(3), 185-193.

3. Bayramgurler, D., Karson, A., Yazir, Y., Celikyurt, I. K., Kurnaz, S., \& Utkan, T. (2013). The effect of etanercept on aortic nitric oxide-dependent vasorelaxation in an unpredictable chronic, mild stress model of depression in rats. Eur J Pharmacol, 710(1-3), 67-72. doi: 10.1016/j.ejphar.2013.04.007

4. Beck, K. D., \& Luine, V. N. (2002). Sex differences in behavioral and neurochemical profiles after chronic stress: role of housing conditions. Physiol Behav, 75(5), 661-673.

5. Bilici, M., Efe, H., Koroglu, M. A., Uydu, H. A., Bekaroglu, M., \& Deger, O. (2001). Antioxidative enzyme activities and lipid peroxidation in major depression: alterations by antidepressant treatments. J Affect Disord, 64(1), 43-51.

6. Borras, C., Gambini, J., Gomez-Cabrera, M. C., Sastre, J., Pallardo, F. V., Mann, G. E., \& Vina, J. (2005). 17beta-oestradiol up-regulates longevity-related, antioxidant enzyme expression via the ERK1 and ERK2[MAPK]/NFkappaB cascade. Aging Cell, 4(3), 113-118. doi: 10.1111/j.1474-9726.2005.00151.x

7. Bowman, R. E., Beck, K. D., \& Luine, V. N. (2003). Chronic stress effects on memory: sex differences in performance and monoaminergic activity. Horm Behav, 43(1), 48-59.

8. Butcher, J. T., Goodwill, A. G., \& Frisbee, J. C. (2012). The ex vivo isolated skeletal microvessel preparation for investigation of vascular reactivity. J Vis $\operatorname{Exp}(62)$. doi: 10.3791/3674

9. Campbell, T., Lin, S., DeVries, C., \& Lambert, K. (2003). Coping strategies in male and female rats exposed to multiple stressors. Physiol Behav, 78(3), 495-504.

10. Chainy, G. B., Samantaray, S., \& Samanta, L. (1997). Testosterone-induced changes in testicular antioxidant system. Andrologia, 29(6), 343-349. 
11. Chen, M. J., Yang, W. S., Yang, J. H., Chen, C. L., Ho, H. N., \& Yang, Y. S. (2007). Relationship between androgen levels and blood pressure in young women with polycystic ovary syndrome. Hypertension, 49(6), 1442-1447. doi: 10.1161/HYPERTENSIONAHA.106.083972

12. Chrapko, W. E., Jurasz, P., Radomski, M. W., Lara, N., Archer, S. L., \& Le Melledo, J. M. (2004). Decreased platelet nitric oxide synthase activity and plasma nitric oxide metabolites in major depressive disorder. Biol Psychiatry, 56(2), 129-134. doi: 10.1016/j.biopsych.2004.03.003

13. Csiszar, A., Wang, M., Lakatta, E. G., \& Ungvari, Z. (2008). Inflammation and endothelial dysfunction during aging: role of NF-kappaB. J Appl Physiol (1985), 105(4), 1333-1341. doi: 10.1152/japplphysiol.90470.2008

14. d'Audiffret, A. C., Frisbee, S. J., Stapleton, P. A., Goodwill, A. G., Isingrini, E., \& Frisbee, J. C. (2010). Depressive behavior and vascular dysfunction: a link between clinical depression and vascular disease? $J$ Appl Physiol (1985), 108(5), 1041-1051. doi: 10.1152/japplphysiol.01440.2009

15. Dalla, C., Antoniou, K., Drossopoulou, G., Xagoraris, M., Kokras, N., Sfikakis, A., \& Papadopoulou-Daifoti, Z. (2005). Chronic mild stress impact: are females more vulnerable? Neuroscience, 135(3), 703-714. doi: 10.1016/j.neuroscience.2005.06.068

16. Dalla, C., Antoniou, K., Kokras, N., Drossopoulou, G., Papathanasiou, G., Bekris, S., . . PapadopoulouDaifoti, Z. (2008). Sex differences in the effects of two stress paradigms on dopaminergic neurotransmission. Physiol Behav, 93(3), 595-605. doi: 10.1016/j.physbeh.2007.10.020

17. Daniel, J. M., Hulst, J. L., \& Lee, C. D. (2005). Role of hippocampal M2 muscarinic receptors in the estrogen-induced enhancement of working memory. Neuroscience, 132(1), 57-64. doi: 10.1016/j.neuroscience.2005.01.002

18. Dimopoulos, N., Piperi, C., Salonicioti, A., Mitsonis, C., Liappas, I., Lea, R. W., \& Kalofoutis, A. (2006). Elevation of plasma concentration of adhesion molecules in late-life depression. Int J Geriatr Psychiatry, 21(10), 965-971. doi: 10.1002/gps.1592

19. Ferrer, M., Meyer, M., \& Osol, G. (1996). Estrogen replacement increases beta-adrenoceptor-mediated relaxation of rat mesenteric arteries. J Vasc Res, 33(2), 124-131. 
20. Gallucci, W. T., Baum, A., Laue, L., Rabin, D. S., Chrousos, G. P., Gold, P. W., \& Kling, M. A. (1993). Sex differences in sensitivity of the hypothalamic-pituitary-adrenal axis. Health Psychol, 12(5), 420-425.

21. Gilligan, D. M., Badar, D. M., Panza, J. A., Quyyumi, A. A., \& Cannon, R. O., 3rd. (1994). Acute vascular effects of estrogen in postmenopausal women. Circulation, 90(2), 786-791.

22. Goldstein, J. M., Jerram, M., Abbs, B., Whitfield-Gabrieli, S., \& Makris, N. (2010). Sex differences in stress response circuitry activation dependent on female hormonal cycle. J Neurosci, 30(2), 431-438. doi: 10.1523/JNEUROSCI.3021-09.2010

23. Grippo, A. J., Sullivan, N. R., Damjanoska, K. J., Crane, J. W., Carrasco, G. A., Shi, J., . . Van de Kar, L. D. (2005). Chronic mild stress induces behavioral and physiological changes, and may alter serotonin $1 \mathrm{~A}$ receptor function, in male and cycling female rats. Psychopharmacology (Berl), 179(4), 769-780. doi: 10.1007/s00213-004-2103-4

24. Haroon, E., Raison, C. L., \& Miller, A. H. (2012). Psychoneuroimmunology meets neuropsychopharmacology: translational implications of the impact of inflammation on behavior. Neuropsychopharmacology, 37(1), 137-162. doi: 10.1038/npp.2011.205

25. Isingrini, E., Belzung, C., Freslon, J. L., Machet, M. C., \& Camus, V. (2012). Fluoxetine effect on aortic nitric oxide-dependent vasorelaxation in the unpredictable chronic mild stress model of depression in mice. Psychosom Med, 74(1), 63-72. doi: 10.1097/PSY.0b013e31823a43e0

26. Isingrini, E., Surget, A., Belzung, C., Freslon, J. L., Frisbee, J., O'Donnell, J., . . d'Audiffret, A. (2011). Altered aortic vascular reactivity in the unpredictable chronic mild stress model of depression in mice: UCMS causes relaxation impairment to ACh. Physiol Behav, 103(5), 540-546. doi: 10.1016/j.physbeh.2011.04.002

27. Kamper, E. F., Chatzigeorgiou, A., Tsimpoukidi, O., Kamper, M., Dalla, C., Pitychoutis, P. M., \& Papadopoulou-Daifoti, Z. (2009). Sex differences in oxidant/antioxidant balance under a chronic mild stress regime. Physiol Behav, 98(1-2), 215-222. doi: 10.1016/j.physbeh.2009.05.011

28. Kendler, K. S., Gatz, M., Gardner, C. O., \& Pedersen, N. L. (2006). A Swedish national twin study of lifetime major depression. Am J Psychiatry, 163(1), 109-114. doi: 10.1176/appi.ajp.163.1.109 
29. Kessler, R. C., Berglund, P., Demler, O., Jin, R., Koretz, D., Merikangas, K. R., . . National Comorbidity Survey, R. (2003). The epidemiology of major depressive disorder: results from the National Comorbidity Survey Replication (NCS-R). JAMA, 289(23), 3095-3105. doi: 10.1001/jama.289.23.3095

30. Kessler, R. C., McGonagle, K. A., Swartz, M., Blazer, D. G., \& Nelson, C. B. (1993). Sex and depression in the National Comorbidity Survey. I: Lifetime prevalence, chronicity and recurrence. J Affect Disord, 29(2-3), 85-96.

31. Konkle, A. T., Baker, S. L., Kentner, A. C., Barbagallo, L. S., Merali, Z., \& Bielajew, C. (2003). Evaluation of the effects of chronic mild stressors on hedonic and physiological responses: sex and strain compared. Brain Res, 992(2), 227-238.

32. Krishnan, V., \& Nestler, E. J. (2010). Linking molecules to mood: new insight into the biology of depression. Am J Psychiatry, 167(11), 1305-1320. doi: 10.1176/appi.ajp.2009.10030434

33. Lambert, G., Johansson, M., Agren, H., \& Friberg, P. (2000). Reduced brain norepinephrine and dopamine release in treatment-refractory depressive illness: evidence in support of the catecholamine hypothesis of mood disorders. Arch Gen Psychiatry, 57(8), 787-793.

34. Lett, H. S., Blumenthal, J. A., Babyak, M. A., Sherwood, A., Strauman, T., Robins, C., \& Newman, M. F. (2004). Depression as a risk factor for coronary artery disease: evidence, mechanisms, and treatment. Psychosom Med, 66(3), 305-315.

35. Lloyd-Jones, D., Adams, R. J., Brown, T. M., Carnethon, M., Dai, S., De Simone, G., . . Stroke Statistics, S. (2010). Executive summary: heart disease and stroke statistics--2010 update: a report from the American Heart Association. Circulation, 121(7), 948-954. doi: 10.1161/CIRCULATIONAHA.109.192666

36. Lucca, G., Comim, C. M., Valvassori, S. S., Reus, G. Z., Vuolo, F., Petronilho, F., . . Quevedo, J. (2009). Effects of chronic mild stress on the oxidative parameters in the rat brain. Neurochem Int, 54(5-6), 358-362. doi: 10.1016/j.neuint.2009.01.001

37. MacRitchie, A. N., Jun, S. S., Chen, Z., German, Z., Yuhanna, I. S., Sherman, T. S., \& Shaul, P. W. (1997). Estrogen upregulates endothelial nitric oxide synthase gene expression in fetal pulmonary artery endothelium. Circ Res, 81(3), 355-362. 
38. Moreau, K. L., Donato, A. J., Tanaka, H., Jones, P. P., Gates, P. E., \& Seals, D. R. (2003). Basal leg blood flow in healthy women is related to age and hormone replacement therapy status. $J$ Physiol, 547(Pt 1), 309316. doi: 10.1113/jphysiol.2002.032524

39. Munhoz, C. D., Garcia-Bueno, B., Madrigal, J. L., Lepsch, L. B., Scavone, C., \& Leza, J. C. (2008). Stressinduced neuroinflammation: mechanisms and new pharmacological targets. Braz J Med Biol Res, 41(12), 1037-1046.

40. Nakagawa, K., Marji, J. S., Schwartzman, M. L., Waterman, M. R., \& Capdevila, J. H. (2003). Androgenmediated induction of the kidney arachidonate hydroxylases is associated with the development of hypertension. Am J Physiol Regul Integr Comp Physiol, 284(4), R1055-1062. doi: 10.1152/ajpregu.00459.2002

41. Patel, V., Garrison, P., de Jesus Mari, J., Minas, H., Prince, M., Saxena, S., \& Advisory group of the Movement for Global Mental, H. (2008). The Lancet's series on global mental health: 1 year on. Lancet, 372(9646), 1354-1357. doi: 10.1016/S0140-6736(08)61556-1

42. Peng, Y. L., Liu, Y. N., Liu, L., Wang, X., Jiang, C. L., \& Wang, Y. X. (2012). Inducible nitric oxide synthase is involved in the modulation of depressive behaviors induced by unpredictable chronic mild stress. J Neuroinflammation, 9, 75. doi: 10.1186/1742-2094-9-75

43. Pinto, V. L., de Souza, P. F., Brunini, T. M., Oliveira, M. B., Moss, M. B., Siqueira, M. A., . . MendesRibeiro, A. C. (2012). Low plasma levels of L-arginine, impaired intraplatelet nitric oxide and platelet hyperaggregability: implications for cardiovascular disease in depressive patients. J Affect Disord, 140(2), 187-192. doi: 10.1016/j.jad.2012.02.008

44. Pizzi, C., Manzoli, L., Mancini, S., Bedetti, G., Fontana, F., \& Costa, G. M. (2010). Autonomic nervous system, inflammation and preclinical carotid atherosclerosis in depressed subjects with coronary risk factors. Atherosclerosis, 212(1), 292-298. doi: 10.1016/j.atherosclerosis.2010.04.038

45. Plante, G. E. (2005). Depression and cardiovascular disease: a reciprocal relationship. Metabolism, 54(5 Suppl 1), 45-48. doi: 10.1016/j.metabol.2005.01.013

46. Porsolt, R. D. (2000). Animal models of depression: utility for transgenic research. Rev Neurosci, 11(1), 5358. 
47. Raison, C. L., \& Miller, A. H. (2003). When not enough is too much: the role of insufficient glucocorticoid signaling in the pathophysiology of stress-related disorders. Am J Psychiatry, 160(9), 1554-1565.

48. Rajagopalan, S., Brook, R., Rubenfire, M., Pitt, E., Young, E., \& Pitt, B. (2001). Abnormal brachial artery flow-mediated vasodilation in young adults with major depression. Am J Cardiol, 88(2), 196-198, A197.

49. Rivier, C., \& Vale, W. (1985). Effect of the long-term administration of corticotropin-releasing factor on the pituitary-adrenal and pituitary-gonadal axis in the male rat. J Clin Invest, 75(2), 689-694. doi: 10.1172/JCI111748

50. Rupnow, H. L., Phernetton, T. M., Shaw, C. E., Modrick, M. L., Bird, I. M., \& Magness, R. R. (2001). Endothelial vasodilator production by uterine and systemic arteries. VII. Estrogen and progesterone effects on eNOS. Am J Physiol Heart Circ Physiol, 280(4), H1699-1705.

51. Scott, P. A., Tremblay, A., Brochu, M., \& St-Louis, J. (2007). Vasorelaxant action of 17 -estradiol in rat uterine arteries: role of nitric oxide synthases and estrogen receptors. Am J Physiol Heart Circ Physiol, 293(6), H3713-3719. doi: 10.1152/ajpheart.00736.2007

52. Shaw, L. J., Bairey Merz, C. N., Azziz, R., Stanczyk, F. Z., Sopko, G., Braunstein, G. D., . . Pepine, C. J. (2008). Postmenopausal women with a history of irregular menses and elevated androgen measurements at high risk for worsening cardiovascular event-free survival: results from the National Institutes of Health-National Heart, Lung, and Blood Institute sponsored Women's Ischemia Syndrome Evaluation. J Clin Endocrinol Metab, 93(4), 1276-1284. doi: 10.1210/jc.2007-0425

53. Singh, H., \& Schwartzman, M. L. (2008). Renal vascular cytochrome P450-derived eicosanoids in androgeninduced hypertension. Pharmacol Rep, 60(1), 29-37.

54. Takahashi, M., Suzuki, E., Takeda, R., Oba, S., Nishimatsu, H., Kimura, K., . . . Hirata, Y. (2008). Angiotensin II and tumor necrosis factor-alpha synergistically promote monocyte chemoattractant protein-1 expression: roles of NF-kappaB, p38, and reactive oxygen species. Am J Physiol Heart Circ Physiol, 294(6), H2879-2888. doi: 10.1152/ajpheart.91406.2007 
55. Talboom, J. S., Williams, B. J., Baxley, E. R., West, S. G., \& Bimonte-Nelson, H. A. (2008). Higher levels of estradiol replacement correlate with better spatial memory in surgically menopausal young and middle-aged rats. Neurobiol Learn Mem, 90(1), 155-163. doi: 10.1016/j.nlm.2008.04.002

56. Tobet, S. A., Handa, R. J., \& Goldstein, J. M. (2013). Sex-dependent pathophysiology as predictors of comorbidity of major depressive disorder and cardiovascular disease. Pflugers Arch, 465(5), 585-594. doi: 10.1007/s00424-013-1248-6

57. Vamvakopoulos, N. V. (1995). Sexual dimorphism of stress response and immune/ inflammatory reaction: the corticotropin releasing hormone perspective. Mediators Inflamm, 4(3), 163-174. doi: $10.1155 / \mathrm{S} 0962935195000275$

58. Whiteford, H. A., Degenhardt, L., Rehm, J., Baxter, A. J., Ferrari, A. J., Erskine, H. E., . . Vos, T. (2013). Global burden of disease attributable to mental and substance use disorders: findings from the Global Burden of Disease Study 2010. Lancet, 382(9904), 1575-1586. doi: 10.1016/S0140-6736(13)61611-6

59. Willner, P. (1997). Validity, reliability and utility of the chronic mild stress model of depression: a 10-year review and evaluation. Psychopharmacology (Berl), 134(4), 319-329.

60. Yanes, L. L., \& Reckelhoff, J. F. (2011). Postmenopausal hypertension. Am J Hypertens, 24(7), 740-749. doi: 10.1038/ajh.2011.71

61. Young, E. A., Midgley, A. R., Carlson, N. E., \& Brown, M. B. (2000). Alteration in the hypothalamicpituitary-ovarian axis in depressed women. Arch Gen Psychiatry, 57(12), 1157-1162. 
Table 1. Baseline characteristics between mouse groups in the present study. All mice are aged 17-18 weeks. $* \mathrm{p}<0.05$ versus control.

\begin{tabular}{ccccc}
\hline & $\begin{array}{c}\text { Males } \\
(\mathbf{n = 1 4})\end{array}$ & $\begin{array}{c}\text { Females } \\
(\mathbf{n = 9})\end{array}$ & $\begin{array}{c}\text { UCMS-Males } \\
(\mathbf{n = 1 2})\end{array}$ & $\begin{array}{c}\text { UCMS-Females } \\
(\mathbf{n}=\mathbf{1 2})\end{array}$ \\
\hline Mass $(\mathrm{g})$ & $29 \pm 2$ & $28 \pm 3$ & $30 \pm 3$ & $29 \pm 4$ \\
MAP $(\mathrm{mmHg})$ & $87 \pm 4$ & $91 \pm 4$ & $94 \pm 5$ & $90 \pm 5$ \\
Insulin $_{\text {plasma }}(\mathrm{ng} / \mathrm{ml})$ & $1.1 \pm 0.3$ & $1.2 \pm 0.4$ & $4.1 \pm 0.7^{*}$ & $4.5 \pm 0.5^{*}$ \\
Glucose $_{\text {blood }}(\mathrm{mg} / \mathrm{dl})$ & $82 \pm 7$ & $80 \pm 8$ & $94 \pm 8$ & $101 \pm 10$ \\
Cholesterol $_{\text {plasma }}(\mathrm{mg} / \mathrm{dl})$ & $71 \pm 7$ & $68 \pm 8$ & $78 \pm 6$ & $77 \pm 10$ \\
Triglycerides $_{\text {plasma }}(\mathrm{mg} / \mathrm{dl})$ & $94 \pm 6$ & $101 \pm 8$ & $109 \pm 8$ & $116 \pm 12$ \\
Nitrotyrosine $_{\text {plasma }}(\mathrm{ng} / \mathrm{ml})$ & $12 \pm 3$ & $11 \pm 4$ & $29 \pm 5^{*}$ & $36 \pm 6^{*}$ \\
Cortisol $_{\text {plasma }}(\mathrm{pg} / \mathrm{ml})$ & $12 \pm 3$ & $14 \pm 4$ & $29 \pm 5^{*}$ & $44 \pm 4^{*}$ \\
TNF- $_{\text {plasma }}(\mathrm{pg} / \mathrm{ml})$ & $2.3 \pm 0.3$ & $2.0 \pm 0.2$ & $4.1 \pm 0.4^{*}$ & $6.4 \pm 0.4^{*} \dagger$ \\
MCP- $_{\text {plasma }}(\mathrm{pg} / \mathrm{ml})$ & $2.8 \pm 0.3$ & $3.3 \pm 0.5$ & $10.2 \pm 1.0^{*}$ & $14.8 \pm 1.3^{* \dagger}$ \\
\hline
\end{tabular}




\section{FIGURE LEGENDS}

Figure 1. Depressive symptoms following eight weeks of the UCMS protocol in male and female BALB/cJ mice. Data are presented for coat status (Panel A), the latency (Panel B) and frequency (Panel C) of facial grooming following a $10 \%$ sucrose solution spray, and the total period of immobility during the tail suspension test (Panel D) for control and UCMS mice. Control males $n=14$; Control females $n=9$; UCMS males $n=12$, UCMS females $n=12$. * $\mathrm{p}<0.05$ versus Control in the sex; $\uparrow \mathrm{p}<0.05$ versus UCMS-Male.

Figure 2. Constrictor responses of aortic rings to increasing concentrations of phenylephrine from mice under control conditions and after eight weeks of UCMS. Data are presented under untreated conditions (Panel A) and in response to NOS inhibition with L-NAME (Panel B). Control males n=12; Control females n=8; UCMS males n=10, UCMS females $n=10$.

Figure 3. Dilator responses of aortic rings to increasing concentrations of methacholine from mice under control conditions and after eight weeks of UCMS. Panel A presents data under untreated conditions. Panels B and C present data describing dilator responses from male and female Control mice, respectively, following pre-treatment with L-NAME, indomethacin or combination. Panels D and E present data describing dilator responses from male and female UCMS mice, respectively, following pre-treatment with L-NAME, indomethacin or combination. Control males $n=6-9$; Control females $n=5-7$; UCMS males $n=8-10$, UCMS females $n=7-10 . * p<0.05$ versus responses in untreated vascular rings from control mice of that sex. $\uparrow \mathrm{p}<0.05$ versus responses in untreated vascular rings from UCMS-male mice.

Figure 4. Dilator responses of aortic rings to increasing concentrations of sodium nitroprusside from mice under control conditions and after eight weeks of UCMS. Control males $n=10$; Control females $n=8$; UCMS males $n=9$, UCMS females $n=9$. 
Figure 5. Vascular production of nitric oxide (Panel A), PGI $_{2}$ (Panel B; estimated from 6-keto-PGF ${ }_{1 \alpha}$ ), and $\mathrm{TxA}_{2}$ (Panel $\mathrm{C}$; estimated from 11-dehydro- $\left.\mathrm{TxB}_{2}\right)$ following methacholine challenge in mice under control conditions and following eight weeks of UCMS. Control males $n=9$; Control females $n=7$; UCMS males $n=8$, UCMS females $\mathrm{n}=8$. * $\mathrm{p}<0.05$ versus responses in vessels from Control mice, $\uparrow \mathrm{p}<0.05$ versus responses in vessels from UCMS-Males.

Figure 6. Vascular production of nitric oxide following methacholine challenge in male and female mice following eight weeks of UCMS. Data are presented for vessels under control (untreated) conditions, following treatment of vessels from UCMS animals with TEMPOL and following treatment of vessels from UCMS animals with L-NAME. Control males $n=9$; Control females $n=7$; UCMS males $n=8$, UCMS females $n=8$. * $p<0.05$ versus responses in Control vessels from mice of that sex, $\uparrow \mathrm{p}<0.05$ versus responses in untreated vessels of UCMS mice of that sex, $\$ p<0.05$ versus responses in UCMS+TEMPOL treated mice of that sex.

Figure 7. Dilator responses of aortic rings to increasing concentrations of methacholine from UCMS-Male (Panel A) and UCMS-Female (Panel B) mice. Panel A presents data from UCMS-Males under untreated conditions, following pre-treatment with TEMPOL, following treatment with TEMPOL/INDO, and following treatment with TEMPOL/LNAME. Panel B presents data from UCMS-Females under untreated conditions, following pre-treatment with TEMPOL, following treatment with TEMPOL/INDO and following treatment with TEMPOL/L-NAME. Control males $\mathrm{n}=8-11$; Control females $\mathrm{n}=7-9$; UCMS males $\mathrm{n}=8-9$, UCMS females $\mathrm{n}=7-9$. $* \mathrm{p}<0.05$ versus responses in untreated vessels, $\uparrow \mathrm{p}<0.05$ versus responses in vessels that were pre-treated with TEMPOL.

Figure 8. Constrictor responses of gracilis arterioles to increasing concentrations of phenylephrine from mice under control conditions and after eight weeks of UCMS. Data are presented under control conditions (Panel A) and in response to NOS inhibition with L-NAME (Panel B). Control males n=10; Control females n=8; UCMS males n=10, UCMS females $\mathrm{n}=10 . * \mathrm{p}<0.05$ versus responses in arterioles from Control mice. 
Figure 9. Dilator responses of gracilis arterioles to increasing concentrations of acetylcholine from mice under control conditions and after eight weeks of UCMS. Panel A presents data from male mice under untreated conditions and following pre-treatment with L-NAME or indomethacin. Panel B presents data from male mice after pretreatment with TEMPOL alone or with L-NAME. Panel C presents data from female mice under untreated conditions and following pre-treatment with L-NAME or indomethacin. Panel D presents data from female mice after pretreatment with TEMPOL alone or with L-NAME. Control males n=8-10; Control females $n=7-8$; UCMS males n=910, UCMS females $\mathrm{n}=8-10$. * $\mathrm{p}<0.05$ versus responses in vessels from Control mice of that sex; $\uparrow \mathrm{p}<0.05$ versus responses in vessels from UCMS mice of that sex.

Figure 10. Correlations between vascular reactivity and individual markers of chronic inflammation in control male (blue), control female (red), UCMS male (green) and UCMS female (black) mice. Data are presented as the lower bound of the methacholine concentration-response curve for an individual aortic ring versus plasma concentrations of TNF- $\alpha$ (Panel A) or MCP-1 (Panel B). In this figure, the lower bound from methacholine concentration-response curve is reflective of the degree of dilator reactivity for that vessel. If the vessel is more reactive to methacholine, the lower bound is decreased, while if the vessel becomes is less reactive, the lower bound is increased. Also presented are lines of best fit through the data for each group, with the resulting slope $(\beta)$ and $r^{2}$ values presented in the legend. 

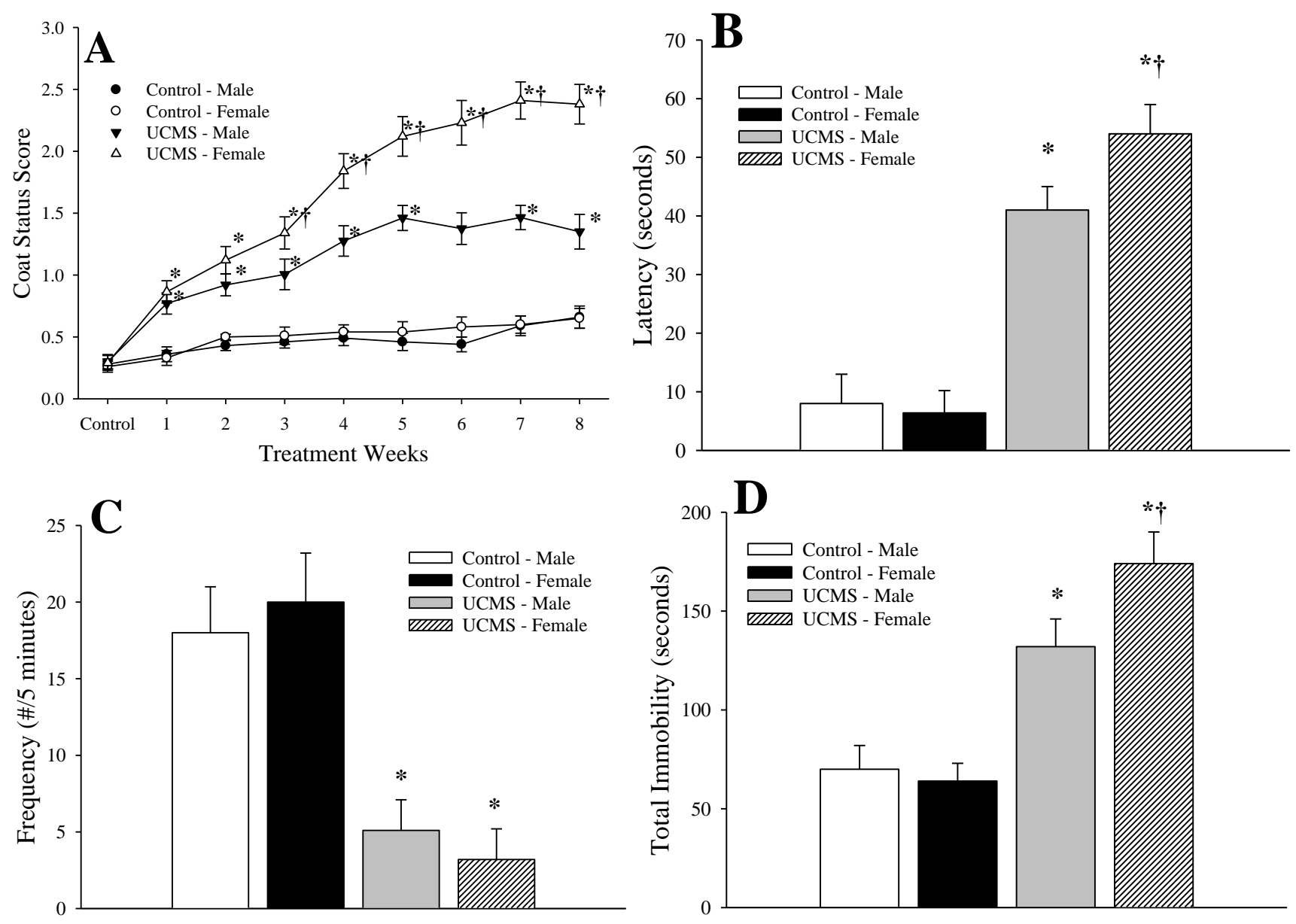

Figure 1.

Stanley et al. 

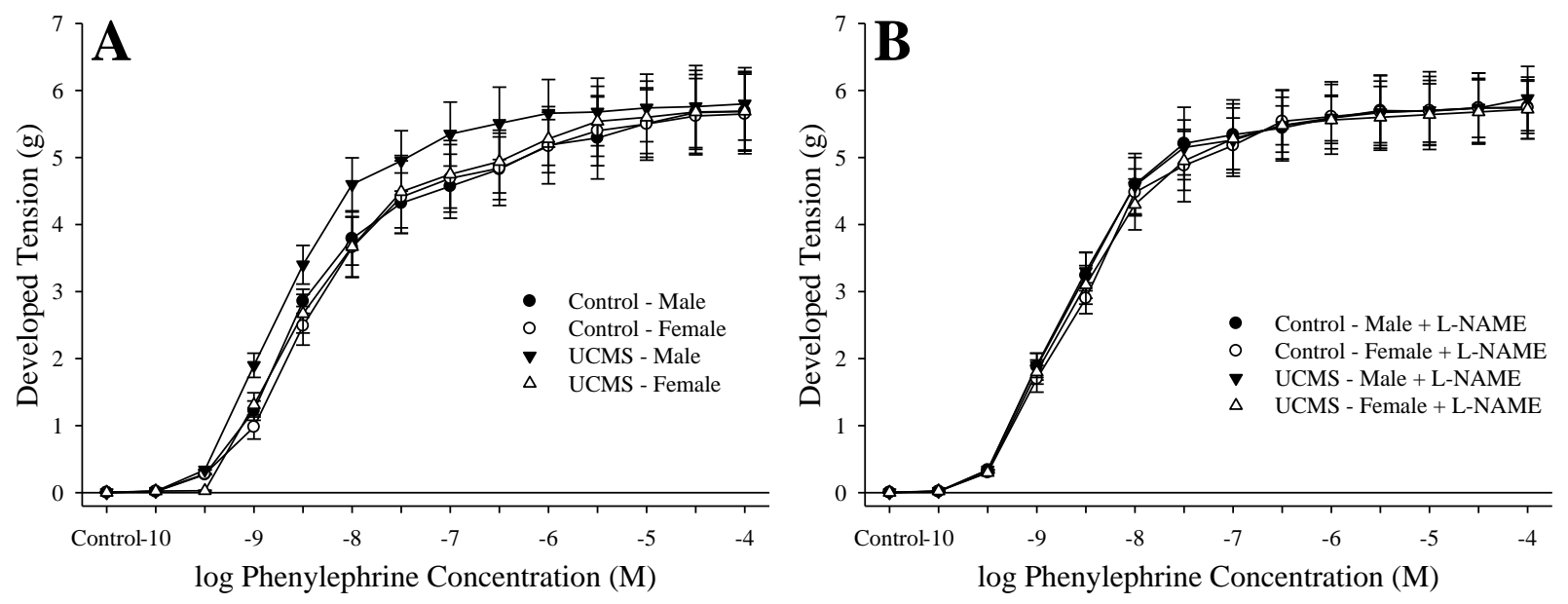

Figure 2.

Stanley et al. 

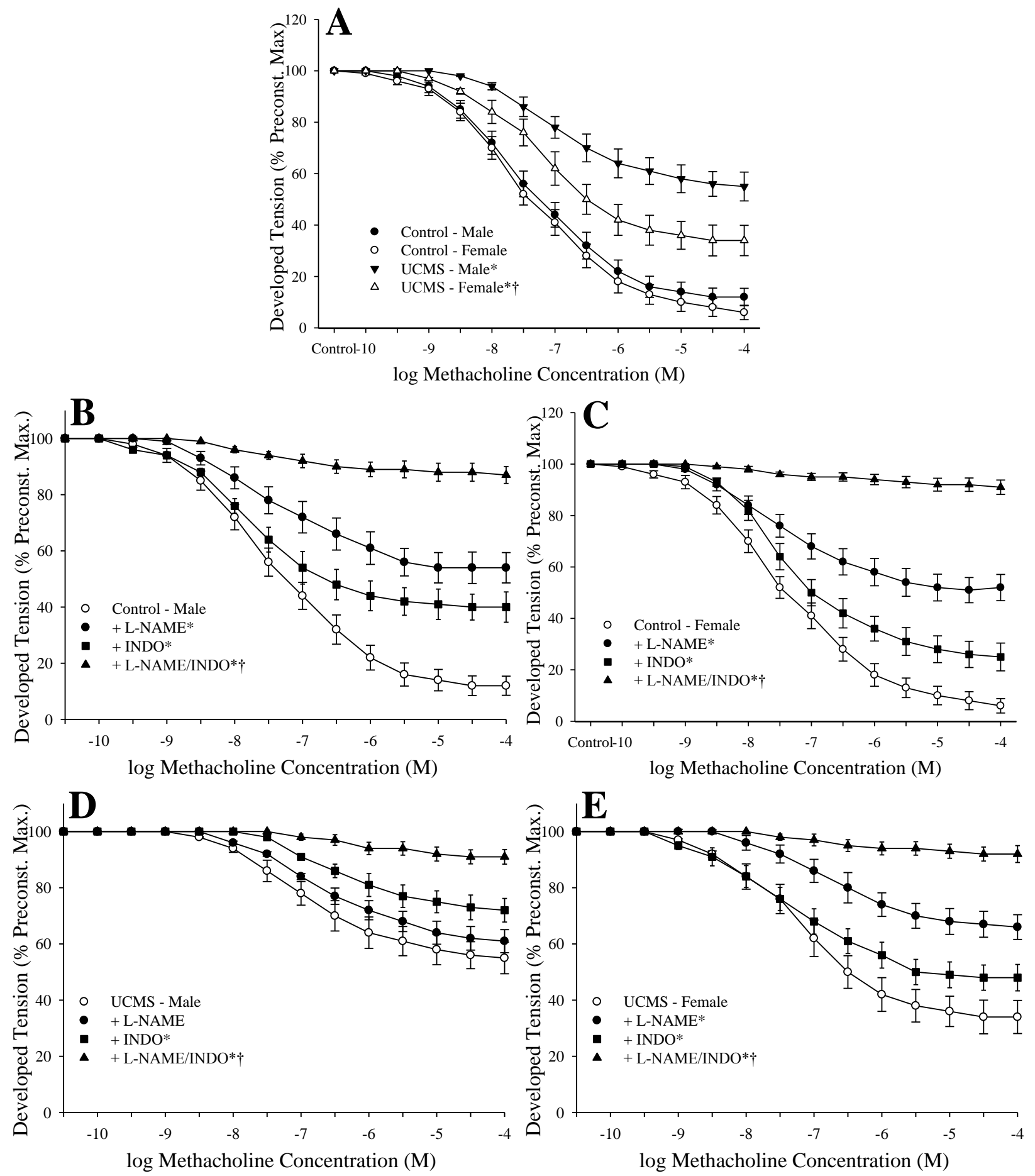

Figure 3.

Stanley et al. 


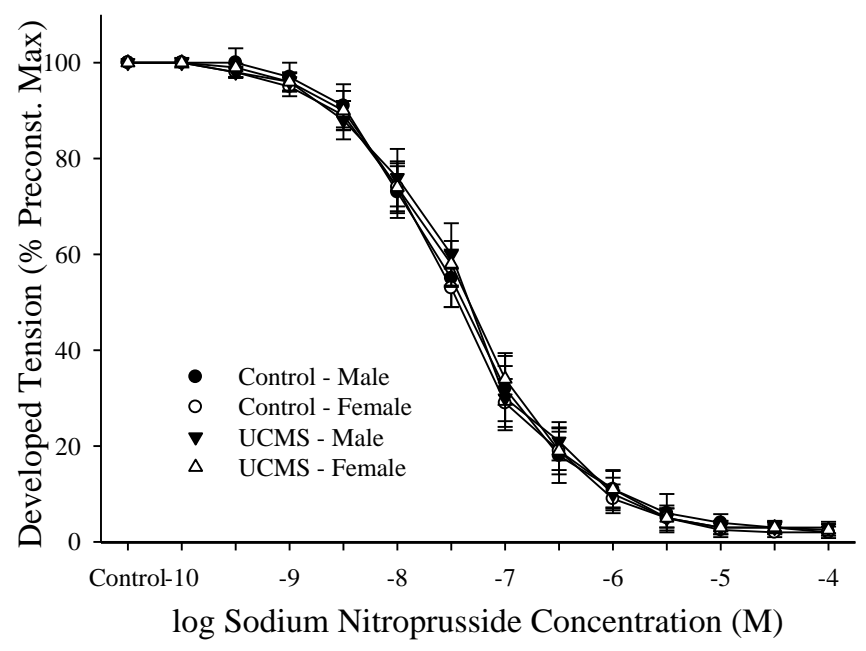

Figure 4

Stanley et al.

55 

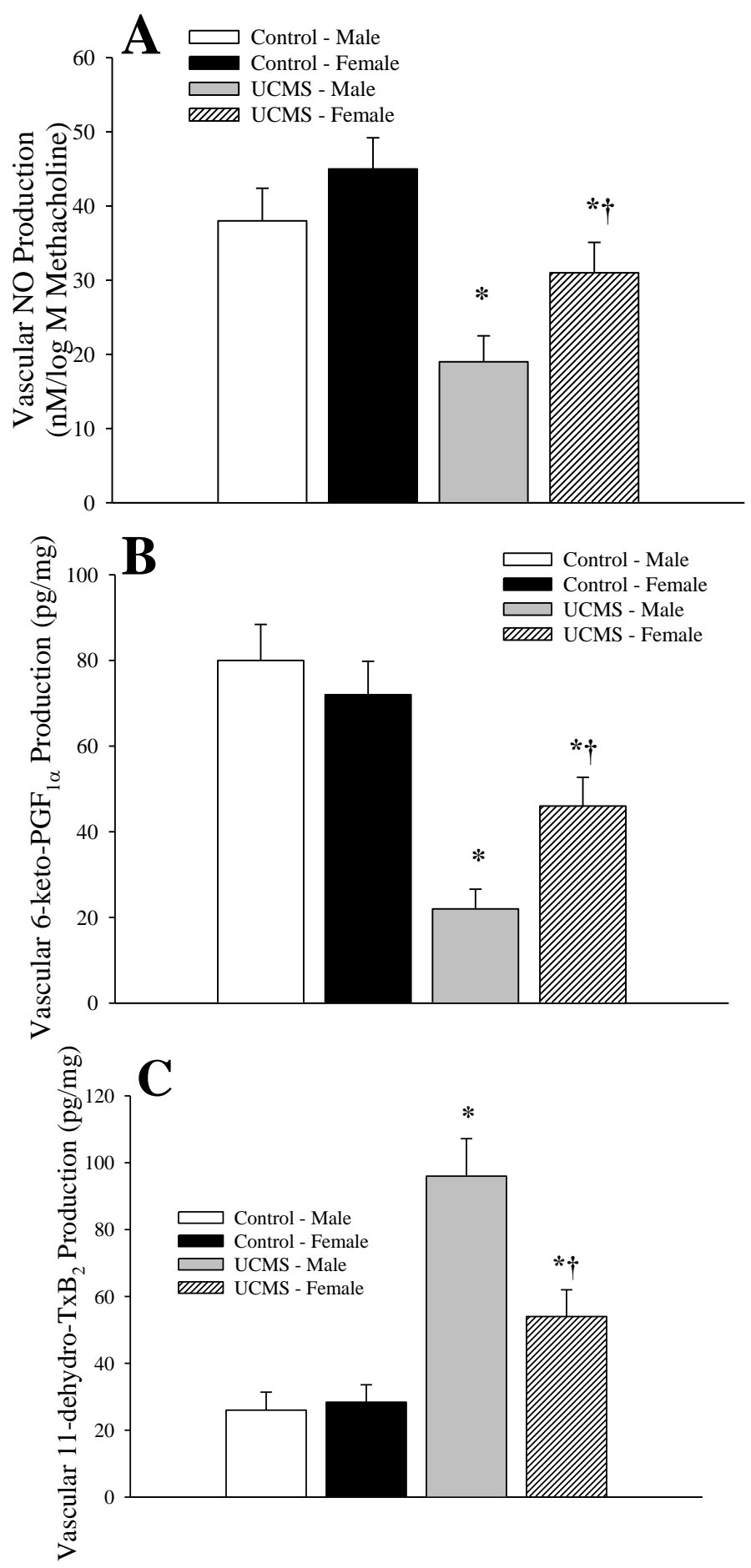

Figure 5

Stanley et al. 


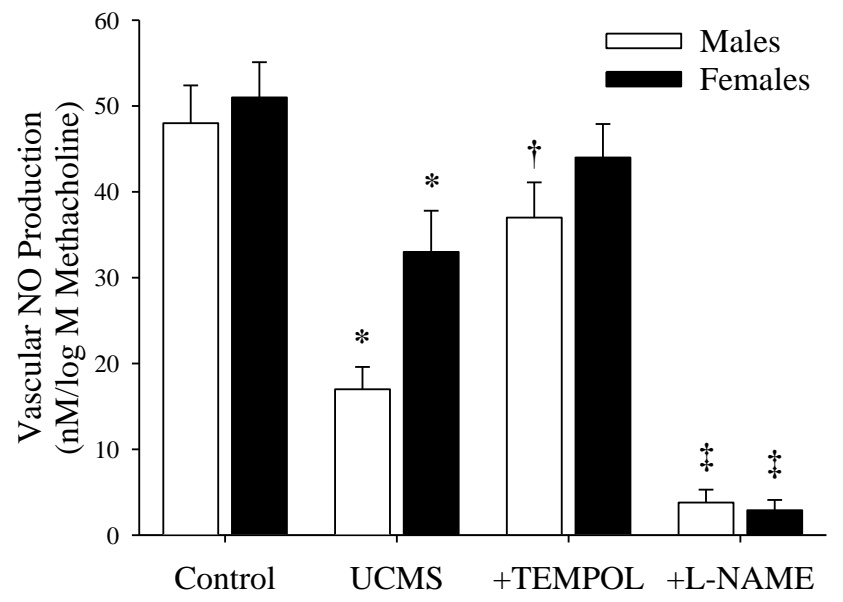

Figure 6

Stanley et al. 

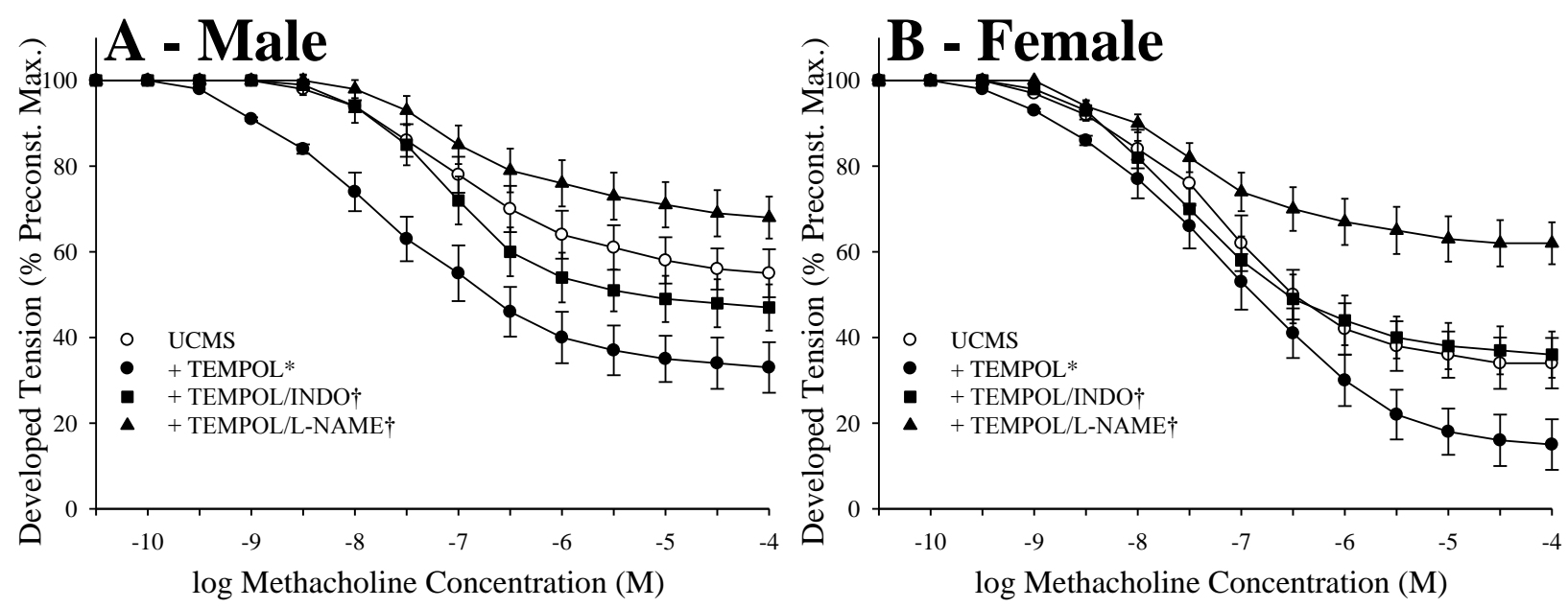

Figure 7

Stanley et al. 

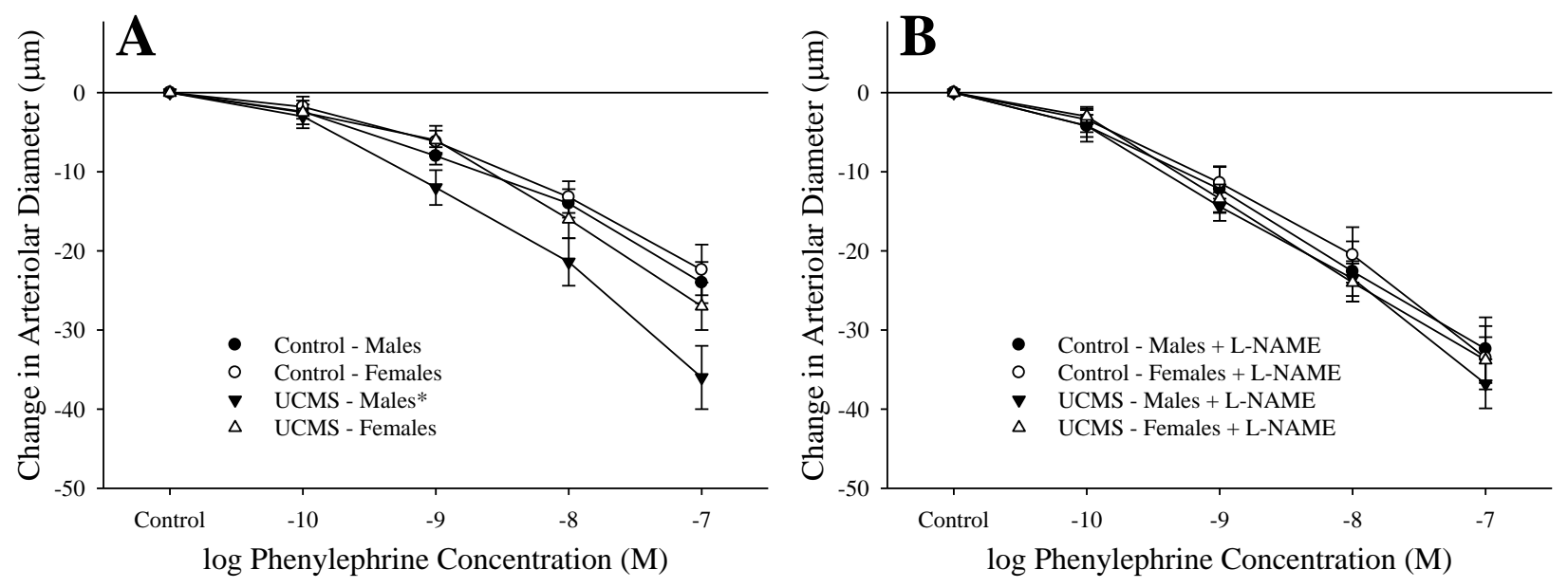

Figure 8

Stanley et al. 

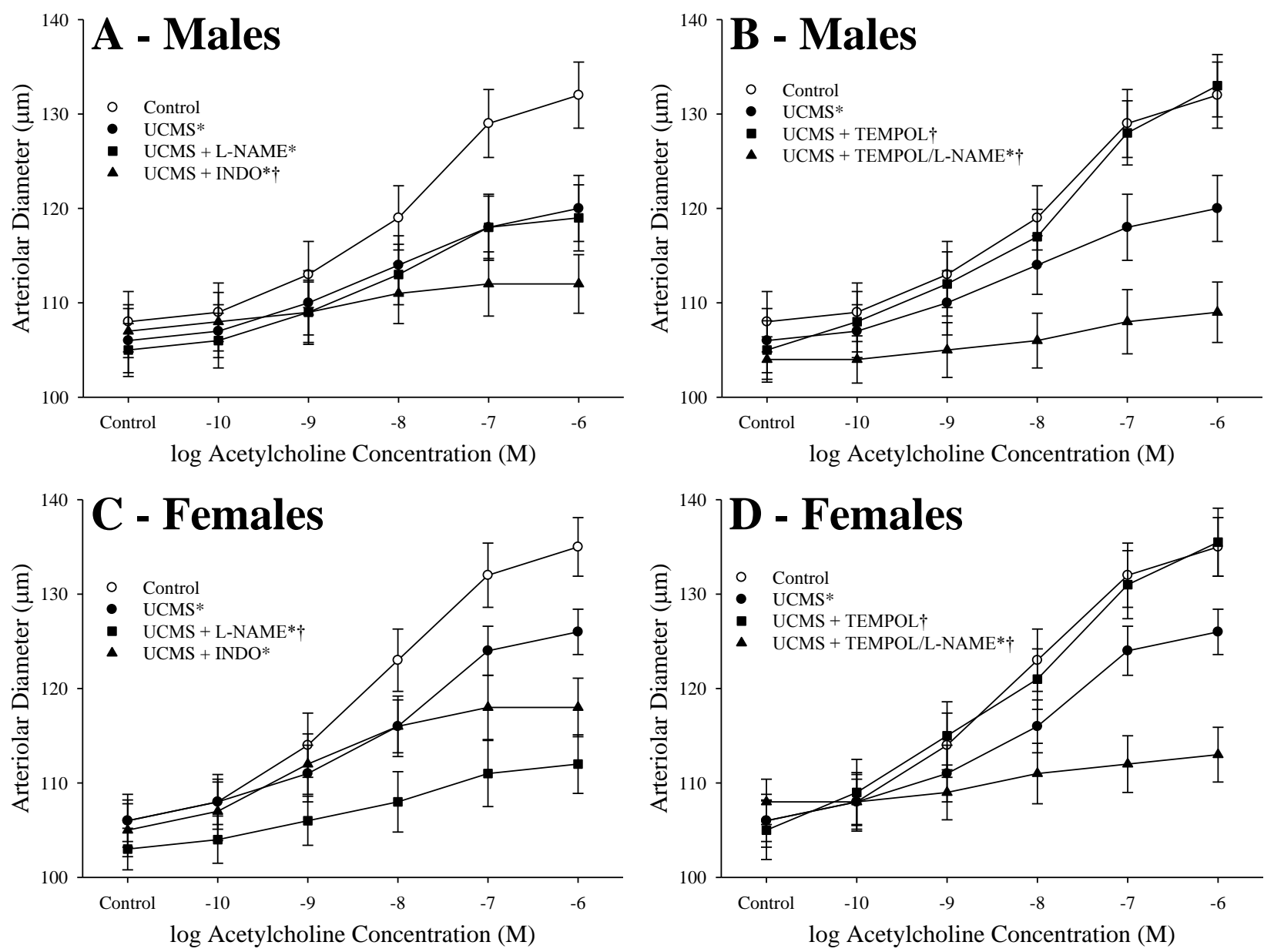

\section{Figure 9}

Stanley et al. 


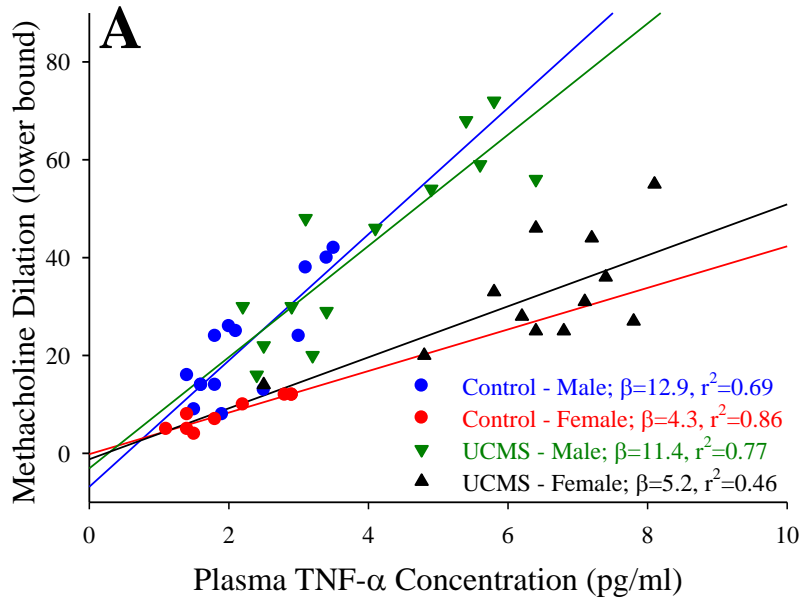

Plasma TNF- $\alpha$ Concentration (pg/ml)

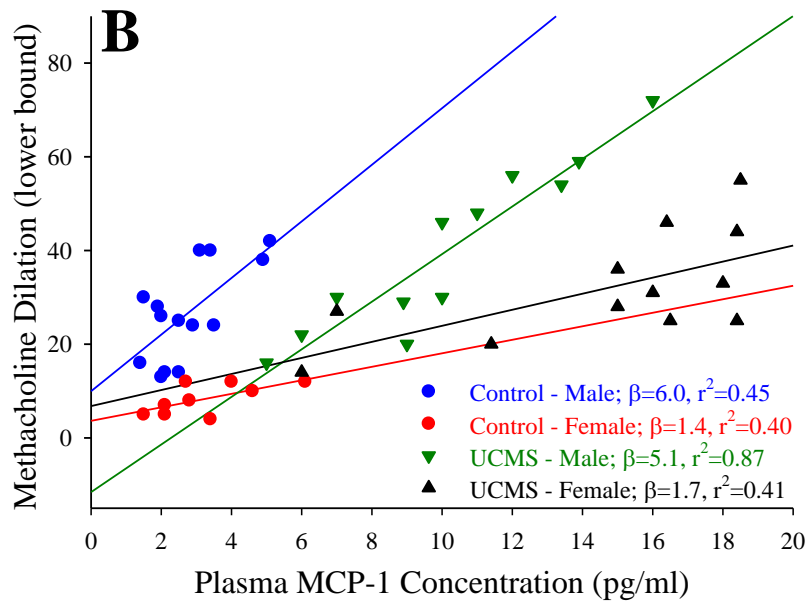

Figure 10

Stanley et al. 


\section{CHAPTER 1: CONCEPTUAL FRAMEWORK}

The conclusions from the study presented in this chapter demonstrated several novel findings and also introduced three main questions, which are addressed in chapter 2 of this dissertation. The main findings can be briefly summarized as follows:

- Unpredictable chronic mild stress- induced graded outcomes were associated with the severity of depressive symptom development that differed between male and female BALBc/J mice. Specifically, female animals exhibited a greater susceptibility to behavioral symptoms as measured by worsened coat score, increased immobility in tail suspension, decreased sucrose-stimulated grooming, and elevated cortisol levels. In addition, UCMS induced endothelial dysfunction in aortic rings and gracilis arterioles as evidenced by decreased vasodilator response to agonist challenge, as well as reduced $\mathrm{NO}$ and $\mathrm{PGI}_{2}$ bioavailability, and increased $\mathrm{Tx} \mathrm{A}_{2}$ production. However, these impairments to vascular reactivity were more modest in UCMS females despite worsened behavioral outcomes as well as greater levels of oxidative stress and inflammatory biomarkers compared to UCMS males.

- Comparable levels of either TNF- $\alpha$ and MCP-1 was associated with a greater degree of vascular dysfunction in males compared to females, and this relationship was observed in control as well as UCMS animals. In addition, there was a shift of balance in $\mathrm{COX}$ metabolism, with a greater decrease in $\mathrm{PGI}_{2}$ and subsequent increase in $\mathrm{TxA}_{2}$ production observed in UCMS males.

- Using female mice that were randomized with respect to their time in estrous cycle allowed investigation of potential interactions between the sex steroid hormones during the normal ovulatory cycle, when a milieu of sex steroid hormones are present and fluctuating in a coordinated manner. This approach showed a longer, more temporally stable protective effect of female characteristics rather than one that reflects the labile changes in hormone status.

These observations led to the following questions, which form the basis of the next chapter of this dissertation. Chapter 2 will focus on a enhancing the translational relevance of the study as further investigations of potential sex-specific mechanisms linking chronic stress, depressive symptoms, and peripheral vascular dysfunction continue. 
1. To increase the translational value of the experimental model, rats will be used for the remainder of the projects. The mechanisms and pathology of disease in rats is more comparable to that of humans. Will the sex specific functional outcomes utilizing the same relevant model of stress-induced depression reflect similar findings using a new species model?

2. Given the sex disparities to UCMS seen in mice regarding protection of vascular function, what roles do female sex hormones play in conferring protection against endothelial dysfunction?

3. Given the prevalence and increased risk of depression in individuals with CVD, what impact would UCMS have on behavioral outcomes and vascular function in an animal model with pre-existing CVD risk factors?

The next chapter of this dissertation sought to answer these three questions in the Obese Zucker rat (OZR), a model of metabolic syndrome. The OZR, with its inherent development of obesity, insulin resistance, moderate hypertension, and hyperlipidemia represents an excellent model of CVD risk, and while there have been numerous studies describing alterations to vascular reactivity and microvascular network structure/mechanics in OZR, there is limited data addressing the impact of chronic stress/depressive symptoms in an integrated system of pre-existing poor CVD health. Thus, both male and female OZR, along with their lean controls, were used to investigate the effects of comorbid metabolic syndrome and UCMS on behavioral and vascular outcomes. A separate cohort of ovariectomized female LZR and OZR was included to specifically investigate the impact of estrogen deficiency on physiologic stress response in female animals. 


\section{Chapter 2:}

THE ROLE OF FEMALE SEX HORMONES IN MODULATING STRESS-INDUCED VASCULAR DYSFUNCTION IN LEAN AND OBESE ZUCKER RATS

Shyla C. Stanley ${ }^{1,4}$, Steven D. Brooks ${ }^{1,3,4}$, Camille M Leon ${ }^{1,4}$, Paulina R Skaff ${ }^{1,4}$, Whitney Poling ${ }^{1,4}$, Alexandre C. d'Audiffret ${ }^{2,3,4}$, and Jefferson C. Frisbee ${ }^{1,3,4}$

\footnotetext{
Department of Physiology and Pharmacology ${ }^{1}$, Division of Vascular and Endovascular Surgery ${ }^{2}$, Clinical and Translational Sciences Institute ${ }^{3}$, Center for Cardiovascular and Respiratory Sciences ${ }^{4}$; West Virginia University Health Sciences Center, Morgantown, WV
}

Running Head: Sex Disparities, Depressive Symptoms and Vascular Function

Send Correspondence to:

Jefferson C. Frisbee, Ph.D.,

Center for Cardiovascular and Respiratory Sciences

Department of Physiology and Pharmacology

Robert C. Byrd Health Sciences Center

PO Box 9105

West Virginia University School of Medicine

Morgantown, WV 26505

Phone: (304) 293-6527

Fax: (304) 293-5513

Email: jefrisbee@hsc.wvu.edu 


\section{ABSTRACT}

The prevalence and severity of depression is tightly linked to development of cardiovascular disease (CVD) and cardiovascular events; the steadily rising incidence of comorbid pathology exacerbates negative cardiovascular outcome and greatly impacts the efficacy of clinical treatments/interventions. Furthermore, the marked sex disparity in incidence, clinical presentation, and health outcomes of depression and CVD presents an additional significant hurdle for public health practices. The unpredictable chronic mild stress (UCMS) protocol is a translationally relevant model for imposing depressive symptoms in rodents, resulting in sex specific behavioral and physiological outcomes. Sex specific effects have been attributed to female hormones, specifically, a cardioprotective effect of estrogen that mitigate UCMS induced vasculopathy. Therefore, the effects of UCMS in females lacking ovarian hormone production (OVX) were assessed in both healthy rats and in genetically modified rats that are pre-disposed to metabolic syndrome. The results suggest that sex hormones are differentially regulated in female rats as a result of pre-existing CVD risk factors that alter lipid profiles and inflammatory conditions that exacerbate negative health outcomes. Additionally, comorbid depression and CVD altered the behavioral outcomes in male and female animals compared to healthy controls.

Key Words: vasodilation, endothelial dysfunction, chronic stress, clinical depression, sex disparities, metabolic syndrome, cardiovascular risk factors 


\section{INTRODUCTION}

Cardiovascular disease is the leading cause of death for both men and women in the United States (Center for Disease Control). Over the past decade, an extensive body of clinical evidence has identified depression as a predictor of CVD risk factors that are independent of other traditional cardiovascular risk factors in patients with or without overt CVD (Patel et al., 2008; Plante et al., 2005; Whiteford et al., 2013). Substantial evidence indicates that chronic stress, a major contributing factor in depression onset, is a significant driving factor in the development of CVD and associated antecedent risk factors (Bayramgurler et al., 2013; Barden et al., 2004; Beck et al., 2002) while also demonstrating more predictive of CVD than traditional CV risk factors. (Everson-Rose et al., 2005). Chronic stress- induced dysfunction of the physiologic stress response systems, the sympatheticadrenal-medullary (SAM) and hypothalamic-pituitary-adrenal (HPA) axes, causes sex-specific pathophysiological events strongly linked to both depression and the development of CVD, including autonomic nervous system and immune dysfunction, as well as impairments to vascular reactivity and endothelial function.

The CDC and National Association of Mental Illness report that adult women are twice as likely to suffer from depression compared to men (Kuehner et al., 2003), with risk fluctuating in correspondence with hormone status (Steiner et al., 2003). However, premenopausal women have lower rates of major CV events compared to same-aged men, despite the presence of comparable CVD risk factors. Beginning at menopause, corresponding with reduced ovarian hormone production, CVD incidence rapidly rises; correspondingly, the presence of underlying risk factors including diabetes mellitus, hypertension, smoking, hypercholesterolemia, and obesity heighten risk of major CV events compared to same-aged men (Vaccarino et al., 1999; Bello and Mosca, 2004; Kenachaiah et al., 2004; Mähönen et al., 2004; Polk and Naqvi, 2005). The loss of sex-specific protective effects at menopause has largely been attributed to a substantial decline in ovarian production of hormones, specifically estrogen, which has major roles in conferring CV protection as well as antioxidant effects. Studies in chronically stressed female rodents have demonstrated a protective effect against stress-induced vasculopathy through preservation of endothelial function in both large and small arteries versus male counterparts (Stanley et al., 2014). However, the specific mechanisms governing the complex relationship between sex-specific vascular pathologies, chronic stress/depression, and general CVD risk factors are not well established. 
The unpredictable chronic mild stress (UCMS) procedure has been validated in behavioral studies as a highly relevant rodent model of clinical depression based upon its ability to reproduce symptoms of depression, including anhedonia and learned helplessness (Willner, 1997; Grippo, 2005). Importantly, the UCMS protocol is also ideally suited to study the vascular effects of chronic stress and depression. Animals subjected to UCMS exhibit impaired endothelial-dependent vasodilation associated with decreased nitric oxide (NO) bioavailability and increased pro-inflammatory, pro-oxidative conditions (d'Audiffret et al., 2010), similar to clinical signs of CVD (Isingrini et al., 2012; Willner et al., 1997). Studies utilizing the UCMS protocol have shown that female rodents have a greater susceptibility to depressive-like behaviors (Krishnan et al., 2010; Plante et al., 2005), but a less severe impairment of vascular endothelial function compared to male animals (Stanley et al., 2014). These positive, protective effects on vascular function in female animals during chronic stress conditions have been linked to specific estrogen actions (Dalla et al., 2005; Grippo et al., 2005; Wang et al., 2014) in promoting antioxidant defense and NO bioavailability that are necessary for normal endothelial function and vascular antiinflammatory and antioxidant capacities (Rupnow et al., 2001).

This purpose of this study is to further elucidate the role of female sex hormone (estrogen) in modulating the physiological response to chronic mild stressors, the development of depressive symptoms, and the corresponding development of stress-induced vascular dysfunction in female rodents with deficient estrogen levels as a result of ovariectomy (OVX). Furthermore, most studies to date have focused on the effects of chronic stress/depression in healthy animals, while investigation of these effects in models of comorbid cardiovascular risk factors are largely lacking. As such, this study will interrogate this complex bidirectional relationship between depression and CVD using an animal model exhibiting a complex state of poor cardiovascular health similar to that of human disease. To this end, the obese Zucker rat $\left(\mathrm{OZR}\right.$; Lepr $\left.{ }^{f a f a}\right)$ is a valid representative model of the cardiovascular risk factors that comprise the metabolic syndrome. Due to a mutation of the leptin receptor, the OZR develops chronic hyperphagia that progressively leads to development of moderate hypertension, hypercholesterolemia, hypertriglyceridemia, hyperglycemia, and insulin resistance, which are characterized in metabolic syndrome in human subjects. 
Eight weeks of UCMS were imposed on male, female, and ovariectomized female lean and obese Zucker rats (LZR, OZR) at 9 weeks of age to determine first, the effects of co-morbid chronic stress and CV risk on the development of depressive symptoms and vascular dysfunction in a model of the metabolic syndrome; and secondly, to investigate the impact of estrogen deficiency on vascular outcomes of chronic stress in both healthy females and females with comorbid metabolic syndrome.

\section{MATERIALS AND METHODS}

Animals: Male and female LZR and OZR (Harlan) were fed standard chow and drinking water ad libitum and were housed in the animal care facility at the WVU HSC. A separate cohort of ovariectomized (OVX) female LZR and OZR (Harlan) were similarly housed; OVX was performed by Harlan Laboratories prior to animal shipment. All protocols received prior IACUC approval. At nine weeks of age, rats from each sex were divided into two groups, control and UCMS (below). After eight weeks duration under either control or UCMS conditions, rats were anesthetized with injections of sodium pentobarbital $\left(50 \mathrm{mg} \bullet \mathrm{kg}^{-1}\right.$ i.p. $)$ and a carotid artery was cannulated for determination of mean arterial pressure. Venous blood aliquots were collected by venipuncture for biochemical evaluation of plasma biomarkers of treatment outcomes and metabolic health status of the rats.

Unpredictable Chronic Mild Stress Protocol: All rats were singly housed, with the control group in a separate quiet room in close proximity to the room used for UCMS treatments. Alternatively, in the UCMS group, rats were randomly exposed to the following mild environmental stressors on multiple occasions throughout each 24 hour period water temperature was $\sim 30^{\circ} \mathrm{C}$ and the room temperature was $\sim 24^{\circ} \mathrm{C}$ :

1. Damp bedding $-10 \mathrm{oz}$. of water was added to each standard cage for the next 3 hours

2. Water - all bedding was removed and $\sim 0.5$ inches of water was added to empty cage for the next 3 hours.

3. Each cage was tilted to 45 degrees with or without bedding for 3 hours

4. Social stress - each rat was switched into a cage of a neighboring rat for 3 hours

5. No bedding lasting for 3 hours or, on two occasions each week, overnight.

6. Succession of light/dark cycles, lasting 30 minutes throughout a 24 hour period

7. Exposure to predator smells (e.g., cat fur) and/or sounds (e.g., cat growling) for 8 hours 
8. After eight weeks, all rats were subjected to a series of behavioral tests to evaluate the outcomes of the UCMS procedures.

Coat Status: This evaluation was done throughout the duration of the UCMS protocol. The total cumulative score was computed by giving an individual score of 0 (clean) or 1 (dirty) to eight body parts (head, neck, dorsal coat, ventral coat, tail, forelimb, hind-limb, and genital region).

Splash Test: This test was used to evaluate acute grooming behavior, defined as cleaning of the fur by licking or scratching. A $10 \%$ sucrose solution was sprayed on the dorsal coat of each rat and grooming activity was recorded for five minutes. The viscosity of the sucrose solution will dirty the coat and induce grooming behavior, with depressive symptoms characterized by an increased latency (idle time between spray and initiation of grooming) and decreased frequency (number of times grooming a particular body part).

Measurements of Vascular Reactivity (Conduit Arteries): Following removal of the resistance arteriole in each rat, the thoracic aorta was removed, rinsed in physiological salt solution, cleared of surrounding tissue, and cut in 2-3 mm ring segments. Each ring was mounted in a myobath chamber between a fixed point and a force transducer (World Precision Instruments), and set to $0.5 \mathrm{~g}$ tension for 45 minutes to equilibrate. The organ baths contained physiological salt solution at $37^{\circ} \mathrm{C}$, and aerated with $95 \% \mathrm{O}_{2}$ and $5 \% \mathrm{CO}_{2}$. Rings were pre-conditioned by treatment with $10^{-7} \mathrm{M}$ phenylephrine for 5 minutes, at which time $10^{-5} \mathrm{M}$ methacholine was added to the bath to assess endothelial integrity. Any ring that failed to demonstrate both a brisk constrictor response to phenylephrine and viable endothelial function was discarded. Subsequently, rings were treated with increasing concentrations of phenylephrine $\left(10^{-10} \mathrm{M}-10^{-4} \mathrm{M}\right)$ to assess constrictor reactivity. For the assessment of dilator reactivity, rings were pre-treated with $10^{-6} \mathrm{M}$ phenylephrine and exposed to increasing concentrations of methacholine $\left(10^{-10} \mathrm{M}-10^{-4} \mathrm{M}\right)$ and sodium nitroprusside $\left(10^{-10} \mathrm{M}-10^{-4} \mathrm{M}\right)$. To assess the roles of nitric oxide, cyclooxygenase and reactive oxidant stress in modulating vascular responses to the agonist treatments, concentration-response curves were also conducted following pretreatment of the rings for 45-60 minutes with L-NAME $\left(10^{-4} \mathrm{M}\right)$, indomethacin $\left(10^{-5} \mathrm{M}\right)$, and TEMPOL $\left(10^{-4} \mathrm{M}\right)$, respectively. 
Biochemical Analyses: Plasma corticosterone was determined using standard EIA kits (Cayman Chemical). Plasma insulin and biomarkers of inflammation were measured by a multiplexed electrochemiluminescence platform (Meso Scale Diagnostics).

Data and Statistical Analyses: Mechanical responses following challenge with methacholine or phenylephrine were fit with the three-parameter logistic equation:

$$
y=\min +\left[\frac{\max -\min }{1+10^{\log E D_{50}-x}}\right]
$$

where $y$ represents the isometric tension, "min" and "max" represent the lower (minimum) and upper (maximum) bounds, respectively, of the change in tone with agonist concentration, $x$ is the logarithm of the agonist concentration and $\log \mathrm{ED}_{50}$ represents the logarithm of the agonist concentration $(x)$ where the response $(y)$ is halfway between the bounds. The use of the three-parameter logistic equation is appropriate for the analysis of sigmoidal concentration-response relationships, as it simultaneously provides estimates of the curve maximum (upper bound), minimum (lower bound) and the dose at which the dependent variable reaches $50 \%$ of maximum $\left(\mathrm{ED}_{50}\right)$. In the case of a dilator response where the initial condition is set to $100 \%$ (the upper bound), the asymptotic minimum (the lower bound from the equation) is reflective of the degree of dilator reactivity for that vessel. If the vessel becomes "more reactive", the lower bound will decrease toward reduced levels, while if the vessel becomes less reactive, the lower bound will increase toward higher values. This situation is reversed for constrictor responses, where the vessel starts at the lower bound, and then goes through a range of increased tensions to reach an upper bound/asymptotic maximum. For the presentation of results, we have focused on the changes in the upper or lower bounds, as we did not determine a consistent or significant change to the $\mathrm{ED}_{50}$ values between relevant experiment groups.

All data are presented as mean \pm SEM. Significant differences between groups were determined using analysis of variance (ANOVA). In all cases, Student-Newman-Keuls post hoc test was used when appropriate and $\mathrm{p}<0.05$ was taken to reflect statistical significance. 


\section{RESULTS}

Table 1 presents data describing the characteristics of LZR and OZR under control and UCMS conditions. After 8 weeks of UCMS, plasma glucose was significantly greater in male and female LZR versus same sex controls, while only elevated in UCMS female OZR versus control female OZR. Inflammatory biomarkers (TNF $\alpha$, IL-6, IL-1 $\beta$ ) were increased in UCMS animals (versus respective controls), and both female-UCMS LZR and OZR exhibited higher levels of all three inflammatory cytokines versus male-UCMS LZR and OZR, respectively. Plasma levels of corticosterone were significantly increased with UCMS in both sexes (OZR and LZR) as compared to relative controls $(\mathrm{p}<0.05)$; corticosterone levels were significantly greater in UCMS-female LZR than that in UCMS-male LZR $(\mathrm{p}<0.05)$.

The impact of the UCMS protocol on depressive behaviors in lean and obese rats is summarized in Figure 1. Throughout 8 weeks of UCMS, the coat status in LZR (Panel A) and OZR (Panel B), undergoing the stress protocol were consistently poorer compared to that in respective control animals $(\mathrm{p}<0.05)$, and the severity of this degradation in coat status was significantly worse in UCMS-female LZR $(\mathrm{p}<0.05)$ versus UCMS male LZR, while no differences were observed between the sexes in OZR.

In response to the sucrose spray, UCMS animals, both LZR and OZR, demonstrated both a less rapid (Panel C) and less frequent (Panel D) grooming response as compared to that exhibited in respective controls $(\mathrm{p}<0.05)$. Significant differences $(\mathrm{p}<0.05)$ between the sexes were observed only in LZR (greater latency and reduced grooming in females) but not OZR following UCMS.

The constrictor responses of aortic rings from lean and obese rats in response to increasing concentrations of phenylephrine are summarized in Figure 2. As compared to responses in aortic rings from control male LZR, rings from UCMS-LZR males exhibited a similar constrictor response to the adrenergic agonist (Panel A). In female LZR, the constrictor response of aortic rings was not significantly different between control and UCMS; however, compared to all groups, constrictor reactivity was least in control females. In OZR, PE-induced constrictor responses were elevated in UCMS animals compared to controls, and this was more pronounced in female OZR $(\mathrm{p}<0.05)$. 
The methacholine-induced dilator responses of aortic rings from control and UCMS-LZR are presented in Figure 3. In comparison to responses from control LZR, the dilation to increasing concentrations of methacholine was significantly reduced in both male and female UCMS LZR, although the severity of this blunted response in aortic rings from UCMS-males (Panel A) was greater $(\mathrm{p}<0.05)$.

Figure 4 presents vasodilator responses in aortic rings of LZR following pre-treatment with various inhibitors. In vessels of control male and female LZR (Panels A and B), pre-treatment with L-NAME resulted in a significant inhibition of methacholine-induced dilator reactivity while pre-treatment with indomethacin showed a less striking, although still significant, attenuation in dilation $(\mathrm{p}<0.05)$. In aortic rings from UCMS-male LZR (Panel C), pre-treatment with L-NAME had minimal effects on further blunting dilator responses while indomethacin almost diminished dilator response to methacholine $(\mathrm{p}<0.05)$. In conduit artery segments from UCMS-female LZR, treatment with L-NAME significantly reduced methacholine-induced dilation $(\mathrm{p}<0.05)$, while responses following treatment with indomethacin was less blunted (Panel D). In all cases, combined treatment with both L-NAME and indomethacin nearly abolished the reactivity of aortic rings to increasing concentrations of methacholine.

The methacholine-induced dilator responses of aortic rings from control and UCMS-OZR are presented in Figure 5. In comparison to responses from control OZR, the dilation to increasing concentrations of methacholine was significantly reduced in both male and female UCMS OZR ( $<<0.05)$, but differences between sexes was not observed. In UCMS male OZR (Panel B) and UCMS female OZR (Panel C) aortic dilator responses were severely attenuated, potentially with a near complete loss of both $\mathrm{NO}$ and $\mathrm{PGI}_{2}$ bioavailability.

Figure 6 summarizes the responses of aortic rings from control and UCMS rats to increasing concentrations of sodium nitroprusside. In both LZR (Panel A) and OZR (Panel B), application of the nitric oxide donor resulted in similar dilator responses between control and UCMS rats of both sexes.

Table 2 presents data comparing the characteristics of control and UCMS OVX LZR and OVX OZR versus their respective intact female animals. OVX was associated with increased weight in both LZR and OZR versus respective intact females $(\mathrm{p}<0.05)$. Plasma glucose and inflammatory biomarkers (TNF $\alpha$, IL-6, IL-1 $\beta$ ) were increased in OVX LZR versus intact females, both with control and UCMS ( $<<0.05)$, and these were 
elevated in UCMS OVX LZR versus control OVX LZR. While plasma glucose and inflammatory biomarkers were significantly elevated in control OVX OZR versus intact control OZR $(p<0.05)$, UCMS did not have an additive effect on increasing these markers in OVX OZR compared to intact UCMS OZR. Triglyceride, cholesterol, LDL, and non LDL were all significantly greater in OVX OZR compared to OVX LZR (data not shown).

Figure 7 shows the impact of the UCMS protocol on depressive behaviors in lean and obese OVX rats versus intact lean and obese females, respectively. In control conditions, coat status in OVX LZR (Panel A) and OVX OZR (Panel B) was not different from respective intact control females. With UCMS, OVX LZR exhibited an attenuated deterioration of coat status compared to intact UCMS LZR ( $\mathrm{p}<0.05)$, and while this pattern was trending in the OZR, the lowered coat score in UCMS OVX OZR (compared to intact UCMS OZR) did not reach significance until the last week of UCMS $(\mathrm{p}<0.05)$.

Loss of estrogen had a substantial effect on vasodilator responses in aortic rings of LZR females, summarized in Figure 8. In control LZR, OVX blunted vasodilator response to methacholine, resulting in a similar degree of vascular dysfunction observed in intact female LZR subjected to UCMS ( $p<0.05$ ), shown in Panel A. The effects of chronic stress further exacerbated impaired vascular reactivity in OVX LZR $(p<0.05)$. When compared to UCMS males, vasodilator response of UCMS OVX females converged at a very similar level, suggesting that lack of estrogen has a role in attenuating sex specific effects of UCMS on vascular function (Panel B). Following pre-treatment with L-NAME, vasodilator responses to methacholine were abolished in both control OVX LZR (Panel C) and UCMS (Panel D). In contrast, indomethacin had less substantial effects on vasodilation in OVX control, while indomethacin (at the highest treatment concentration) minimally improved the blunted response in OVX UCMS LZR compared to non-treated conditions.

Figure 9 presents the dilator responses of aortic rings from control and UCMS OVX OZR in response to increasing concentrations of methacholine. OVX had minimal effects on dilator responses to methacholine in either control or UCMS conditions compared to control and UCMS intact OZR, respectively (Panel A). Treatment with L-NAME completely knocked out dilation in both control (Panel B) and UCMS (Panel C) OVX 
OZR. Inhibition of COX with indomethacin had no effect on vasodilator response in control OVX OZR, while minimal improvement of blunted response (vs. untreated conditions) occurred in OVX UCMS OZR.

Pre-treatment of conduit vessels from UCMS OVX females with TEMPOL (Figure 10) restored vasodilator responses in UCMS OVX LZR to a similar level of untreated OVX control LZR (Panel A). A similar trend was observed in OVX OZR (Panel B), showing TEMPOL improved dilation in UCMS OVX OZR to that of OVX OZR under control conditions. TEMPOL had minimal effects on vasodilator responses of both control OVX LZR and OZR (data not shown).

\section{DISCUSSION}

With a growing body of evidence from clinical and epidemiological studies in support of a substantial sex disparity associated with both the incidence and severity of depression and the development of CVD risk factors, translationally relevant animal models can be used to facilitate further investigations into functional outcomes, underlying mechanisms, and novel therapeutic strategies.

In previous studies, we observed that 8 weeks of UCMS in BALBc/J mice induced behavioral changes that are characteristic of clinical depression (progressive failure to groom; lack of self-stimulatory behavior; and an elevation of circulating cortisol levels) (d'Audiffret et al., 2011; Stanley et al., 2014). In addition, UCMS also significantly impacted vascular reactivity and endothelial function in the gracilis arteriole and aortic rings, as evidenced by alterations to dilator/constrictor balance and impaired endothelial dependent vasodilator response to agonist challenge, as well as promotion of pro inflammatory, pro oxidative conditions within the vasculature. Furthermore, in female mice (versus males), more severe depressive symptoms and greater elevations in plasma cortisol were observed, although the degree of vascular dysfunction was attenuated compared to males; this was suggested to be due to the ability to attenuate the effects of elevated ROS and inflammation on dilator responses. The data suggest that female mice are more susceptible to chronic stress- induced depressive behaviors but have a greater ability to tolerate elevated ROS and systemic inflammatory stress that blunted the subsequent development of vasculopathy compared to males. The purpose of this study was to interrogate the behavioral and physiological effects of chronic exposure to exogenous stressors in male and female rats, under conditions of health as 
well as with pre-existing metabolic syndrome. In addition, these parameters were also measured in ovariectomized (OVX) females in order to interrogate the effects of ovarian hormone deficiency.

Similar to previous reports, female LZR developed more severe depressive like behaviors with UCMS, as evidenced by a deterioration of the coat score and decreased grooming behavior, as well as elevated circulating corticosterone levels, compared to male LZR. Interestingly, although no significant difference between sexes occurred in the OZR, development of depressive like behaviors was not only observed with UCMS, but also in non-stressed animals. This suggests that OZR develop depressive phenotypes in the absence of environmental stress challenges. Unpublished data from our laboratory demonstrate progressive worsening of depressive-like behaviors with age (10 to 19 weeks) as evidenced by deterioration of coat status and elevation of plasma cortisol in OZR males in the absence of additional intervention, and this has been supported in behavioral studies using the OZR (Li et al., 2014). Furthermore, similar models of metabolic syndrome with various genetic mutations of the leptin-receptor demonstrate a pre-disposition towards depressive behaviors (Sharma et al., 2010; Yamada et al., 2011, Guo et al., 2013), indicating a potential role of leptin signaling in the development of depression.

UCMS-induced impairments to vascular vasodilator responses in female LZR were less severe compared to that of males (24\% reduction in vasodilation vs. $40 \%$ ), supporting numerous other reports of superior vascular function in females subjected to stress regimens. In addition, vasodilator responses were further blunted in aortic rings of UCMS female LZR following pre-treatment with L-NAME, while this had minimal effects in UCMS males. Instead, UCMS male LZR showed greater dependence on COX metabolites, like $\mathrm{PGI}_{2}$, likely to maintain dilator responses during compromised NO bioavailability. In contrast to the disparate results observed between male and female LZR, there was no significant difference in vascular reactivity between male and female OZR (50\% reduction in response versus OZR control), indicating a loss of sex-specific vascular protection to UCMS that is associated with significant reductions of both $\mathrm{P} \mathrm{PGI}_{2}$ and $\mathrm{NO}$ bioavailability. While confirming vascular protection to stress in the female LZR, the similarity of impairment between the sexes in the UCMS OZR may be a result of significant loss of sex-specific vasoprotective vascular mechanisms in chronically stressed females with preexisting CVD risk. 
Heightened sensitivity to adrenergic challenge was observed in UCMS male and female OZR via increased contractility of aortic rings to the $\alpha$-adrenergic receptor agonist phenylephrine. Increased sympathetic activity has been etiologically linked not only with depression, but also with the development of some components of metabolic syndrome such as dyslipidemia and hypertension, as well as vascular risk factors including platelet activation and increased pro-inflammatory factors (Goldstein et al., 1983; Golden et al., 1988; Anfossi et al., 1996). Persistent sympathetic over-activity associated with chronic stress/depression and metabolic syndrome may exacerbate vascular dysfunction, as studies in our lab have demonstrated enhanced $\alpha$-adrenergic vasoconstriction in arterioles of OZR, resulting in impaired blood flow to skeletal muscle and the microvessel networks that negatively affect tissue perfusion (Frisbee, 2002).

It is increasingly evident that chronic activation of pro-inflammatory pathways may be partly responsible for obesity-induced metabolic disorders (Wellen et al., 2005). Biomarkers of inflammation (TNF- $\alpha$, IL-6, IL-1 $\beta$ ) were greater in UCMS animals relative to their respective controls, with greatest elevation in obese animals. Comorbid metabolic syndrome and depression seemed to mitigate the protective vascular mechanisms to UCMS observed in lean females; this may be partially attributed to the severity of the chronic inflammatory microenvironment in UCMS female OZR. Advanced metabolic disease coupled with chronic stress may simply overwhelm the stress response at the most basic mechanistic levels, resulting in chronic, systemic inflammation and unmitigated oxidative stress associated with the severe endothelial dysfunction demonstrated in UCMS OZR in this study.

\section{$\underline{O V X}$ and UCMS}

The effects of female sex hormone deficiency on behavioral outcomes and vascular dysfunction induced by UCMS were investigated in lean and obese animals. Estrogen in particular has been implicated in a range of antioxidant vasculoprotective functions; OVX was therefore performed on female animals to eliminate the major source of estrogen production. Following UCMS, both OVX OZR and LZR developed depressive symptoms (i.e. worsened coat score and decreased grooming), though these impaired behaviors were less severe than intact UCMS female OZR and LZR, respectively, with no change in control conditions. Loss of estrogen may impact the severity of developed behavioral responses under conditions of induced chronic stress that are attenuated or less striking in non-stressed conditions. As women are twice as likely to develop depression, specifically during 
times of intense hormone fluctuations (Steiner et al., 2003), it may be that lack of constant estrogen cycling in OVX animals confers a long term effect on behavior that attenuates depressive symptoms. However, behavioral effects mediated by estrogen have demonstrated controversial findings in both animal and human studies.

Local vascular factors, such as NO, are important regulators of peripheral resistance and endothelium and VSM function. NO bioavailability is essential for regulation of blood pressure and local blood flow, in addition to its inhibitory actions on platelet aggregation, thrombus formation, and leukocyte adhesion. Increasing evidence suggests that the vasculoprotective effects of estrogen are due to enhancement of vascular NO bioavailability as well as its functional role in antioxidant defense and anti-inflammatory mechanisms in multiple different tissues (Thompson et al., 2000). In this study, L-NAME completely abrogated vascular reactivity in all OVX animals, indicating severely compromised NO bioavailability in estrogen deficient animals. In addition, estrogen may also influence production of $\mathrm{PGI}_{2}$ via up-regulation of the $\mathrm{COX}$ pathway, specifically $\mathrm{COX}-1$ and prostacyclin synthase (Sherman et al., 2002), implicating a role of estrogen in regulating vasodilator and vasoconstrictor balance of arachidonic acid metabolites. For example, conditions of estrogen deficiency in women (i.e., aging, hypertension, and diabetes) are associated with a shift in favor of COX-dependent vasoconstrictors over vasodilators. Alterations in COX metabolism have been associated with differential effects on methacholinestimulated endothelium-dependent dilation between males and females (Li et al., 2008), specifically during conditions of chronic stress/depression (Stanley et al., 2014). In contrast to intact lean and obese females, minimal effects were observed following inhibition of COX with indomethacin in OVX animals, indicating lack of $\mathrm{PGI}_{2}$ in vasodilator responses to methacholine and/or altered vasoconstrictor mediated activity in OVX state. Together, alterations in NO and $\mathrm{TxA}_{2}$ bioavailability in the vascular network of OZR have been demonstrated to contribute to significant changes in the severity of adrenergic constriction, myogenic activation, and the integration of these components on regulating vascular tone (Frisbee et al., 2002), which is potentiated under comorbid depression.

Loss of estrogen alone was sufficient to attenuate vasodilator response to methacholine challenge in control females, suggesting that OVX blunts vascular reactivity under non-stressed conditions. Following UCMS, OVX further blunted vasodilator responses in LZR, so that the level of vascular dysfunction was comparable to that of UCMS male LZR. It appears that loss of estrogen in lean females is associated with failure to confer 
protection against chronic stress- induced vasculopathy, resulting in similar vascular outcomes to males. A similar relationship in vascular dysfunction between intact lean and obese females mentioned previously was sustained in OVX conditions, but just shifted to a higher degree of dysfunction with estrogen loss. Furthermore, increased inflammatory markers (TNF- $\alpha$, IL-6, IL-1 $1 \beta$ ) in OVX females may be due to loss of estrogenic anti-inflammatory action, which was exacerbated with UCMS. Hyperglycemia and increased body mass may have additional effects in promoting oxidative conditions (Perez-Torres, et al., 2008), further impairing NO bioavailability and promoting inflammation. Comorbid depressive symptoms and metabolic syndrome induce an inflammatory environment that overwhelms compensatory mechanisms and results in severe vascular impairments, adverse outcomes that are exacerbated by removal of estrogen by OVX in this study.

The effects of OVX and chronic stress appear to impact vascular reactivity differently in lean versus obese females. For example, in LZR, the severity of impaired vasodilator responses was more associated with OVX, while OVX appeared to have less of an impact on vascular reactivity in OZR compared to that of chronic stress (with UCMS). This was in parallel to inflammatory biomarker levels, which were substantially greater in OVX LZR versus intact LZR, while comparable between OVX OZR and UCMS OZR. While beyond the scope of this study, female OZR have been reported to be infertile as a result of reduced levels of sex hormones, and such attenuated activity may in part explain the smaller impact of OVX on vascular function in OZR. It is possible that restoring functional activity of ovarian hormones with exogenous administration of estrogen may have a more pronounced effect on vascular function in female OZR. In addition, estrogen has a major role in body weight homeostasis, lipid metabolism, glycemic control, and total and central abdominal adiposity and this has been associated with variable effects in high-risk CVD individuals (Mallei et al., 2006; Perez-Torres et al., 2008). Results presented here show that OVX OZR had significantly increased levels of triglycerides, cholesterol, and low density lipoprotein levels compared to OVX LZR, which could be major sources of oxidative stress and inflammation in this CV model. However, another study using the OZR demonstrated unfavorable effects of estrogen on triglyceride and cholesterol profiles that exacerbated metabolic abnormalities associated with underlying metabolic syndrome (Gades et al., 1998). Taken together, it is possible that the beneficial effects of 
estrogen on vascular health are overwhelmed by the pro-inflammatory, oxidative environment in OZR, thereby contributing to similar outcomes in OVX and intact OZR females.

These results support the role of inflammation, oxidative stress, and decreased NO bioavailability as important mechanisms contributing to chronic stress-induced vascular dysfunction in estrogen-deficient lean and obese females. Understanding the variables associated with the relationship between obesity and depression is essential given the increased health risks associated with comorbidity and the potential implications for sex specific prevention and treatment plans of comorbid obesity and depression.

\section{ACKNOWLEDGEMENTS}

The authors gratefully acknowledge the Office of Laboratory Animal Research at West Virginia University for their expertise and assistance during the performance of these procedures. Additionally, we also acknowledge the support provided through Center for Cardiovascular and Respiratory Sciences at the West Virginia University Health Sciences Center.

\section{SOURCES OF RESEARCH FUNDING}

This study was supported by the American Heart Association (IRG 14330015, PRE 16850005, EIA 0740129N), and the National Institutes of Health (RR 2865AR; P20 RR 016477). 


\section{REFERENCES}

1. Krishnan V, Nestler EJ. Linking molecules to mood: new insight into the biology of depression. Am J Psychiatry, 2010; 167(11), 1305-1320.

2. Plante GE. Depression and cardiovascular disease: a reciprocal relationship. Metabolism, 2005; 54(5 Suppl 1), 45-48.

3. Sareen J, Jacobi F, Cox BJ, Belik SL, Clara I, Stein MB. Disability and poor quality of life associated with comorbid anxiety disorders and physical conditions. Arch Intern Med. 2006; 166(19):2109-16.

4. Kuehner C. Gender differences in unipolar depression: an update of epidemiological findings and possible explanations. Acta Psychiatr Scand. 2003;108(3):163-74.

5. Daniel JM, Hulst JL, Lee CD. Role of hippocampal M2 muscarinic receptors in the estrogen-induced enhancement of working memory. Neuroscience. 2005;132(1):57-64.

6. Talboom JS, Williams BJ, Baxley ER, West SG, Bimonte-Nelson HA. Higher levels of estradiol replacement correlate with better spatial memory in surgically menopausal young and middle-aged rats. Neurobiol Learn Mem. 2008; 90(1):155-63.

7. MacRitchie AN, Jun SS, Chen Z, German Z, Yuhanna IS, Sherman TS, Shaul PW. Estrogen upregulates endothelial nitric oxide synthase gene expression in fetal pulmonary artery endothelium. Circ Res. 1997;81(3):355-62.

8. Kamper EF, Chatzigeorgiou A, Tsimpoukidi O, Kamper M, Dalla C, Pitychoutis PM, Papadopoulou-Daifoti Z. Sex differences in oxidant/antioxidant balance under a chronic mild stress regime. Physiol Behav. 2009;98(1-2):215-22.

9. Wang D, Wang C, Wu X, Zheng W, Sandberg K, Ji H, Welch WJ, Wilcox CS. Endothelial dysfunction and enhanced contractility in microvessels from ovariectomized rats: roles of oxidative stress and perivascular adipose tissue. Hypertension 2014; 63:1063-1069

10. Wellen GS. Inflammation, stress, and diabetes J. Clin. Invest. 2005; 115:1111-1119 
11. Sherman TS, Chambliss KL, Gibson LL, Pace MC, Mendelsohn ME, Pfister SL, and Shaul PW. Estrogen acutely activates prostacyclin synthesis in ovine fetal pulmonary artery endothelium. Am J Respir Cell Mol Biol 2002; 26:610-616.

12. Thompson LP, Pinkas G, and Weiner CP. Chronic 17ß-estradiol replacement increases nitric oxide-mediated vasodilation of guinea pig coronary microcirculation. Circulation 2000; 102:445-451.

13. Dalla, C., Antoniou, K., Drossopoulou, G., Xagoraris, M., Kokras, N., Sfikakis, A., \& Papadopoulou-Daifoti, Z. Chronic mild stress impact: are females more vulnerable? Neuroscience. 2005; 135(3), 703-714.

14. Grippo AJ, Sullivan NR, Damjanoska KJ, Crane JW, Carrasco GA, Shi J, Van de Kar LD. Chronic mild stress induces behavioral and physiological changes, and may alter serotonin 1A receptor function, in male and cycling female rats. Psychopharmacology (Berl), 2005; 179(4):769-780.

15. Rupnow HL, Phernetton TM, Shaw CE, Modrick ML, Bird IM, Magness RR. Endothelial vasodilator production by uterine and systemic arteries. VII. Estrogen and progesterone effects on eNOS. Am J Physiol Heart Circ Physiol, 2001; 280(4):H1699-1705.

16. Willner P. Validity, reliability and utility of the chronic mild stress model of depression: a 10-year review and evaluation. Psychopharmacology (Berl). 1997; 134(4):319-329.

17. Everson-Rose SA., TT Lewis. Psychosocial factors and cardiovascular diseases. Annual Review of Public Health. 2005; 26:469-500

18. Li S, Zhai X, Rong P, McCabe MF, Wang X, Zhao J, Ben H, Wang S. Therapeutic effect of vagus nerve stimulation on depressive-like behavior, hyperglycemia and insulin receptor expression in Zucker fatty rats. PLoS One. 2014; 9(11)

19. Lang UE, Borgwardt S. Molecular mechanisms of depression: perspectives on new treatment strategies. Cell Physiol Biochem 2013; 31:761-777

20. Rustad JK, Musselman DL, Nemeroff CB. The relationship of depression and diabetes: Pathophysiological and treatment implications. Psychoneuroendocrinology.2011; 36:1276-1286

21. Goldstein DS. Plasma catecholamines and essential hypertension: an analytical review. Hypertension 1983; 5: $86-99$. 
22. Barden N. Implication of the hypothalamic-pituitary-adrenal axis in the physiopathology of depression. J Psychiatry Neurosci, 2004; 29(3):185-193.

23. Bayramgurler D, Karson A, Yazir Y, Celikyurt IK, Kurnaz S, Utkan T. The effect of etanercept on aortic nitric oxide-dependent vasorelaxation in an unpredictable chronic, mild stress model of depression in rats. Eur J Pharmacol, 2013; 710(1-3):67-72.

24. Beck KD, Luine VN. Sex differences in behavioral and neurochemical profiles after chronic stress: role of housing conditions. Physiol Behav, 2002; 75(5):661-673.

25. Golden RN, Markey SP, Risby ED, Rudorfer MV,Cowdry RW, Potter WZ. Antidepressants reduce whole body norepinephrine turnover while enhancing 6-hydroxymelatonin output. Arch Gen Psychiatry 1988; 45: $150-154$.

26. Anfossi G, Trovati M. Role of catecholamines in platelet function: pathophysiological and clinical significance. Eur J Clin Invest 1996; 26:353-370.

27. Frisbee JC. Regulation of in situ skeletal muscle arteriolar tone: interactions between two parameters. Microcirculation 2002; 9:443-462.

28. Steiner M, Dunn E, Born L.Hormones and mood: from menarche to menopause and beyond. J Affect Disord. 2003; 74(1):67-83.

29. Isingrini E, Belzung C, Freslon JL, Machet MC, Camus V. Fluoxetine effect on aortic nitric oxide-dependent vasorelaxation in the unpredictable chronic mild stress model of depression in mice. Psychosom Med, 2012; 74(1):63-72.

30. Patel V, Garrison P, de Jesus Mari J, Minas H, Prince M, Saxena S. Advisory group of the Movement for Global Mental, H. The Lancet's series on global mental health: 1 year on. Lancet, 2008; 372(9646):13541357.

31. Whiteford HA, Degenhardt L, Rehm J, Baxter AJ, Ferrari AJ, Erskine HE,Vos T. Global burden of disease attributable to mental and substance use disorders: findings from the Global Burden of Disease Study 2010. Lancet, 382(9904), 1575-1586. 
32. Li M, Kuo L, Stallone JN. Estrogen potentiates constrictor prostanoid function in female rat aorta by upregulation of cyclooxygenase-2 and thromboxane pathway expressionAm J Physiol Heart Circ Physiol. 2008; 294(6):H2444-55.

33. Mallei S, Mercuri A, Zucchelli GC, Vassalle C. Estrogen therapy effects on different vasoactive factors in recent postmenopausal healthy women. Int I Cardiol. 2006; 107:194-199.

34. Gades MD, Stern JS, van Goor H, et al., Estrogen accel- erates the development of renal disease in female obese Zuckerrats. Kidney Int. 1998; 53:130-135

35. Sharma AN, Elased KM, Garrett TL, Lucot JB. Neurobehavioral deficits in $\mathrm{db} / \mathrm{db}$ diabetic mice. Physiol Behav. 2010; 101(3):381-8.

36. Yamada N, Katsuura G, Ochi Y, Ebihara K, Kusakabe T, Hosoda K, Nakao K. Impaired CNS leptin action is implicated in depression associated with obesity Endocrinology. 2011; 152(7):2634-43.

37. Guo M, Huang TY, Garza JC, Chua SC, Lu XY. Selective deletion of leptin receptors in adults hippocampus induces depression-related behaviours. Int J Neuropsychopharmacol. 2013; 16(4):857-67.

38. Pérez-Torres I, ElHafidi M, InfanteO, BañosG.Effects of sex Hormone levels on aortic vascular reactivity and variables associated with the metabolic syndrome in sucrose-fed female rats. Can J Physiol Pharmacol. 2008; $86(1-2): 25-35$. 


\section{FIGURE LEGENDS}

Figure 1. Depressive symptoms following eight weeks of the UCMS protocol in male and female LZR and OZR. Data are presented for LZR coat status (Panel A), OZR coat status (Panel B), the latency (Panel C) and frequency (Panel D) of facial grooming following a 10\% sucrose solution spray in LZR and OZR. Control male LZR, OZR $\mathrm{n}=8$; Control female LZR, OZR n=8; UCMS male LZR, OZR n=8, UCMS female LZR, OZR $n=8 . \quad * \mathrm{p}<0.05$ versus controls in the same sex group (LZR or OZR); $\uparrow \mathrm{p}<0.05$ versus UCMS-males of the same group (LZR or OZR).

Figure 2. Constrictor responses of aortic rings to increasing concentrations of phenylephrine from rats under control conditions and after eight weeks of UCMS. Data are presented for LZR (Panel A) and OZR (Panel B). Control male LZR, OZR n=8; Control female LZR, OZR n=8; UCMS male LZR, OZR n=8, UCMS female LZR, OZR $n=8 . * p<0.05$ versus controls in the same sex group (LZR or OZR).

Figure 3. Dilator responses of aortic rings to increasing concentrations of methacholine from LZR under control conditions and after eight weeks of UCMS. Panel A presents data under untreated conditions. Control male LZR $\mathrm{n}=8$; Control female LZR $\mathrm{n}=8$; UCMS male LZR $\mathrm{n}=8$, UCMS female LZR $\mathrm{n}=8$. ${ }^{*} \mathrm{p}<0.05$ versus dilator responses in same sex control; $\uparrow \mathrm{p}<0.05$ versus UCMS-male LZR.

Figure 4. Dilator responses of aortic rings to increasing concentrations of methacholine from LZR under control conditions and after eight weeks of UCMS. Dilator responses from control male (Panel A) and control female (Panel B)control LZR are shown following pre-treatment with L-NAME, indomethacin or combination. Panels C and D present data describing dilator responses from male and female UCMS LZR, respectively, following pretreatment with L-NAME, indomethacin or combination. Control male LZR n=8; Control female LZR n=8; UCMS male LZR $n=8$, UCMS female LZR $n=8 .{ }^{*} \mathrm{p}<0.05$ versus untreated aortic rings; $\uparrow \mathrm{p}<0.05$ versus UCMSmale LZR. 
Figure 5. Dilator responses of aortic rings to increasing concentrations of methacholine from OZR under control conditions and after eight weeks of UCMS. Panel A presents data from untreated vessels in male and female OZR. Panel B presents data describing dilator responses from male UCMS OZR, following pre-treatment with LNAME, indomethacin or combination. Panel C presents data describing dilator responses from female UCMS OZR, following pre-treatment with L-NAME, indomethacin or combination. Control male OZR n=8; Control female OZR n=8; UCMS male OZR n=8, UCMS female OZR $n=8$. *p<0.05 versus responses in control/untreated conditions.

Figure 6. Dilator responses of aortic rings to increasing concentrations of sodium nitroprusside from rats under control conditions and after eight weeks of UCMS. Panel A presents data from LZR. Panel B presents data from OZR. Control male LZR, OZR n=8; Control female LZR, OZR n=8; UCMS male LZR, OZR n=8, UCMS female LZR, OZR n=8.

Figure 7. Depressive symptoms following eight weeks of the UCMS protocol (versus respective control) in OVX LZR (versus intact female LZR) and OVX OZR (versus intact female OZR). Data are presented for LZR coat status (Panel A) and OZR coat status (Panel B), in both OVX and intact females. Control female LZR n=8; Control OVX LZR n=8; UCMS female LZR n=8, UCMS OVX LZR n=8, Control female OZR n=8; Control OVX OZR n=8; UCMS female OZR n=8, UCMS OVX OZR $n=8 .{ }^{*} p<0.05$ versus intact control females; $\dagger$ $\mathrm{p}<0.05$ versus intact females of the same respective groups (control or UCMS).

Figure 8. Dilator responses of aortic rings to increasing concentrations of methacholine from OVX LZR under control conditions and after eight weeks of UCMS. Panels A present data describing dilator responses under untreated conditions in control and UCMS OVX LZR versus intact control and UCMS female LZR. Panel B compares the dilator responses to methacholine in UCMS male LZR versus UCMS OVX LZR. Panel C shows the dilator responses from OVX control LZR, following pre-treatment with L-NAME, indomethacin or combination. Panel D present data describing dilator responses from OVX UCMS LZR, following pre-treatment with LNAME, indomethacin or combination. Control female LZR n=8; Control OVX LZR n=8; UCMS female LZR $\mathrm{n}=8$, UCMS OVX LZR n=8. *p<0.05 versus dilator response in untreated vessel of intact control LZR; $\uparrow \mathrm{p}<0.05$ 
versus dilator responses in control OVX LZR; $\$ \mathrm{p}<0.05$ versus dilator responses in untreated vessels of the same group (control or UCMS).

Figure 9. Dilator responses of aortic rings to increasing concentrations of methacholine from OVX OZR under control conditions and after eight weeks of UCMS. Panels A present data describing dilator responses under untreated conditions in control and UCMS OVX OZR versus intact control and UCMS female OZR. Panel B presents data describing dilator responses from control OVX OZR, following pre-treatment with L-NAME, indomethacin or combination. Panel $\mathrm{C}$ present data describing dilator responses from UCMS OVX OZR, following pre-treatment with L-NAME, indomethacin or combination. Control female OZR n=8; Control OVX OZR n=8; UCMS female OZR n=8, UCMS OVX OZR n=8. *p<0.05 versus responses in intact control OZR; † $\mathrm{p}<0.05$ versus dilator responses in untreated vessels of the same group (control or UCMS).

Figure 10. Dilator responses to increasing concentrations of methacholine in aortic rings of UCMS OVX LZR and OZR following pretreatment with TEMPOL. Panel A presents data from UCMS OVX LZR under untreated conditions and following pre-treatment with TEMPOL, Panel B presents data from UCMS OVX OZR under untreated conditions and following pre-treatment with TEMPOL, Control OVX LZR n=8; Control OVX OZR $\mathrm{n}=8$; UCMS OVX LZR $\mathrm{n}=8$, UCMS OVX OZR $n=8$. * $\mathrm{p}<0.05$ versus dilator response in the respective untreated vessel of OVX females (LZR or OZR). 
Table 1. Baseline characteristics of male and female LZR and OZR within the present study. ${ }^{*} \mathrm{p}<0.05$ versus control animals of same group (LZR or OZR).

\begin{tabular}{|c|c|c|c|c|c|c|c|c|}
\hline & $\begin{array}{c}\text { LZR-M } \\
\text { Control } \\
(n=8)\end{array}$ & $\begin{array}{c}\text { LZR-F } \\
\text { Control } \\
(n=8)\end{array}$ & $\begin{array}{c}\text { LZR-M } \\
\text { UCMS } \\
(n=8)\end{array}$ & $\begin{array}{c}\text { LZR-F } \\
\text { UCMS } \\
(n=8)\end{array}$ & $\begin{array}{c}\text { OZR-M } \\
\text { Control } \\
(n=8)\end{array}$ & $\begin{array}{c}\text { OZR-F } \\
\text { Control } \\
(n=8)\end{array}$ & $\begin{array}{c}\text { OZR-M } \\
\text { UCMS } \\
(n=8)\end{array}$ & $\begin{array}{c}\text { OZR-F } \\
\text { UCMS } \\
(n=8)\end{array}$ \\
\hline Weight (g) & $391 \pm 4.1$ & $229 \pm 5.6$ & $422 \pm 14$ & $244 \pm 2.8$ & $596 \pm 48$ & $572 \pm 22$ & $661 \pm 14$ & $535 \pm 11$ \\
\hline MAP (mmHg) & $106 \pm 0.7$ & $109 \pm 1.5$ & $110 \pm 2.6$ & $113 \pm 1.2$ & $132 \pm 12$ & $132 \pm 0.8$ & $137 \pm 4.1$ & $142 \pm 3.7 *$ \\
\hline Insulin (ng/mL) & $2.4 \pm 0.7$ & $1.0 \pm 0.7$ & $1.7 \pm 0.6$ & $1.8 \pm 0.3$ & $18 \pm 9.6$ & $6.1 \pm 1.9$ & $7.3 \pm 3.1$ & $4.8 \pm 0.9$ \\
\hline Leptin (ng/mL) & $25 \pm 5.4$ & $17 \pm 2.3$ & $15 \pm 2.3$ & $22 \pm 5.4$ & $102 \pm 32$ & $79 \pm 5.0$ & $109 \pm 18$ & $102 \pm 10$ \\
\hline $\mathrm{IL}-1 \beta(\mathrm{pg} / \mathrm{mL})$ & $6.3 \pm 3.2$ & $6.2 \pm 1.2$ & $10.6 \pm 2.0$ & $13 \pm 2.4^{*}$ & $5.1 \pm 1.2$ & $9.0 \pm 2.3$ & $13 \pm 3.4^{*}$ & $16 \pm 5.8$ \\
\hline $\begin{array}{l}\text { Corticosterone } \\
\quad(\mathrm{ng} / \mathrm{mL})\end{array}$ & $87 \pm 3.5$ & $176 \pm 26$ & $105 \pm 12 *$ & $319 \pm 49 * \dagger$ & $111 \pm 15$ & $146 \pm 28.5$ & $289 \pm 48 *$ & $360 \pm 43^{*}$ \\
\hline
\end{tabular}


Table 2. Baseline characteristics of OVX females compared to their respective intact females. *p $<0.05$ versus OVX control of same group (LZR or $\mathrm{OZR}), \uparrow \mathrm{p}<0.05$ versus intact females of similar group (LZR or OZR) and condition (control or UCMS).

\begin{tabular}{|c|c|c|c|c|c|c|c|c|}
\hline & $\begin{array}{c}\text { LZR-F } \\
\text { Control } \\
(n=8)\end{array}$ & $\begin{array}{c}\mathrm{LZR}-\mathrm{F} \\
\mathrm{UCMS} \\
(\mathrm{n}=8)\end{array}$ & $\begin{array}{c}\text { OVX-LZR } \\
\text { Control } \\
(\mathrm{n}=8)\end{array}$ & $\begin{array}{c}\text { OVX-LZR } \\
\text { UCMS } \\
(n=8)\end{array}$ & $\begin{array}{c}\text { OZR-F } \\
\text { Control } \\
(n=8)\end{array}$ & $\begin{array}{c}\text { OZR-F } \\
\text { UCMS } \\
(n=8)\end{array}$ & $\begin{array}{c}\text { OVX-OZR } \\
\text { Control } \\
(n=8)\end{array}$ & $\begin{array}{c}\text { OVX-OZR } \\
\text { UCMS } \\
(n=8)\end{array}$ \\
\hline Weight (g) & $229 \pm 5.6$ & $244 \pm 2.8$ & $302 \pm 7.4 \dagger$ & $285 \pm 4.8 \dagger$ & $572 \pm 22$ & $535 \pm 11$ & $593 \pm 15$ & $585 \pm 9.7 \dagger$ \\
\hline $\begin{array}{c}\mathrm{MAP} \\
(\mathrm{mmHg})\end{array}$ & $108 \pm 1.5$ & $114 \pm 1.2$ & $107 \pm 4.3$ & $117 \pm 3.8$ & $132 \pm 0.8$ & $140 \pm 3.7$ & $142 \pm 6.1$ & $145 \pm 6.6$ \\
\hline $\begin{array}{l}\text { Glucose } \\
\text { (mg/dL) }\end{array}$ & $114 \pm 6.5$ & $136 \pm 8.5$ & $190 \pm 12 \dagger$ & $205 \pm 16 \dagger$ & $131 \pm 9.2$ & $158 \pm 8.2$ & $170 \pm 11 \dagger$ & $157 \pm 10$ \\
\hline $\begin{array}{c}\mathrm{TNF} \alpha \\
(\mathrm{pg} / \mathrm{mL})\end{array}$ & $2.9 \pm 0.9$ & $6.4 \pm 1.1$ & $7.0 \pm 0.5 \dagger$ & $7.1 \pm 0.4$ & $5.7 \pm 0.7$ & $8.8 \pm 1.3$ & $9.1 \pm 1.0 \dagger$ & $7.8 \pm 0.3$ \\
\hline IL-6 (pg/mL) & $2.1 \pm 1.1$ & $30 \pm 13$ & $50 \pm 7.9 \dagger$ & $75 \pm 11 * \dagger$ & $19 \pm 1.1$ & $77 \pm 9.3$ & $75 \pm 13 \dagger$ & $57 \pm 12$ \\
\hline $\begin{array}{c}\mathrm{IL}-1 \beta \\
(\mathrm{pg} / \mathrm{mL})\end{array}$ & $6.2 \pm 1.2$ & $13 \pm 2.4$ & $12 \pm 4.6$ & $15 \pm 1.4$ & $9 \pm 1.3$ & $14 \pm 5.5$ & $15 \pm 2.0 \dagger$ & $14 \pm 3.2$ \\
\hline
\end{tabular}



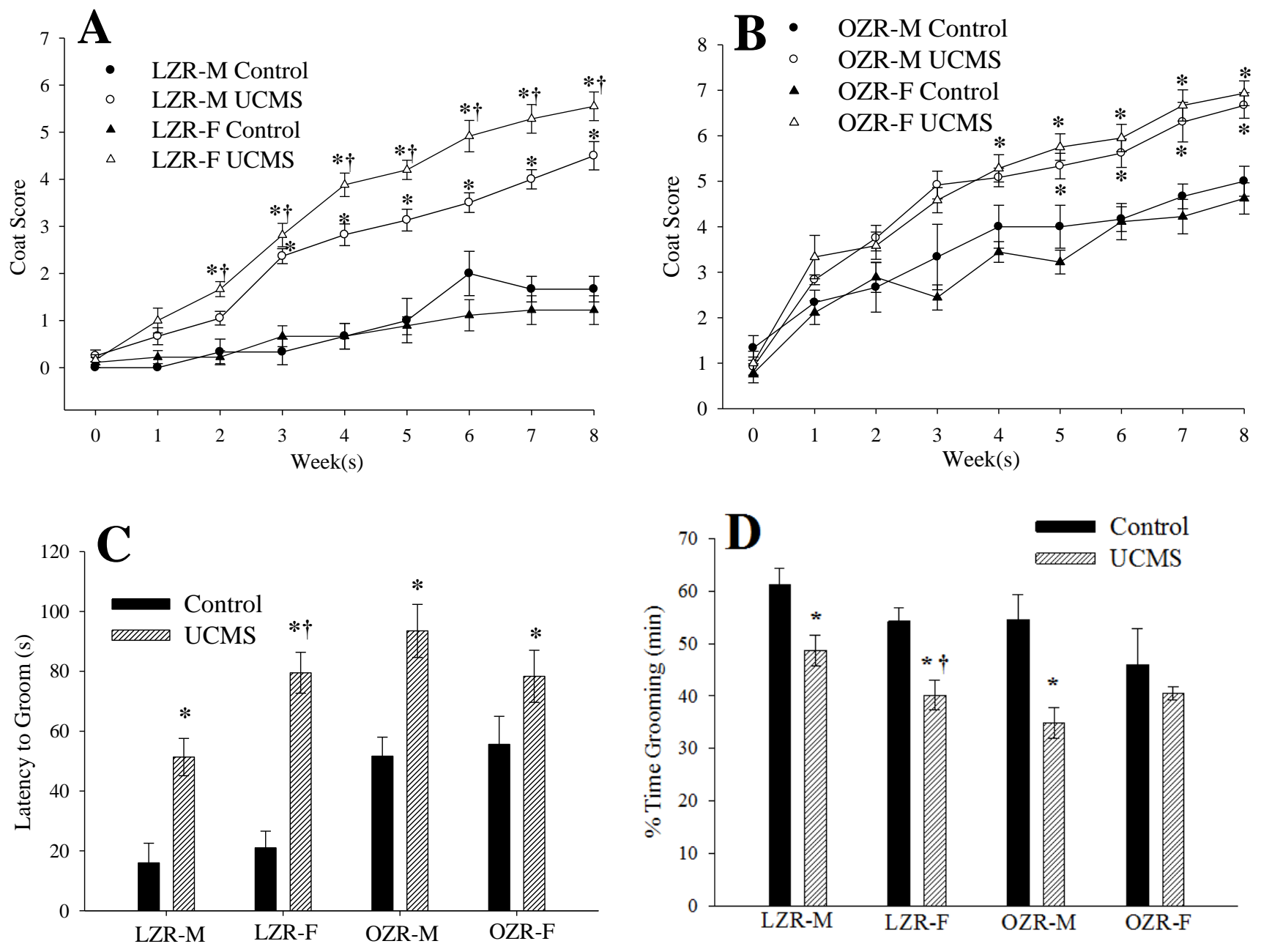

Figure 1

Stanley et al. 

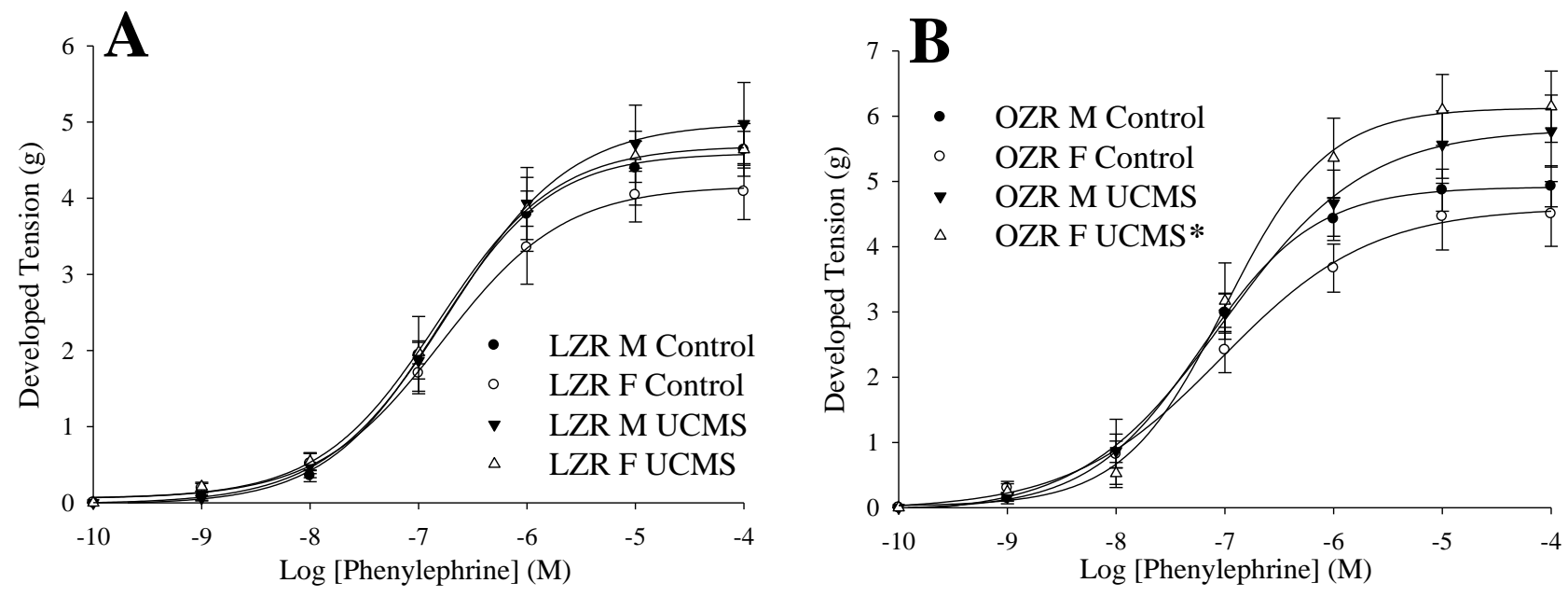

Figure 2

Stanley et al. 


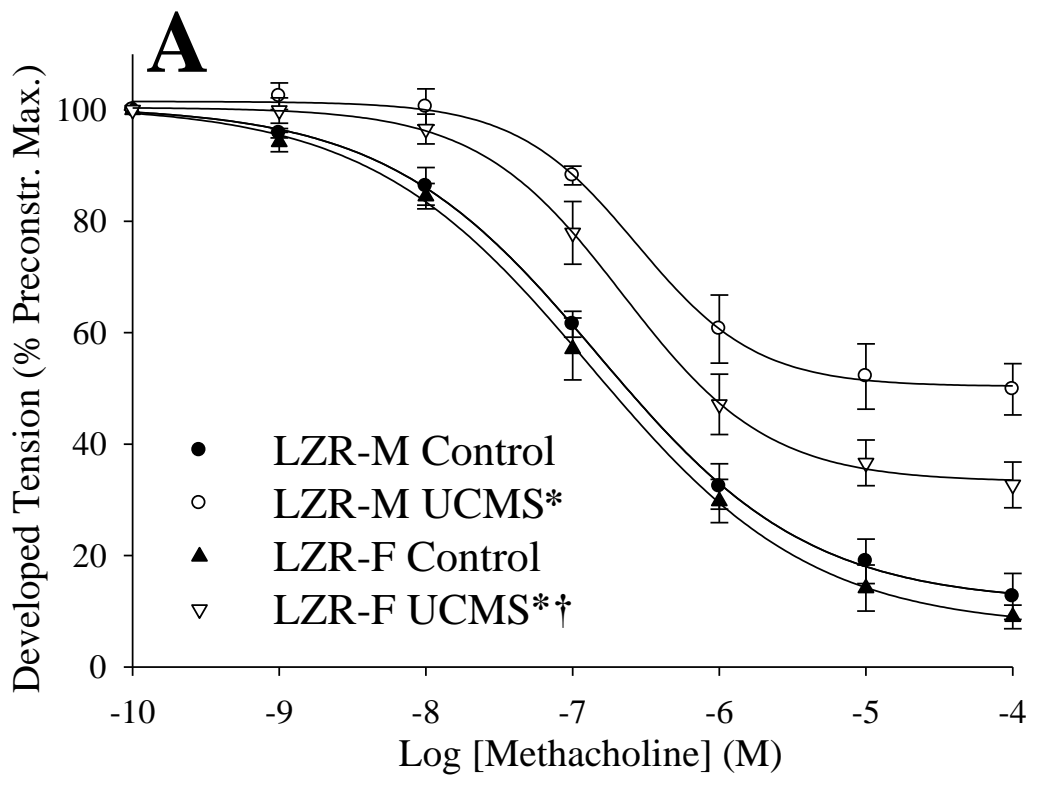

Figure 3

Stanley et al. 

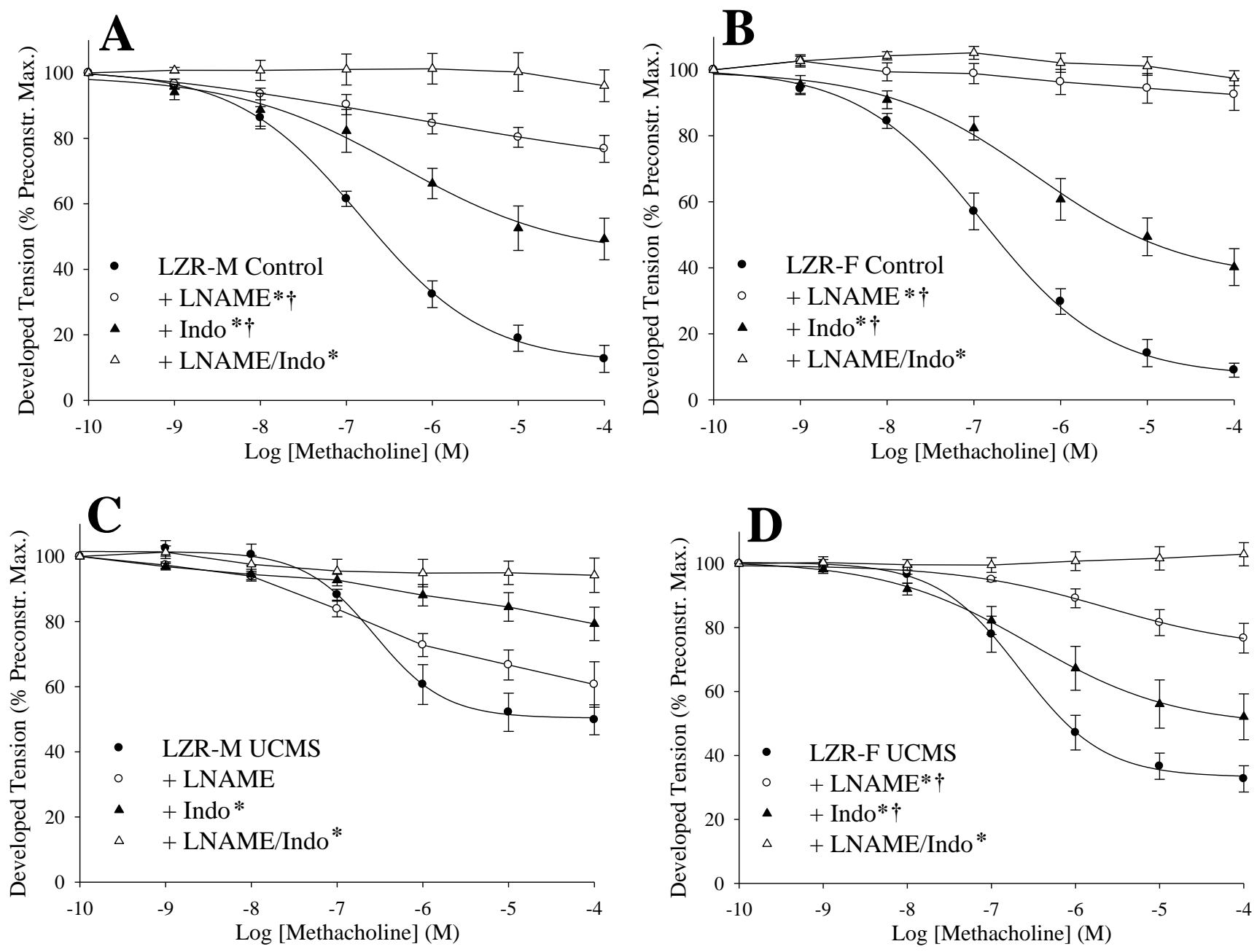

Figure 4

Stanley et al. 

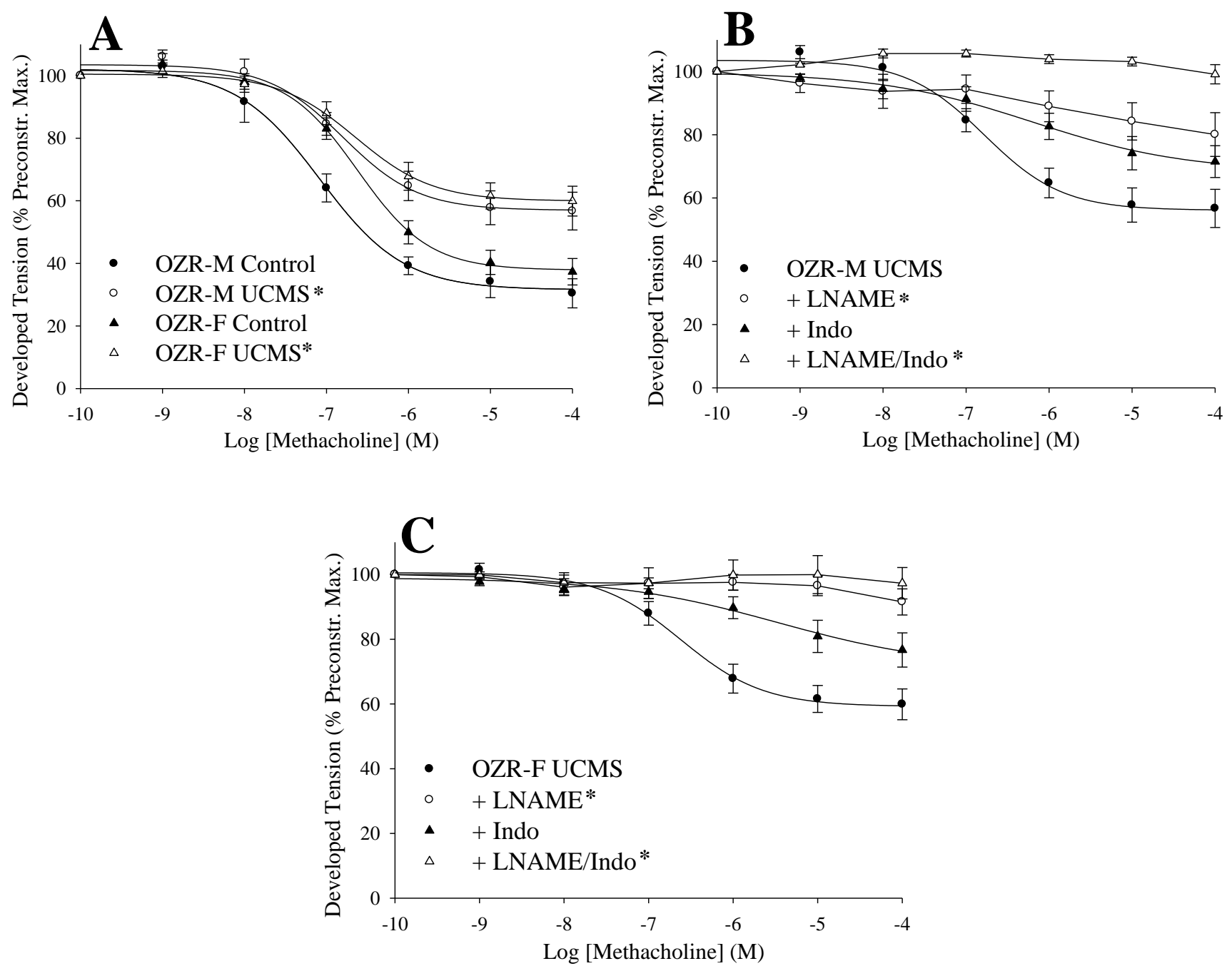

Figure 5

Stanley et al. 

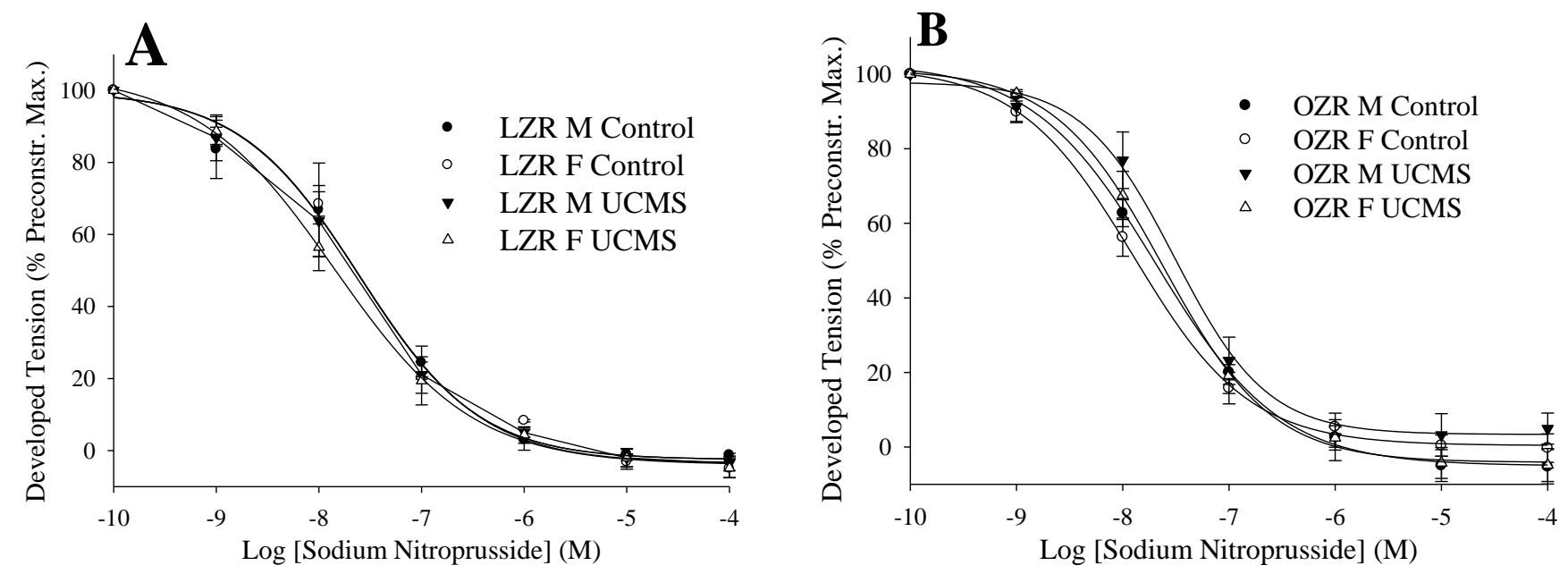

Figure 6

Stanley et al. 

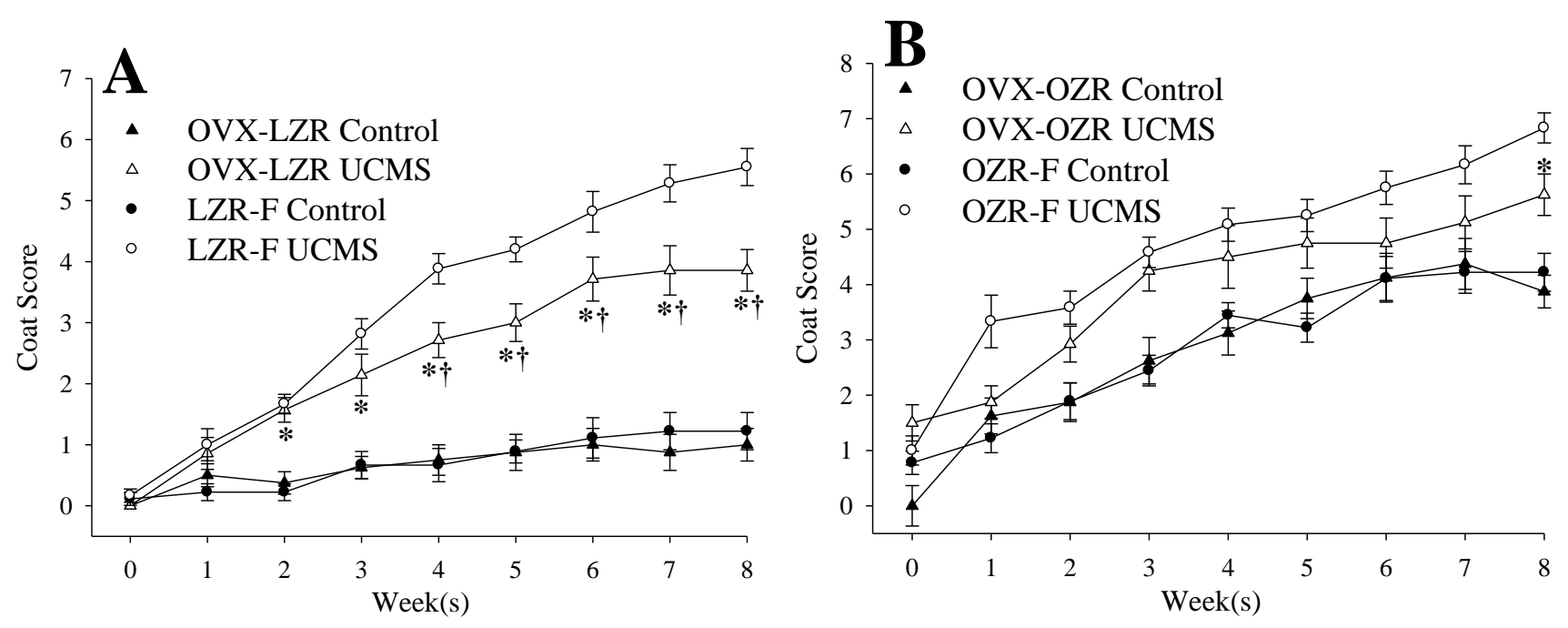

Figure 7

Stanley et al. 

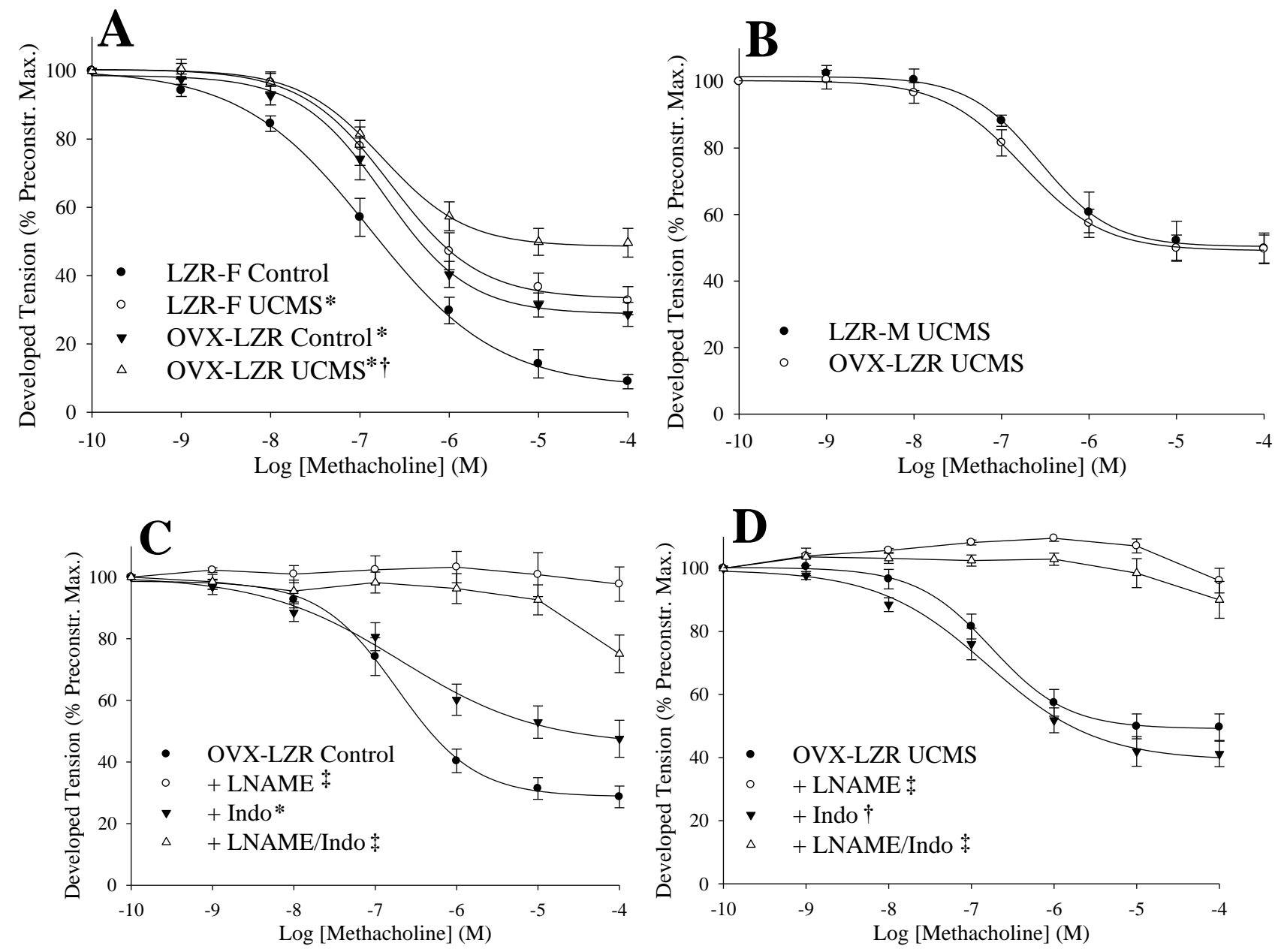

Figure 8

Stanley et al. 

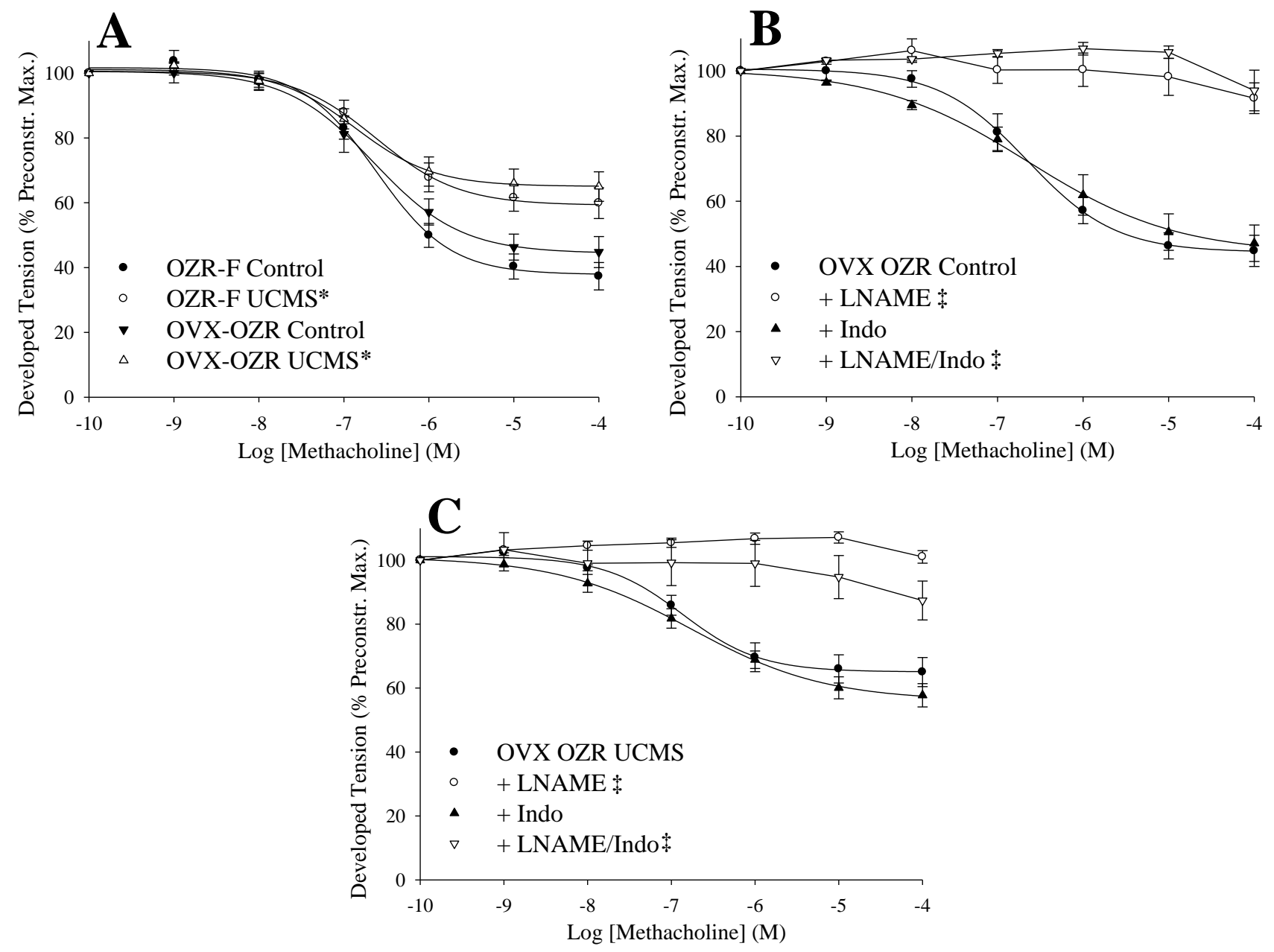

Figure 9

Stanley et al. 

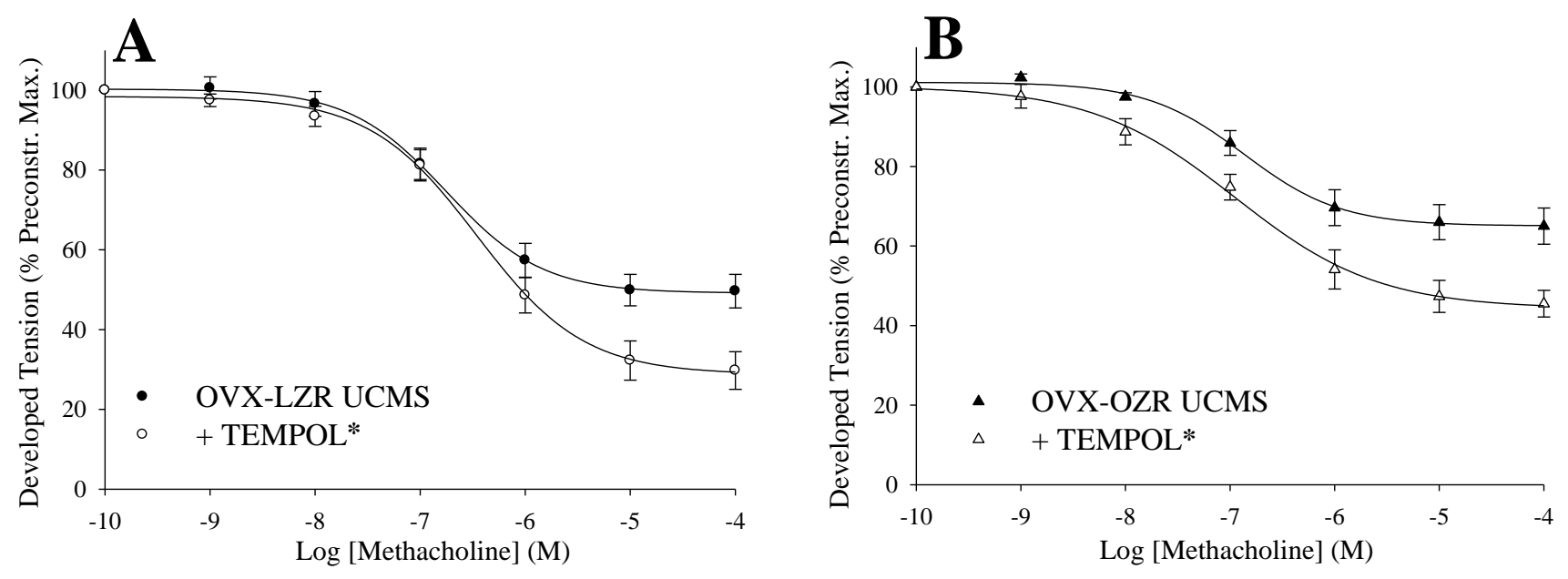

Figure 10

Stanley et al. 


\section{CHAPTER 2: CONCEPTUAL FRAMEWORK}

The conclusions from the study presented in this chapter addressed each of the three main questions that arose from the work presented in chapter one. These findings can be summarized as follows:

-Imposition of UCMS on both male and female LZR and OZR resulted in the development of depressive behaviors, as well as impairment of endothelial function in aortic rings of all animals. As expected with UCMS, female LZR developed more severe depressive behaviors, yet females exhibited less severe impairment of endothelium-dependent dilation to methacholine compared to males. UCMS resulted in hyperglycemia in male and female LZR, along with increased levels of the corticosterone and inflammatory biomarkers TNF- $\alpha$, IL-1 $\beta$, and IL-6.

- Pre- treatment with L-NAME in aortic rings had minimal effects on vascular reactivity in UCMS male LZR, while the blunted dilator responses were further impaired as a result of COX inhibition with indomethacin, suggesting that a dependence on dilator influence of arachidonic acid metabolites (likely $\mathrm{PGI}_{2}$ ) helps to maintain vascular reactivity in the compromised state. In contrast, L-NAME blocked dilation in UCMS female LZR, while indomethacin had a less striking effect. These findings largely corroborate with those of chapter 1 , and confirm that the behavioral and vascular effects of UCMS in mice extend across species to that of a rat model.

- Imposition of UCMS on male and female OZR resulted in the worst behavioral outcomes, as well as the most severe impairments in vascular function. Importantly, the vascular protection to UCMS-induced vasculopathy observed in lean females was no longer exhibited in female OZR, as blunted vasodilator response to agonist challenge was not significantly different from that of male OZR (nor in behavior outcome). Plasma glucose, corticosterone, and inflammatory biomarkers were all significantly elevated in UCMS male and female OZR compared to UCMS male and female LZR. Overall, the co-morbid presence of pre-existing metabolic syndrome resulted in amplification of the deleterious effects of UCMS on the vasculature, and eliminated the sex-specific vascular protection seen in UCMS female LZR. Interestingly, even in the absence of exogenous stress, male and female OZR (control) developed depressive like symptoms, suggesting the presence of a bidirectional relationship between CVD and depression observed in clinical studies showing that adverse CV events are risk factors for depression. Alternatively, this may be related to the genetic make-up of the OZR, which possess a mutation in the 
leptin receptor (LepRb), resulting in chronically elevated leptin and impaired leptin signaling in the brain, which has been linked to both central and peripheral processes, as well as depressive behaviors.

- Finally, UCMS-induced vascular dysfunction was increased while behavioral outcomes were attenuated in OVX animals, both lean and obese. Generally, the effects of OVX had a greater impact on vascular function in LZR (vs OZR), resulting in similar levels of impaired behavioral and vascular responses to that of UCMS male LZR. OVX LZR and OZR demonstrated elevated plasma glucose and inflammatory markers compared to control female LZR and OZR, respectively. These results suggest that loss of female sex hormones contribute to a pro-inflammatory and pro-oxidative environment in the vasculature.

In sum, the results of this study strongly support the role of inflammation and reduced NO bioavailability in the development of depressive behaviors and vascular impairments in both male and female, and lean and obese, animals. The loss of female estrogen resulted in elevated levels of inflammation and worsened vascular responses, suggesting that normal hormone levels in female animals positively regulate NO bioavailability and reduce the deleterious effects on vascular inflammation. As outlined in the introduction to this dissertation, the sex hormone estrogen has vasculoprotective effects via promotion of vascular NO production by endothelium and enhancement of anti-oxidant defenses.

The spontaneous development of depressive symptoms in OZR demonstrated by these results is supported by previous behavioral studies using this animal model, and this brings up an interesting avenue for further investigation. As increasing evidence supports the concept of clinical depression as a disease of chronic sub-acute inflammation, with clear parallels in terms of biomarker expression that are comparable to those for elevated CVD risk, would regulation of inflammation and/or oxidative stress, through either sustained physical exercise or pharmacological interventions, lead to improved vascular and behavioral outcomes? Is there an intrinsic property within evolution of the metabolic syndrome that can cause development of depressive symptoms in rodents? Treatments that target systemic pathologies of the metabolic syndrome may significantly improve both cardiovascular and behavioral function, resulting in the most beneficial effects on health outcomes. The third chapter of this dissertation will then aim to determine if chronic treatment of CVD risk factors will improve both vascular function and behavioral responses. To investigate this, a cohort of male OZR was subjected to 9 to 10- 
weeks of treadmill exercise or chronic treatment with different pharmacological agents commonly used to control risk factors of metabolic syndrome 
Chapter 3

INTERVENTIONS BLUNTING SYSTEMIC INFLAMMATION AND OXIDANT STRESS ARE REQUIRED TO IMPROVE VASCULAR FUNCTION AND DEPRESSIVE SYMPTOMS IN METABOLIC SYNDROME

Shyla C. Stanley ${ }^{1,7}$, Alexandre C. d'Audiffret ${ }^{2,6,7}$, Khumara Huseynova ${ }^{2,6,7}$, Lawrence E. Tabone ${ }^{3,6,7}$, Kristin A. Grogg ${ }^{6,7}$, Stephanie J. Frisbee ${ }^{4,6,7}$, Carl D. Shrader ${ }^{5,6,7}$, and Jefferson C. Frisbee ${ }^{1,6,7}$

Department of Physiology and Pharmacology ${ }^{1}$, Division of Vascular and Endovascular Surgery ${ }^{2}$, Division of Bariatric Surgery ${ }^{3}$, Department of Health Policy, Management and Leadership ${ }^{4}$, Department of Family Medicine ${ }^{5}$, Clinical and Translational Sciences Institute ${ }^{6}$, Center for Cardiovascular and Respiratory Sciences ${ }^{7}$; West Virginia University Health Sciences Center, Morgantown, WV

Running Head: Metabolic syndrome, depressive symptoms and vascular function

Send Correspondence to:

Jefferson C. Frisbee, Ph.D.,

Center for Cardiovascular and Respiratory Sciences

Department of Physiology and Pharmacology

Robert C. Byrd Health Sciences Center

PO Box 9105

West Virginia University School of Medicine

Morgantown, WV 26505

Phone: (304) 293-6527

Fax: (304) 293-5513

Email: jefrisbee@hsc.wvu.edu 


\section{ABSTRACT}

While the increased prevalence and severity of chronic depressive symptoms and elevated cardiovascular disease risk represent two of the most challenging public health issues in developed economies, the growing realization of their combined presentation presents a special challenge for not only cardiovascular health outcomes, but also for effective treatment/interventions. The obese Zucker rat (OZR), a model of the metabolic syndrome with significant elevations in cardiovascular disease (CVD) risk spontaneously develops significant depressive symptoms in parallel with the progression of the metabolic syndrome; thus representing a particularly compelling model for study. To interrogate the relationships between CVD risk factors and depressive symptoms, we chronically treated OZR with traditional interventions targeted against CVD risk to determine the impacts on not only vascular function but also on behavioral outcomes and depressive symptom severity. While most of the employed interventions (chronic exercise, anti-hypertensive, anti-dyslipidemia, anti-diabetic) were differentially effective at improving vascular function, only those interventions that also resulted in a significant improvement to both oxidant stress and inflammation and their associated sequelae were also effective at blunting the severity of depressive symptoms. This initial study provides for a very compelling framework from which to further interrogate the links between CVD risk and cognitive/behavioral status, and begins to provide insight into the mechanistic links between the two and potentially effective avenues for intervention. 


\section{INTRODUCTION}

It is well known that the growing incidence and prevalence of the metabolic syndrome presents a consistent threat to aggregate public health cardiovascular outcomes across many societies $(1,11,24,28,33)$. This syndrome is broadly defined as the combined presentation of obesity, impaired glycemic control, atherogenic dyslipidemia, and hypertension, with the additional contributing conditions of a pro-oxidant, -thrombotic, and inflammatory phenotype $(11,24)$. This clinical condition afflicts a growing number of patients worldwide, demonstrating substantial reduction in both patient quality of life $(1,28)$ and life expectancy $(24,33)$. Given both the individual detriment to patient health, and the substantial societal economic burden in terms of direct and indirect economic costs, $(20,35,37)$, further detailed investigations into this multi-pathology state are warranted.

The obese Zucker rat (OZR; $f a / f a)$ is an excellent model for the development of the metabolic syndrome and the associated negative vascular outcomes in humans. Due to a genetic mutation in the leptin receptor that causes severe leptin resistance, OZR manifest chronic hyperphagia that ensures rapid development of the systemic pathologies (previously mentioned) to the degree of severity characterized in human subjects. In addition to metabolic syndrome, OZR suffer from a progressive vasculopathy that ultimately progresses into overt peripheral vascular disease $(26,40)$, albeit one in the absence of significant atherosclerotic lesions. However, it has been recently determined that OZR progressively develop compromised behavioral patterns (without use of additional intervention treatments) indicative of chronic stress and depressive symptoms (2), as well as significantly elevated circulating cortisol and corticosterone levels $(6,10,29)$.

This is particularly intriguing as the presence of chronic stress and depressive symptoms have been identified as a powerful, independent risk factor for negative cardiovascular and cerebrovascular $(31,38,39)$ outcomes. The significant reduction in patient quality of life, profound economic costs, and extreme social burden associated with depressive disorders $(7,21,22,27,32,41)$, coupled with the adverse societal effects of metabolic syndrome, present a global health problem that has emerged as one of the most challenging public health issues to date as the combined presence of these diseases continue to rise. While the use of selective serotonin/norepinephrine reuptake inhibitors (SSRI/SNRI), tricyclic antidepressants, and other pharmacological agents against depressive symptoms has been extensively investigated $(9,25)$, specifically with regards to their 
potential impact on cardiovascular function $(5,12,25)$, the spontaneous development of significant depressive symptoms in OZR suggests a different approach may be warranted - that of treating the constituent pathologies of the metabolic syndrome and determining the impact on depressive symptomology.

The overarching purpose of the present study was to determine the efficacy of chronic interventions (both pharmacological and physiological) in preventing the development of both behavioral and vascular impairments that progressively occur with age in OZR. Experimental interventions targeting pathological components of the metabolic syndrome were implemented at an early age, preceding the development of CVD and depressive symptoms in OZR, to determine the most beneficial venues for improving ex vivo vascular function and blunting/reversing depressive symptom severity. With the appropriate and judicious use of chronic interventions, and analytical approaches, the secondary purpose of the present study was to provide justified identification of the central mechanistic links which exist between metabolic syndrome, vascular/perfusion dysfunction, and depressive symptomology. The present study was designed to test the integrated hypothesis that chronic targeted

treatment against the systemic progressive pathologies of the metabolic syndrome results in significant improvements to both cardiovascular and behavioral function and provide for the maximum potential improvements to long term health outcomes.

\section{MATERIALS AND METHODS}

Animals: Male lean (LZR) and obese Zucker rats (OZR) were delivered to the West Virginia University Health Sciences Center at 6-7 weeks of age, and after one week of acclimation to the local environment, we placed into a specific protocol for the subsequent 9-10 weeks. Unless otherwise stated (below), all animals were fed standard chow and tap water ad libitum for all experiments. All rats were housed in the animal care facility at the West Virginia University Health Sciences Center, and all protocols received prior IACUC approval. At 7-8 weeks of age, LZR and OZR ( $\mathrm{n}=12$ for each group) were placed into one of the following groups for the subsequent 9-10 weeks:

1. Time control (normal food and water ad libitum)

2. Chronic treadmill exercise ( $20 \mathrm{~m} / \mathrm{min}, 5 \%$ incline, 60 minutes/day, 6 days/week) 
3. Anti-hypertensive treatment with captopril (angiotensin converting enzyme inhibitor; $60 \mathrm{mg} \bullet \mathrm{kg}^{-1} \bullet \mathrm{day}^{-1}$; drinking water; 17)

4. Anti-hypertensive treatment with hydralazine (systemic vasodilator; $50{\mathrm{mg} \bullet \mathrm{kg}^{-1} \bullet \text { day }}^{-1}$; drinking water; 17)

5. Anti-diabetic treatment with metformin (hepatic gluconeogenesis inhibitor; $300 \mathrm{mg} \bullet \mathrm{kg}^{-1} \bullet \mathrm{day}^{-1}$; drinking water; 17)

6. Anti-diabetic treatment with rosiglitazone (insulin sensitizing agent; $10 \mathrm{mg} \bullet \mathrm{kg}^{-1} \bullet$ day ${ }^{-1}$; mixed with food; 17)

7. Anti-dyslipidemia treatment with atorvastatin (HMG Co-A reductase inhibitor; $25 \mathrm{mg} \mathrm{kg}^{-1} \bullet \mathrm{day}^{-1}$; mixed with food; 17)

8. Anti-dyslipidemia treatment with gemfibrozil (peroxisome proliferator-activated receptor alpha (PPAR $\alpha$ ) activator; $100 \mathrm{mg} \bullet \mathrm{kg}^{-1} \bullet \mathrm{day}^{-1}$; mixed with food; 17)

9. Anti-oxidant treatment with TEMPOL $\left(10^{-3} \mathrm{M}\right.$; drinking water; 17)

Following completion of the 9-10 week treatment period, a behavioral assessment was conducted on each animal that included a determination of coat status (36).

Coat Status/Score: This evaluation addresses chronic grooming behavior. A research assistant completed a visual inspection of the animal prior to usage and evaluated the coat status of the animals. A total cumulative score was computed by giving an individual score of 0 (clean) or 1 (dirty) to eight body parts (head, neck, dorsal coat, ventral coat, tail, forelimb, hind-limb, and genital region).

At the time of final usage, rats were anesthetized with injections of sodium pentobarbital $\left(50 \mathrm{mg} \mathrm{kg}^{-1}\right.$ i.p.), and all rats received tracheal intubation to facilitate maintenance of a patent airway. In all rats a carotid artery and an external jugular vein were cannulated for determination of arterial pressure and for intravenous infusion of additional substances as necessary (e.g., anesthetic, heparin, etc.). In addition, an aliquot of mixed venous blood was drawn from the jugular vein cannula for a full profiling of metabolic, endocrine and inflammatory biomarkers (see below), and each animal received a bolus injection of heparin to temporarily reduce the occurrence of blood coagulation without jeopardizing vascular reactivity $\left(100 \mathrm{IU} \bullet \mathrm{kg}^{-1}\right)$.

Skeletal Muscle Arteriolar Reactivity: Following the initial surgery, an incision was made into the medial upper hind limb and the extra-parenchymal resistance artery/first order arteriole supplying the gracilis muscle was 
identified. This vessel was cleared of all surrounding tissue along its length and into the gracilis muscle. Subsequently, the section of the vessel that was fully within the gracilis muscle was removed and doubly cannulated on glass micropipettes using a previously well-established procedure (3). At this point, hind limb conduit arteries were removed from the rat (e.g., femoral, popliteal, saphenous), cleaned, and placed in cold PSS for subsequent determination of specific signaling metabolites relevant for vascular reactivity (see below). The reactivity of isolated arteries was assessed in response to increasing concentrations of acetylcholine $\left(10^{-10} \mathrm{M}-10^{-6}\right.$ M).

Determination of Signaling Molecule Bioavailability: All harvested conduit arteries from each rat were lightly sectioned into segments of $\sim 1 \mathrm{~mm}$ in length and placed in a chamber for study, superfused with warmed $\left(37^{\circ} \mathrm{C}\right)$ PSS equilibrated with $95 \% \quad \mathrm{O}_{2}$ and $5 \% \quad \mathrm{CO}_{2}$. Vascular nitric oxide (NO) production was assessed using amperometric sensors (World Precision Instruments, Sarasota, FL). A NO sensor (ISO-NOPF 100) was inserted into the chamber and a baseline level of current was obtained. Subsequently, increasing concentrations of methacholine $\left(10^{-10}-10^{-6} \mathrm{M}\right)$ were added to the bath and the changes in current were determined. Subsequently, vascular production of 6-keto-prostaglandin $\mathrm{F}_{1 \alpha}\left(6-\mathrm{keto}_{\mathrm{PGF}}\right.$; the stable breakdown product of $\left.\mathrm{PGI}_{2}\right)$, and 11dehydro-thromboxane $\mathrm{B}_{2}$ (11-dehydro- $\mathrm{TxB}_{2}$; the stable plasma breakdown product of $\mathrm{Tx}_{2}$ ) in response to challenge with arachidonic acid $\left(10^{-5} \mathrm{M}\right)$ was measured. Pooled, sectioned arteries were left untreated for 30 minutes under control conditions (after which the superfusate was removed and frozen in liquid $\mathrm{N}_{2}$ ) and were allowed to incubate with the introduced arachidonic acid for an additional 30 minutes (followed by superfusate removal and storage). Vessel weights were determined after blotting on gauze to remove excess liquid, and metabolite release by the vessels was determined using commercially available EIA kits for 6-keto-PGF ${ }_{1 \alpha}$ and 11dehydro- $\mathrm{TxB}_{2}$ (Cayman).

Data and Statistical Analyses: To simplify analyses, individual biomarkers of key parameters were combined into groups to provide aggregate estimators of the severity of the degree of dysfunction. While coat score and cortisol levels were not manipulated, integrated vascular reactivity was estimated as the upper bound of the acetylcholine concentration-response relationship (see below). The levels of glycemic control, referred to HOMA, was estimated as the product of plasma insulin concentration multiplied by blood glucose concentration, 
where higher values clearly represent poorer glycemic control. The severity of the oxidant stress and inflammatory state, referred to as Oxidant Load, was defined as the product of plasma nitrotyrosine concentration multiplied by plasma TNF- $\alpha$ concentration, with that product divided by the slope of the nitric oxide bioavailability curve, where higher values represents pro-oxidant, pro-inflammatory states with low NO bioavailability. The balance of arachidonic acid metabolism in the vasculature was estimated as the quotient of $\mathrm{PGI}_{2}$ and $\mathrm{TxA}_{2}$ production, and is referred to as AA Met Balance, where higher values represent optimal health and lower CVD risk.

All data are presented as mean \pm SEM. Statistically significant differences in measured physiological parameters, calculated physiological parameters (e.g., slope coefficients, upper or lower bounds), measurements of plasma biomarkers, were determined using analysis of variance (ANOVA). In all cases, Student-NewmanKeuls post hoc test was used when appropriate and $\mathrm{p}<0.05$ was taken to reflect statistical significance. The mechanical responses of ex vivo microvessels following agonist challenge were fit with the three-parameter logistic equation:

$$
y=\min +\left[\frac{\max -\min }{1+10^{\log E D_{50}-x}}\right]
$$

where ${ }^{y}$ represents the change in arteriolar diameter, "min" and "max" represent the lower and upper bounds, respectively, of the change in diameter or tension with increasing agonist concentration, $x$ is the logarithm of the agonist concentration and $\log \mathrm{ED}_{50}$ represents the logarithm of the agonist concentration $\left({ }^{x}\right)$ at which the response $\left({ }^{y}\right)$ is halfway between the lower and upper bounds.

Vascular NO bioavailability measurements were fit with a linear regression equation $\left(y=\alpha_{0}+\beta_{1} x\right)$; where ${ }^{y}$ represents the NO concentration, $\propto_{0}$ represents an intercept term, $\beta_{1}$ represents the slope of the relationship, and $x$ represents the log molar concentration of methacholine. 


\section{RESULTS}

Table 1 summarizes the baseline characteristics of animals utilized in the present study. While OZR were consistently heavier than LZR and demonstrated all of the phenotypes of the metabolic syndrome, the employed interventions were effective at reducing the severity of their respective CVD risks, with exercise being able to broadly reduce CVD risk in parallel with the reduced severity of obesity.

Figure 1 presents the univariate relationship between individual animal cortisol and coat score for control LZR and OZR (Panel A) and for all of the animals (Panel B) within the present study. As is clearly evident from these data, there was a strong positive relationship between the levels of plasma cortisol and the degradation in the coat status for the individual animals. This provides a strong level of support for the overriding conceptual model for the present experiment is that coat score and plasma cortisol levels are general indicators of behavioral status (chronic stress and depressive symptoms) in LZR and OZR.

The initial analyses of the data focused on a univariate approach correlating our predictive markers to outcomes. Figure 2 (for coat score) and Figure 3 (for plasma cortisol) summarizes these basic analyses, and clearly demonstrates a robust univariate relationship between our predictive markers of the upper bound of the acetylcholine concentration-response relationship (Panel A), our measure of insulin resistance (Panel B), our integrated measurement of oxidant/inflammation stress (Panel C) and the measurement of altered arachidonic acid metabolism (Panel D) for control LZR and OZR. However, the extent to which these parameters are correlated to the coat score and plasma cortisol outcomes is less robust as data from OZR following the imposed interventions of the present study for the acetylcholine upper bound (Panel E), insulin resistance (Panel F), oxidant/inflammation stress (Panel G) and arachidonic acid metabolism (Panel H).

Given the increasing weakness of the relationships between the predictive parameter and the behavioral outcomes, it was necessary to identify the relationships between coat score, plasma cortisol and our predictive parameters. Figure 4 presents the relationship between coat score, cortisol and the upper bound of the acetylcholine-dose response relationship in control LZR and OZR. This relationship (which is qualitatively very similar to the other three predictors; data not shown) clearly demonstrates the negative correlations between upper bound and either cortisol levels or coat score, while also demonstrating the positive relationship between 
coat score and cortisol levels (Panel A). The individual animal relationships grouping coat score, plasma cortisol and acetylcholine upper bound are summarized in Panel B, which demonstrates the effectiveness of chronic treatments with TEMPOL or atorvastatin or chronic exercise in terms of improving both vascular and behavioral outcomes, while chronic gemfibrozil, anti-hypertensive, or anti-diabetic treatments were less effective at improving aggregate outcomes. However, for the interventions that resulted in a significant improvement to coat score and cortisol (chronic exercise, atorvastatin and TEMPOL treatment), this was associated with a significant improvement to the upper bound of the acetylcholine concentration-response relationship (Panel C), while those interventions that did not result in an improvement to the behavioral markers of coat score and cortisol levels (gemfibrozil, metformin, hydralazine, rosiglitazone), were also not associated with an improvement to vascular function (from the upper bound relationship; Panel D).

Figure 5 presents the data describing the ability of the interventions from the present study to restore normal function (that determined in LZR control) from the maximum level of dysfunction (OZR control) for the parameter in question. For both coat score (Panel A) and cortisol (Panel B), chronic exercise, atorvastatin and TEMPOL treatment resulted in a consistent restoration of greater than $30-40 \%$ of the maximum possible (i.e., full) recovery in terms of these two parameters describing behavioral outcomes. In contrast, treatment with gemfibrozil, metformin, captopril, hydralazine, or rosiglitazone had a much less significant and consistent effect on either coat score or plasma cortisol levels. Building on this, the impact of the chronic interventions to improve vascular reactivity (Panel C), glycemic control (Panel D), oxidant stress and inflammation (Panel E) and to restore normal arachidonic acid metabolism (Panel F), all demonstrated a similar trend, with exercise and atorvastatin treatments being most effective at improving these parameters back to the levels determined in LZR controls. With the single exception of glycemic control, chronic TEMPOL treatment was also most consistently effective at restoring the four parameters to normal levels of function. 


\section{DISCUSSION}

One of the more intriguing observations from epidemiological and public health research is the remarkably tight relationship that exists between the prevalence and severity of depressive symptoms within the population and the concurrent development of elevated cardiovascular disease risk. This opens up wide series of questions and issues to be addressed in terms of the parallel development of these systemic conditions, their health outcomes, issues of disparity, and policy adaptations to address this, but these are outside of the scope of the present study.

While there exist a wide array of studies demonstrating a variable level of efficacy of classic pharmacological interventions for the treatment of depressive symptoms $(9,25)$, many of these have a wide array of undesirable, and at times long-lasting, side effects that can limit patient adherence $(5,9)$ or result in a "trade off" of one compromised facet of quality of life for others.

In preliminary studies, we have recently determined that the OZR begins to develop many of the hallmarks of increased depressive symptom severity in parallel to the development of the constituent pathologies of the metabolic syndrome (2). These observations include a steadily decreasing level of physical activity/increasingly sedentary behavior, an increased plasma cortisol level, and a steady, progressive failure to groom that precedes the development of severe obesity (which would physically preclude grooming). As has also been repeatedly demonstrated by multiple laboratories, the OZR also develops significant impairments to normal vascular function, including alterations to dilator $(13,19,23,43)$ and constrictor $(4,14,16,30)$ reactivity, vessel structure (18), vascular network structure (15) and perfusion responses (16, 34, 42), in the skeletal muscle circulation. Clearly, with the myriad alterations occurring in parallel in the OZR, there is the potential for these to create integrated systems where one impairment to vascular function, can predispose the animal to impaired cerebral function (or vice versa), with the aggregate output being a progressive development of the symptomology of both peripheral vascular disease and depressive behaviors. The purpose of the present study was to determine if chronic interventions targeted at cardiovascular disease risk reduction would not only improve vascular outcomes, but if this would also improve behavioral responses through a reduction in markers of depressive symptom severity. 
The initial responses of the present study demonstrate that the five employed predictors of the general behavioral response (coat score or cortisol) are all strong, univariate correlates of the behavioral outcomes in control (untreated) LZR and OZR throughout the age ranges from 7-8 weeks to $\sim 17$ weeks (Figures 2 and 3). However, when additional data are added following the interventions, the correlations are weakened considerably, suggesting that some of the interventions result in a condition wherein the ability of the outcome "vascular" parameters to predict behavioral responses becomes less robust. This strongly suggests that the conditions that are created, although potentially improving vascular outcomes are not sufficient to impact behavioral ones, and thus are less effective treatments overall. However, this analysis is insufficient from which to draw further insight.

Building on this, we determined the "normal" relationship between vascular function, behavioral outcomes (i.e., coat score) and plasma cortisol (as the parameter that can link the two). These data, summarized in Figure 4, provide for a robust understanding of this relationship, where poor vascular function (low upper bounds for the acetylcholine concentration-response relationship) was associated with high levels of plasma cortisol and high coat scores. Using the present data, it is relatively clear to see that few interventions (i.e., chronic exercise, TEMPOL and atorvastatin) were most effective at improving coat score in OZR, while the other interventions, while still effective at improving specific outcomes, produced a less robust improvement to the behavioral measures. When compared to the normal relationships between vascular function, cortisol and coat score between LZR and OZR, it is clear that the three successful interventions were more effective at shifting the aggregate relationship to improved vascular function, lower cortisol and improved coat score, while those interventions that were less effective may have improved vascular function per se, but were less effective at impacting cortisol or coat score.

This determination led to the obvious question of what distinguishes one intervention from the others in terms of improving both behavioral and vascular outcomes. To address this, we utilized our six parameters of outcomes (coat score, cortisol, ACh upper bound, HOMA, oxidant load and AA metabolism balance) and determined the ability of the individual interventions to restore normal function (i.e., that determined in LZR) 
from the maximum degree of impaired function (i.e., that determined in OZR), with full restoration defined as $100 \%$.

The results of these analyses clearly demonstrated the divergence in the effectiveness of treatment interventions. Chronic exercise and chronic treatment with atorvastatin were consistently able to cause significant improvements to all of the key parameters utilized for the present study. Similarly, chronic treatment with TEMPOL was also extremely effective at improving 5 of the 6 outcome parameters of the present study. In contrast none of the other imposed treatments were able to cause significant improvements to more than 3 or 4 (captopril only) of the key parameters utilized.

These data raise the clear question of what are the mechanistic underpinnings of the most effective interventions such that they were able to improve both vascular and behavioral function significantly. While a detailed determination of these mechanisms and how they interrelate will likely be quite complex and is well beyond the scope of a single manuscript, clear inferences can be derived based on what was and what was not effective. Clearly, in order for the intervention to be effective, the primary outcome must be a significant attenuation of both chronic inflammation and chronic elevations to oxidant stress. While these are both wellestablished outcomes of chronic exercise (15), chronic treatment with TEMPOL (13) and as the pleiotropic effects of chronic treatment with atorvastatin (18), their effect on improving outcomes appears to go beyond simply improving vascular function (as other interventions that also improve vascular function are less effective at correcting behavioral outcomes).

Given an increasing emphasis and recognition of both cardiovascular disease risk factors $(8,11,28)$ and clinical depression $(9,25)$ as diseases strongly associated with chronic elevations in oxidant stress and sub-acute inflammation, it is perhaps not surprising that these three interventions (exercise, TEMPOL and atorvastatin) are most able to positively impact both vascular function and behavioral outcomes. However, while these studies are very provocative, they highlight the need to identify whether these relationships are also present in the cerebral circulation, which may have a much more direct impact on the behavioral responses, and if there are specific mechanistic links at a higher resolution that can be exploited with future interventional efforts. Most importantly, 
these results highlight the potential utility of a novel use of established therapeutics for CVD risk that may provide significant relief from the devastating impact of chronic depressive symptoms.

\section{ACKNOWLEDGEMENTS}

The authors would like to thank Ms. Milinda James for her expert technical assistance. Additionally, we also acknowledge the support provided through Center for Cardiovascular and Respiratory Sciences and the Clinical and Translational Sciences Institute at the West Virginia University Health Sciences Center.

\section{SOURCES OF RESEARCH FUNDING}

This study was supported by the American Heart Association (IRG 14330015, PRE 16850005, EIA 0740129N), and the National Institutes of Health (U54GM104942; RR 2865AR; P20 RR 016477). Research reported in this publication was supported by the National Institute of General Medical Sciences of the National Institutes of Health under Award Number U54GM104942. The content is solely the responsibility of the authors and does not necessarily represent the official views of the National Institutes of Health. 


\section{REFERENCES}

1. Amihăesei IC, Chelaru L. Metabolic syndrome a widespread threatening condition; risk factors, diagnostic criteria, therapeutic options, prevention and controversies: an overview. Rev Med Chir Soc Med Nat Iasi. 2014; 118(4):896-900.

2. Brooks SD, Stanley SC, Butcher JT, d'Audiffret A, Skaff PR, Chantler PD, Frisbee JC. Severity of behavioral impairments and vascular dysfunction with chronic stress/depressive symptoms is increased by metabolic syndrome. (Experimental Biology, 2014 San Diego, CA)

3. Butcher JT, Goodwill AG, Frisbee JC. The ex vivo isolated skeletal microvessel preparation for investigation of vascular reactivity. J Vis Exp. 2012; 28;(62): 3674.

4. Butcher JT, Goodwill AG, Stanley SC, Frisbee JC. Differential impact of dilator stimuli on increased myogenic activation of cerebral and skeletal muscle resistance arterioles in obese zucker rats. Microcirculation. 2013; 20(7):579-89.

5. Chittaranjan A, Chethan KB, Sandarsh S. Cardiovascular mechanisms of SSRI drugs and their benefits and risks in ischemic heart disease and heart failure. Int Clin Psychopharmacol. 2013;28(3):145-55.

6. Clark RG, Thomas GB, Mortensen DL, Won WB, Ma YH, Tomlinson EE, Fairhall KM, Robinson IC. Growth hormone secretagogues stimulate the hypothalamic-pituitary-adrenal axis and are diabetogenic in the Zucker diabetic fatty rat. Endocrinology. 1997; 138(10):4316-23.

7. Cuijpers P, Vogelzangs N, Twisk J, Kleiboer A, Li J, Penninx BW. Differential mortality rates in major and subthreshold depression: meta-analysis of studies that measured both. Br J Psychiatry. 2013; 202(1):22-7.

8. de Carvalho Vidigal F, Bressan J, Babio N, Salas-Salvadó J. Prevalence of metabolic syndrome in Brazilian adults: a systematic review. BMC Public Health. 2013; 13:1198.

9. Dhillon S. Duloxetine: a review of its use in the management of major depressive disorder in older adults. Drugs Aging. 2013; 30(1):59-79. 
10. Duclos M, Timofeeva E, Michel C, Richard D. Corticosterone-dependent metabolic and neuroendocrine abnormalities in obese Zucker rats in relation to feeding. Am J Physiol Endocrinol Metab. 2005; 288(1):E254-66.

11. Falkner B, Cossrow ND. Prevalence of metabolic syndrome and obesity-associated hypertension in the racial ethnic minorities of the United States. Curr Hypertens Rep. 2014; 16(7):449.

12. Fiedorowicz JG. Depression and cardiovascular disease: an update on how course of illness may influence risk. Curr Psychiatry Rep. 2014; 16(10):492.

13. Frisbee JC, Hollander JM, Brock RW, Yu HG, Boegehold MA. Integration of skeletal muscle resistance arteriolar reactivity for perfusion responses in the metabolic syndrome. Am J Physiol Regul Integr Comp Physiol. 2009; 296(6):R1771-82.

14. Frisbee JC, Maier KG, Stepp DW. Oxidant stress-induced increase in myogenic activation of skeletal muscle resistance arteries in obese Zucker rats. Am J Physiol Heart Circ Physiol. 2002; 283(6):H2160-8.

15. Frisbee JC, Samora JB, Peterson J, Bryner R. Exercise training blunts microvascular rarefaction in the metabolic syndrome. Am J Physiol Heart Circ Physiol. 2006; 291(5):H2483-92.

16. Frisbee JC. Enhanced arteriolar alpha-adrenergic constriction impairs dilator responses and skeletal muscle perfusion in obese Zucker rats. J Appl Physiol (1985). 2004; 97(2):764-72.

17. Goodman and Gilman's The Pharmacological Basis of Therapeutics, $11^{\text {th }}$ Edition. Eds: LL Brunton, JS LAzo, KL Parker. McGraw-Hill Professional. 2005

18. Goodwill AG, Frisbee SJ, Stapleton PA, James ME, Frisbee JC. Impact of chronic anticholesterol therapy on development of microvascular rarefaction in the metabolic syndrome. Microcirculation. 2009; 16(8):667-84.

19. Goodwill AG, James ME, Frisbee JC. Increased vascular thromboxane generation impairs dilation of skeletal muscle arterioles of obese Zucker rats with reduced oxygen tension. Am J Physiol Heart Circ Physiol. 2008; 295(4):H1522-8.

20. Gulbins H, Vogel B, Reichenspurner H. Gender effects on health care costs in cardiovascular medicine-a black box? Thorac Cardiovasc Surg. 2013; 61(1):74-8. 
21. Halaris A. Inflammation, heart disease, and depression. Curr Psychiatry Rep. 2013;15(10):400.

22. Hare DL, Toukhsati SR, Johansson P, Jaarsma T. Depression and cardiovascular disease: a clinical review. Eur Heart J. 2014; 35(21):1365-72.

23. Hodnett BL, Dearman JA, Carter CB, Hester RL. Attenuated $\mathrm{PGI}_{2}$ synthesis in obese Zucker rats. Am J Physiol Regul Integr Comp Physiol. 2009; 296(3):R715-21.

24. Hutchinson RN, Shin S. Systematic review of health disparities for cardiovascular diseases and associated factors among American Indian and Alaska Native populations. PLoS One. 2014; 9(1):e80973.

25. Katsi VK, Marketou M, Vamvakou G, Makris T, Tousoulis D, Stefanadis CI, Vardas P, Kallikazaros IE. Novel antidepressant drugs, arterial hypertension and cardiovascular disease. Recent Pat Cardiovasc Drug Discov. 2013; 8(3):178-85.

26. Katsuda Y, Ohta T, Miyajima K, Kemmochi Y, Sasase T, Tong B, Shinohara M, Yamada T. Diabetic complications in obese type 2 diabetic rat models. Exp Anim. 2014; 63(2):121-32.

27. Kessler RC, Bromet EJ. The epidemiology of depression across cultures. Annu Rev Public Health. 2013; 34:119-38.

28. l'Allemand-Jander D. Clinical diagnosis of metabolic and cardiovascular risks in overweight children: early development of chronic diseases in the obese child. Int J Obes (Lond). 2010; 34 Suppl 2:S32-6.

29. Livingstone DE, Jones GC, Smith K, Jamieson PM, Andrew R, Kenyon CJ, Walker BR. Understanding the role of glucocorticoids in obesity: tissue-specific alterations of corticosterone metabolism in obese Zucker rats. Endocrinology. 2000; 141(2):560-3.

30. Naik JS, Xiang L, Hester RL. Enhanced role for RhoA-associated kinase in adrenergic-mediated vasoconstriction in gracilis arteries from obese Zucker rats. Am J Physiol Regul Integr Comp Physiol. 2006; 290(1):R154-61. 
31. Najjar S, Pearlman DM, Devinsky O, Najjar A, Zagzag D. Neurovascular unit dysfunction with blood-brain barrier hyperpermeability contributes to major depressive disorder: a review of clinical and experimental evidence. J Neuroinflammation. 2010; 1:10:142.

32. Park M, Katon WJ, Wolf FM. Depression and risk of mortality in individuals with diabetes: a meta-analysis and systematic review. Gen Hosp Psychiatry. 2013; 35(3):217-25.

33. Saito I. Epidemiological evidence of type 2 diabetes mellitus, metabolic syndrome, and cardiovascular disease in Japan. Circ J. 2012; 76(5):1066-73.

34. Sebai M, Lu S, Xiang L, Hester RL. Improved functional vasodilation in obese Zucker rats following exercise training. Am J Physiol Heart Circ Physiol. 2011; 301(3):H1090-6.

35. Shamseddeen H, Getty JZ, Hamdallah IN, Ali MR. Epidemiology and economic impact of obesity and type 2 diabetes. Surg Clin North Am. 2011; 91(6):1163-72.

36. Stanley SC, Brooks SD, Butcher JT, d'Audiffret AC, Frisbee SJ, Frisbee JC. Protective effect of sex on chronic stress- and depressive behavior-induced vascular dysfunction in BALB/cJ mice. J Appl Physiol (1985). 2014; 1;117(9):959-70.

37. Trasande L, Elbel B. The economic burden placed on healthcare systems by childhood obesity. Expert Rev Pharmacoecon Outcomes Res. 2012; 12(1):39-45.

38. Tully PJ, Baumeister H. Collaborative care for the treatment of comorbid depression and coronary heart disease: a systematic review and meta-analysis protocol. Syst Rev. 2014; 28(3):127.

39. Tully PJ, Cosh SM, Baumeister H. The anxious heart in whose mind? A systematic review and metaregression of factors associated with anxiety disorder diagnosis, treatment and morbidity risk in coronary heart disease. J Psychosom Res. 2014; 77(6):439-48.

40. Wang B, Chandrasekera PC, Pippin JJ. Leptin- and leptin receptor-deficient rodent models: relevance for human type 2 diabetes. Curr Diabetes Rev. 2014;10(2):131-45.

41. Whiteford HA, Degenhardt L, Rehm J, Baxter AJ, Ferrari AJ, Erskine HE, Charlson FJ, Norman RE, Flaxman AD, Johns N, Burstein R, Murray CJ, Vos T. Global burden of disease attributable to mental and 
substance use disorders: findings from the Global Burden of Disease Study 2010. Lancet. 2013; 9; 382(9904):1575-86.

42. Xiang L, Naik J, Hester RL. Exercise-induced increase in skeletal muscle vasodilatory responses in obese Zucker rats. Am J Physiol Regul Integr Comp Physiol. 2005; 288(4):R987-91.

43. Xiang L, Naik JS, Hodnett BL, Hester RL. Altered arachidonic acid metabolism impairs functional vasodilation in metabolic syndrome. Am J Physiol Regul Integr Comp Physiol. 2006; 290(1):R134-8. 
Table 1. Baseline characteristics of animal groups within the present study. Abbreviations: EX (exercise), CAP (captopril), HDZ (hydralazine), MET (metformin), RGZ (rosiglitazone), TEM (TEMPOL), ATOR (atorvastatin), GEM (gemfibrozil); * $\mathrm{p}<0.05$ vs. LZR; $\uparrow \mathrm{p}<0.05$ vs. OZR.

\begin{tabular}{|c|c|c|c|c|c|c|c|c|c|c|c|}
\hline & Weeks & $\begin{array}{l}\mathbf{L Z R} \\
(\mathrm{n}=8)\end{array}$ & $\begin{array}{c}\mathbf{O Z R} \\
(\mathrm{n}=8)\end{array}$ & $\begin{array}{l}\text { OZR- } \\
\text { EX } \\
(n=8)\end{array}$ & $\begin{array}{l}\text { OZR- } \\
\text { CAP } \\
(n=8)\end{array}$ & $\begin{array}{l}\text { OZR- } \\
\text { HDZ } \\
(n=8)\end{array}$ & $\begin{array}{l}\text { OZR- } \\
\text { MET } \\
(n=8)\end{array}$ & $\begin{array}{l}\text { OZR- } \\
\text { RGZ } \\
(\mathrm{n}=8)\end{array}$ & $\begin{array}{l}\text { OZR- } \\
\text { ATOR } \\
(n=8)\end{array}$ & $\begin{array}{l}\text { OZR- } \\
\text { GEM } \\
(\mathrm{n}=8)\end{array}$ & $\begin{array}{l}\text { OZR- } \\
\text { TEM } \\
(\mathrm{n}=8)\end{array}$ \\
\hline \multirow{2}{*}{$\begin{array}{c}\text { Mass } \\
(\mathrm{g})\end{array}$} & $7-8$ & $148 \pm 7$ & $340 \pm 8^{*}$ & $328 \pm 12$ & $338 \pm 9 *$ & $348 \pm 7 *$ & $331 \pm 8^{*}$ & $344 \pm 6^{*}$ & $340 \pm 11 *$ & $337 \pm 8^{*}$ & $351 \pm 6^{*}$ \\
\hline & $16-17$ & $308 \pm 9$ & $688 \pm 14 *$ & $570 \pm 14^{*}$ & $680 \pm 10^{*}$ & $679 \pm 12 *$ & $672 \pm 12 *$ & $681 \pm 7^{*}$ & $667 \pm 12 *$ & $374 \pm 10^{*}$ & $690 \pm 8^{*}$ \\
\hline \multirow{2}{*}{$\begin{array}{c}\text { MAP } \\
(\mathbf{m m H g})\end{array}$} & $7-8$ & $104 \pm 4$ & $102 \pm 5$ & $101 \pm 4$ & $98 \pm 4$ & $102 \pm 6$ & $97 \pm 6$ & $100 \pm 5$ & $100 \pm 5$ & $99 \pm 4$ & $102 \pm 6$ \\
\hline & $16-17$ & $105 \pm 6$ & $134 \pm 7 *$ & $124 \pm 6^{*}$ & $104 \pm 6 \dagger$ & $101 \pm 5 \dagger$ & $128 \pm 7^{*}$ & $120 \pm 5^{*}$ & $118 \pm 5^{* \dagger}$ & $125 \pm 5^{*}$ & $114 \pm 6^{* \dagger}$ \\
\hline \multirow{2}{*}{$\begin{array}{l}\text { Glucose } \\
\text { (mg/dl) }\end{array}$} & $7-8$ & $84 \pm 5$ & $88 \pm 5$ & $91 \pm 6$ & $92 \pm 6$ & $91 \pm 6$ & $88 \pm 7$ & $92 \pm 6$ & $92 \pm 6$ & $94 \pm 6$ & $92 \pm 4$ \\
\hline & $16-17$ & $94 \pm 4$ & $128 \pm 7^{*}$ & $114 \pm 8 *$ & $111 \pm 9$ & $125 \pm 7 *$ & $106 \pm 5 \dagger$ & $99 \pm 6 \dagger$ & $118 \pm 6^{*}$ & $125 \pm 8 *$ & $119 \pm 6^{*}$ \\
\hline \multirow{2}{*}{$\begin{array}{l}\text { Insulin } \\
\text { (ng/ml) }\end{array}$} & $7-8$ & $0.9 \pm 0.2$ & $5.8 \pm 0.5^{*}$ & $5.5 \pm 0.6^{*}$ & $5.5 \pm 0.4^{*}$ & $5.4 \pm 0.7 *$ & $5.4 \pm 0.5 *$ & $5.6 \pm 0.5^{*}$ & $5.7 \pm 0.6^{*}$ & $5.5 \pm 0.5^{*}$ & $5.2 \pm 0.4^{*}$ \\
\hline & $16-17$ & $1.3 \pm 0.2$ & $8.4 \pm 0.5^{*}$ & $5.8 \pm 0.7^{* \dagger}$ & $6.2 \pm 0.6^{* \dagger}$ & $8.0 \pm 0.7 *$ & $4.7 \pm 0.7 * \dagger$ & $4.2 \pm 0.5^{*} \dagger$ & $6.0 \pm 0.6^{*} \dagger$ & $6.5 \pm 0.6^{* \dagger}$ & $6.8 \pm 0.5^{*} \dagger$ \\
\hline \multirow{2}{*}{$\begin{array}{c}\text { N-tyrosine } \\
(\mathbf{n g} / \mathrm{ml})\end{array}$} & $7-8$ & $8 \pm 3$ & $17 \pm 3^{*}$ & $16 \pm 2 *$ & $16 \pm 5$ & $17 \pm 4$ & $15 \pm 5$ & $16 \pm 5$ & $18 \pm 4 *$ & $16 \pm 5$ & $17 \pm 4$ \\
\hline & $16-17$ & $14 \pm 4$ & $54 \pm 8 *$ & $35 \pm 5 * \dagger$ & $40 \pm 7 *$ & $47 \pm 7 *$ & $26 \pm 4 * \dagger$ & $24 \pm 5 \dagger$ & $38 \pm 6 * \dagger$ & $41 \pm 6^{*}$ & $22 \pm 6 \dagger$ \\
\hline
\end{tabular}




\section{FIGURE LEGENDS}

Figure 1. Correlations between plasma levels of cortisol and aggregate coat score in male LZR and OZR between age ranges 7-8 weeks and 16-17 weeks. Data are presented from individual animals under untreated conditions (control; Panel A) and following all chronic interventions (exercise, anti-oxidant, anti-hypertension, anti-diabetes, anti-dyslipidemia; Panel B) The line of best fit through the data are is also presented for each relationship. Please see text for details.

Figure 2. Correlations between aggregate coat score and the key outcome parameters in the present study for male LZR and OZR between age ranges 7-8 weeks and 16-17 weeks. Data are presented from individual animals versus the upper bound of the acetylcholine concentration-response curve (ACh Upper Bound; Panels A or E), the index of glycemic control (HOMA; Panels B or F), the index of oxidant stress/inflammation (Oxidant Load; Panels $\mathrm{C}$ or $\mathrm{G}$ ) and the index of arachidonic acid metabolism (AA Metab; Panels D or $\mathrm{H}$ ) in untreated control conditions or following chronic interventions, respectively. The line of best fit through the data is also presented for each relationship $\left(r^{2}>0.75\right.$ for all). Please see text for details.

Figure 3. Correlations between plasma cortisol levels and the key outcome parameters in the present study for male LZR and OZR between age ranges 7-8 weeks and 16-17 weeks. Data are presented from individual animals versus the upper bound of the acetylcholine concentration-response curve (Ach Upper Bound; Panels A or E), the index of glycemic control (HOMA; Panels B or F), the index of oxidant stress/inflammation (Oxidant Load; Panels $\mathrm{C}$ or $\mathrm{G}$ ) and the index of arachidonic acid metabolism (AA Metab; Panels D or H) in untreated control conditions or following chronic interventions, respectively. The line of best fit through the data are is also presented for each relationship. Please see text for details.

Figure 4. Graphical representation of the normal relationships between the dilator reactivity of skeletal muscle resistance arterioles to increasing concentrations of acetylcholine, plasma cortisol concentration and aggregate coat score in untreated (control) male LZR and OZR between age ranges 7-8 weeks and 16-17 weeks (Panel A). 
The raw data from each animal are fit with a smoothing function that integrates a running average between near neighbor data points. Panel B presents this relationship from each animal within a group following the chronic interventions utilized in the present study. Panels C and D, represent the relationships in the untreated (control) LZR and OZR and divides the interventions into those that were effective at improving vascular and behavioral outcomes (Panel C) and those that were not (Panel D), using the same smoothing function as for Panel A. Please see text for details.

Figure 5. Data describing the ability of imposed chronic interventions of the present study to restore normal levels of the key outcome parameters in LZR and OZR in the present study. Data are presented for the effectiveness of the imposed interventions on coat score (Panel A), plasma cortisol (Panel B), vascular reactivity (Panel C), glycemic control (Panel D), oxidant stress/inflammation lad (Panel E), and arachidonic acid metabolism (Panel F). The imposed chronic interventions were exercise (EX), captopril (CAP), hydralazine (HDZ), metformin (MET), rosiglitazone (RGZ), atorvastatin (ATOR), gemfibrozil (GEM) and TEMPOL (TEM). The horizontal line in each panel represents an improvement of $30 \%$ from the maximum degree of dysfunction for that parameter. 

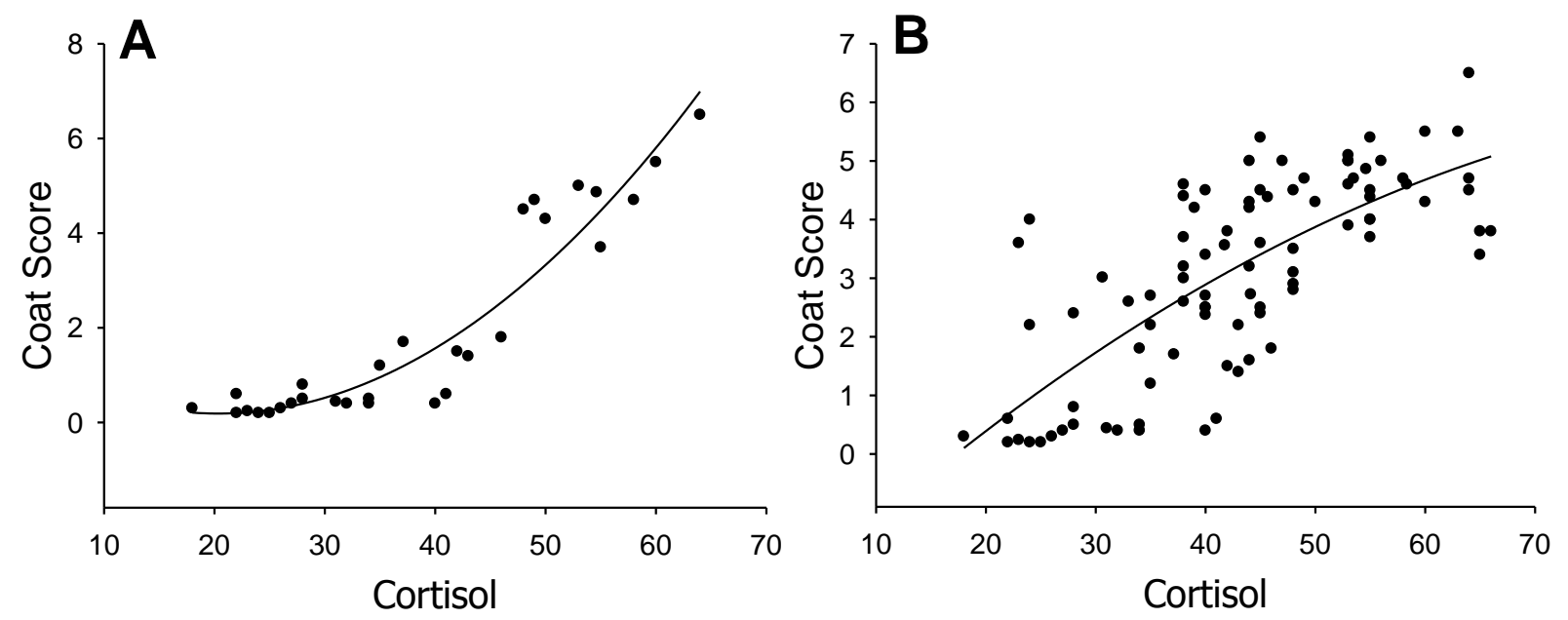

Figure 1.

Stanley et al. 

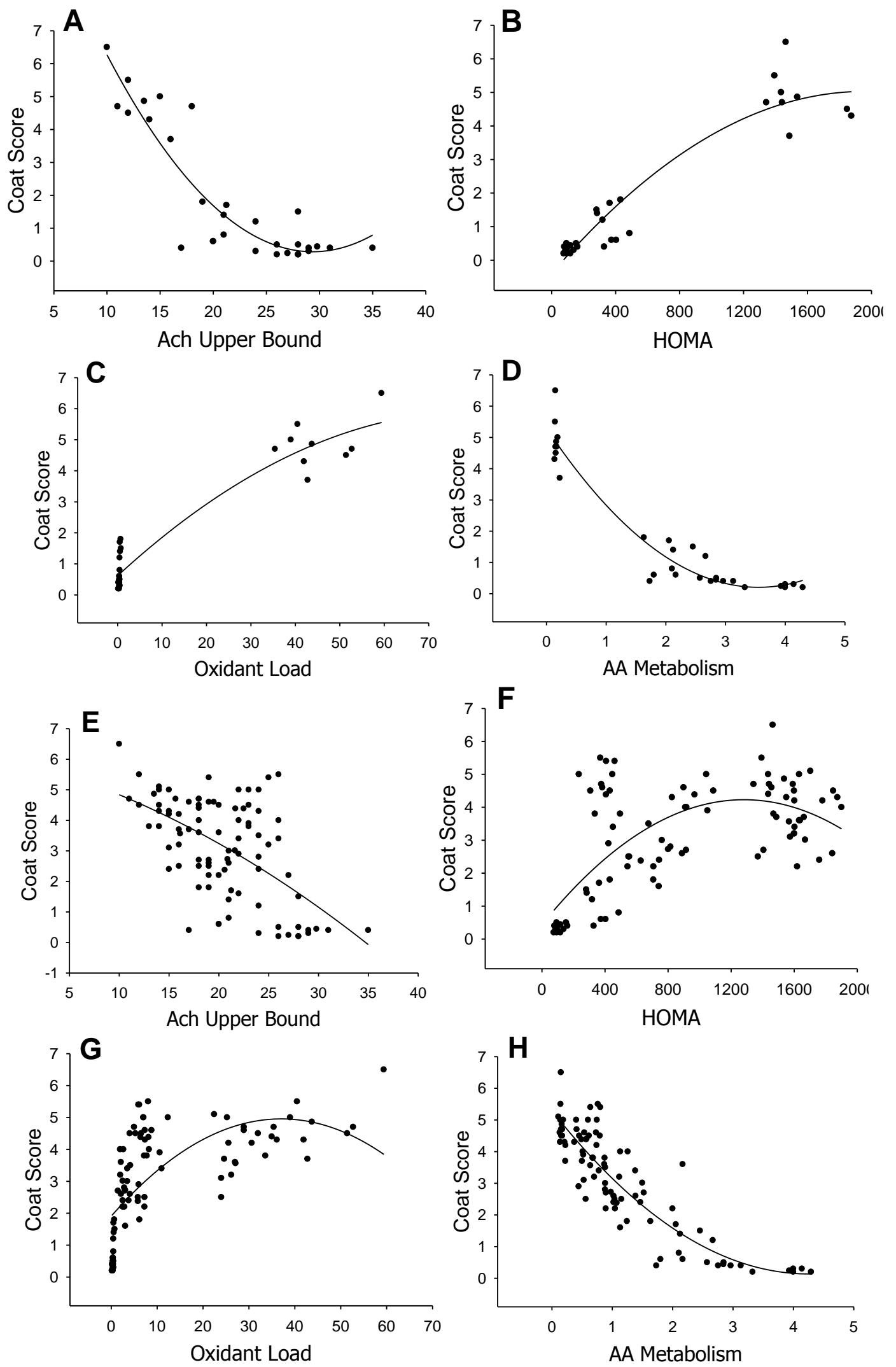

Figure 2.

Stanley et al. 

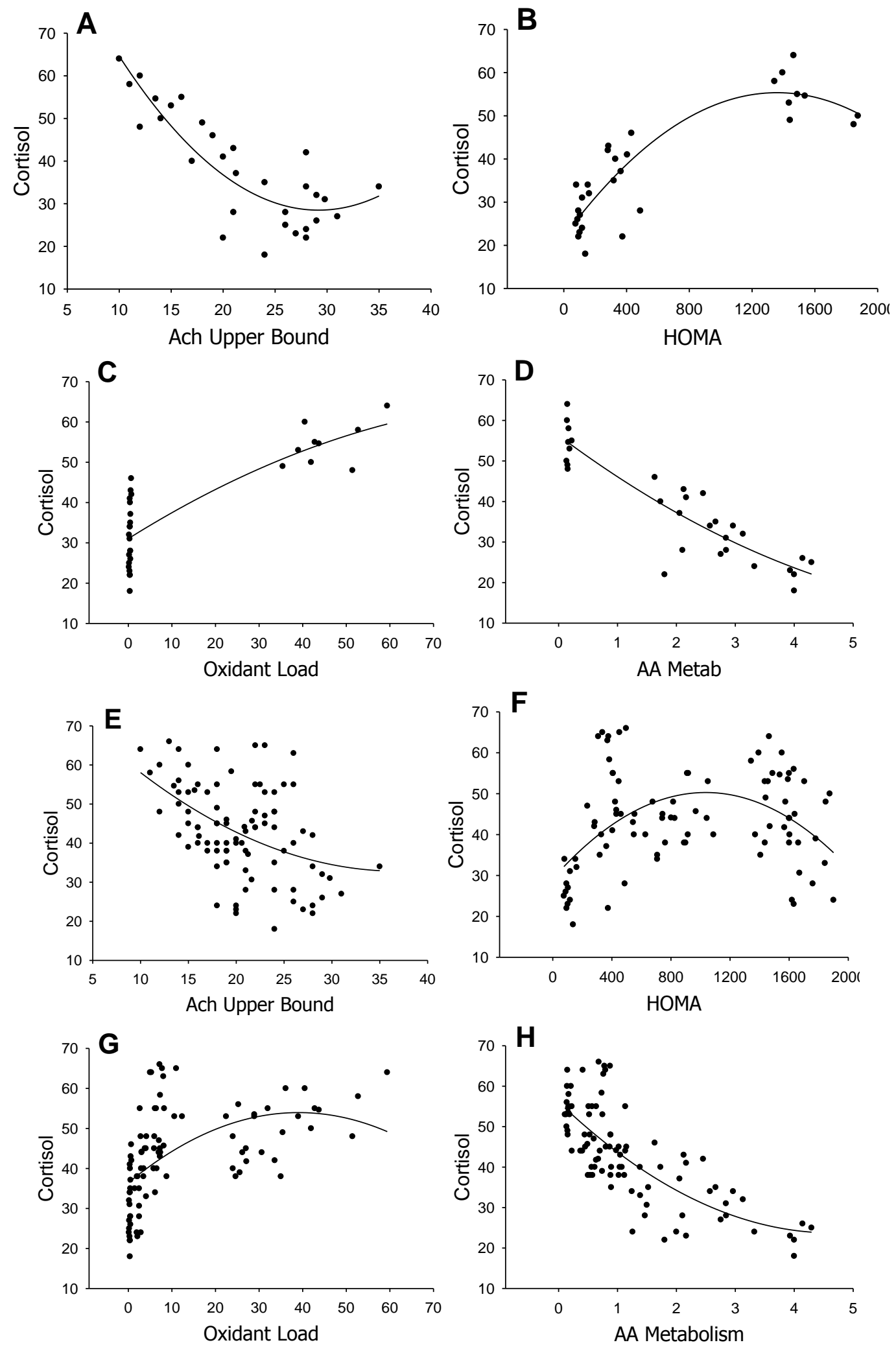

Figure 3.

Stanley et al. 

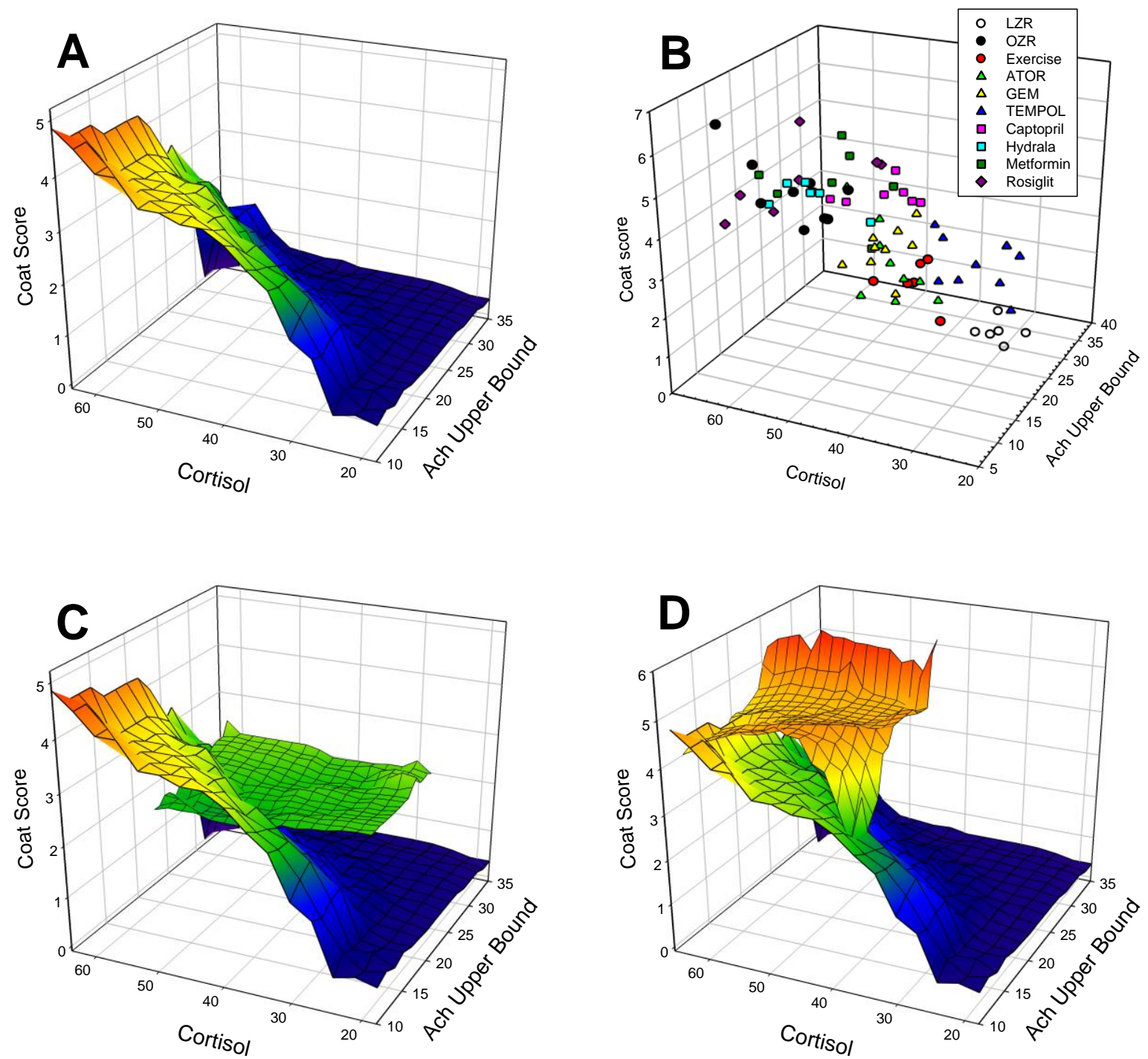

Figure 4.

Stanley et al. 


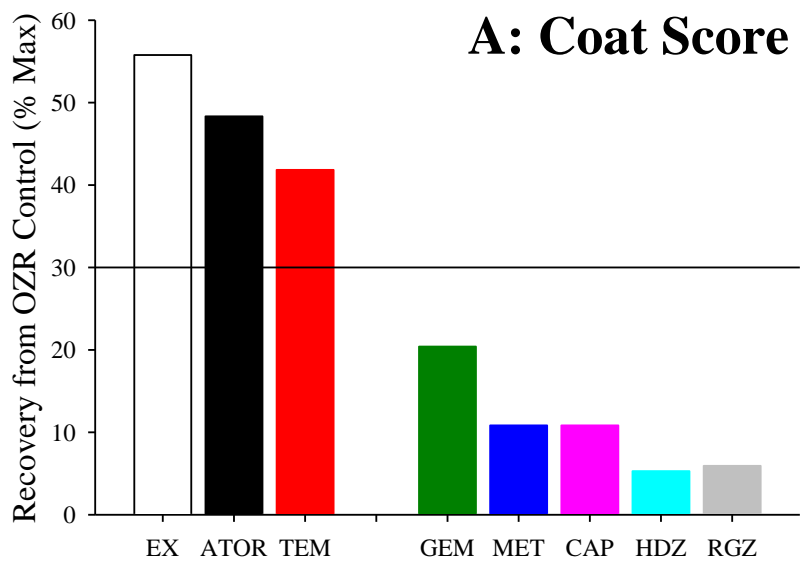

Experimental Intervention

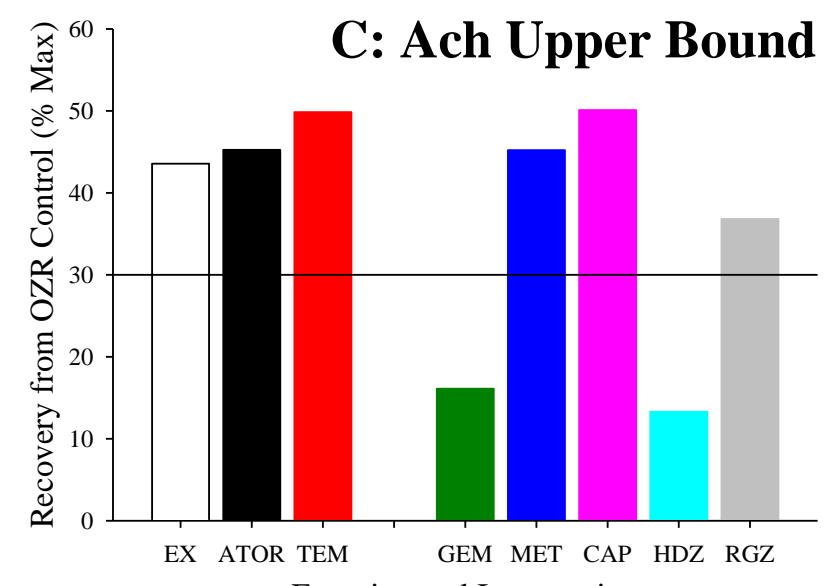

Experimental Intervention

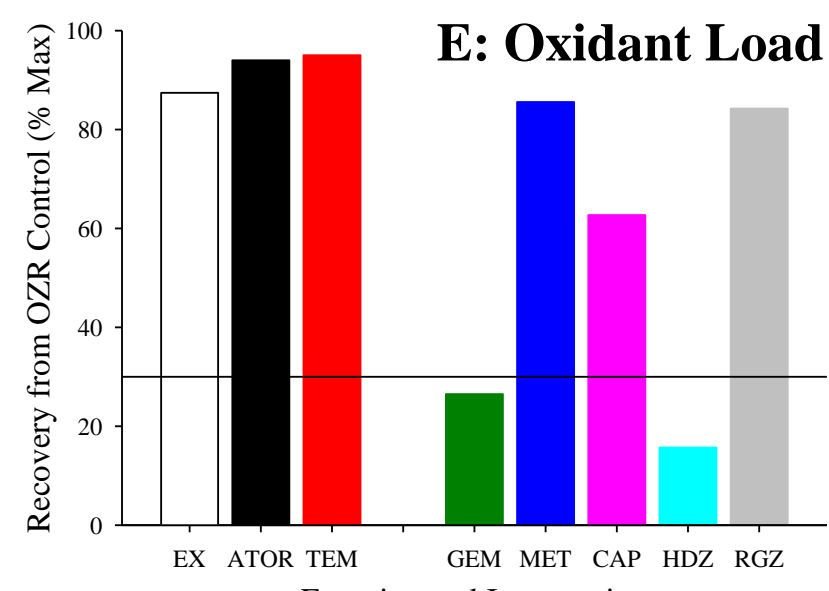

Experimental Intervention

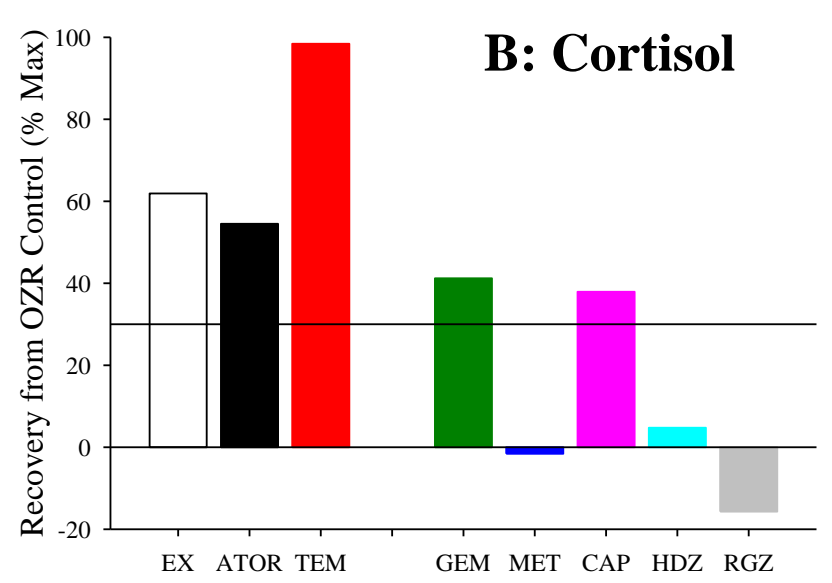

Experimental Intervention

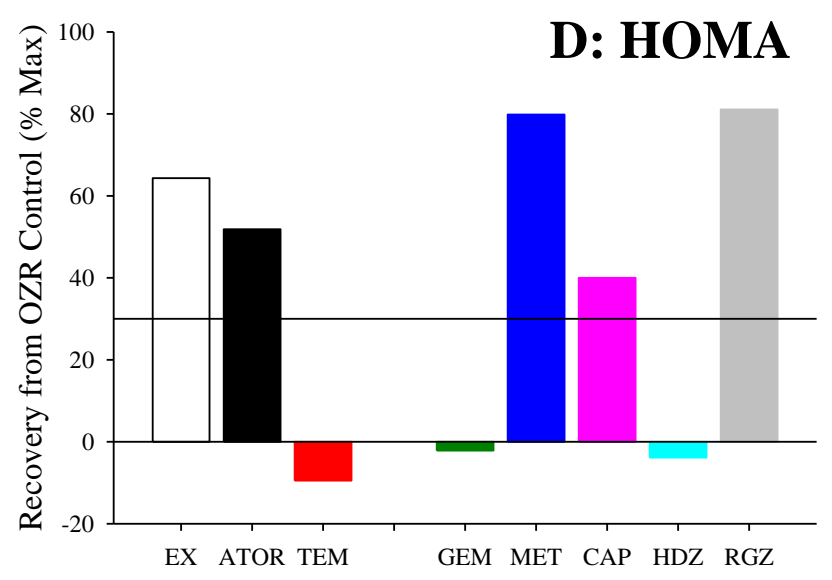

Experimental Intervention

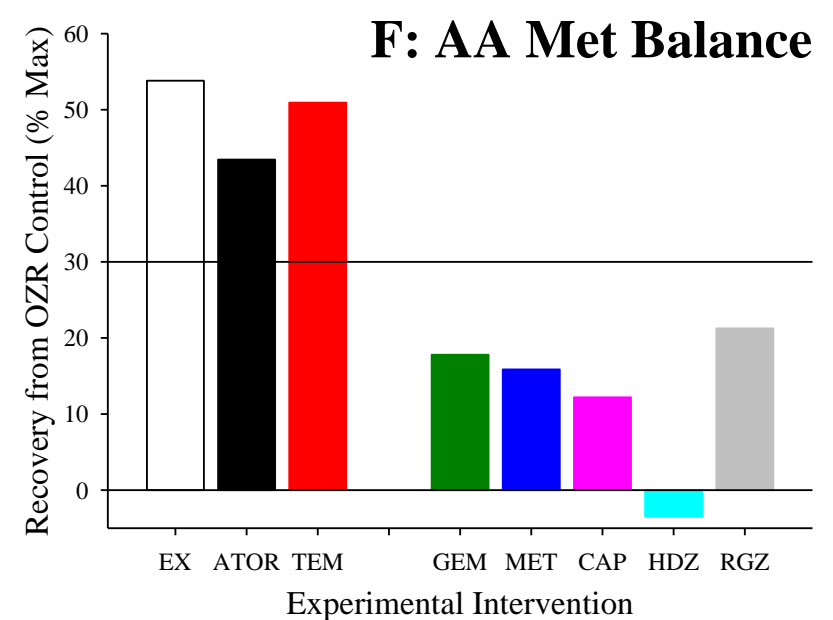

Experimental Intervention

Figure 5.

Stanley et al. 


\section{CHAPTER 3: CONCEPTUAL FRAMEWORK}

This study sought to address whether controlling inflammation and oxidant stress through either the use of an exercise regimen or chronic pharmacologic treatments would result in improved vascular function and behavioral outcomes. Several key findings emerged from this study:

- Exercise training and two pharmacological treatments- Atorvastatin and TEMPOL- were able to improve coat score, as well as reduce cortisol levels. Levels of cortisol corresponded strongly with behavioral outcomes in OZR animals. As cortisol is the main hormone of the stress response, the strong relationship between cortisol and behavioral outcome was expected, and the reduction of cortisol from treatments can therefore be taken as the most significant link towards improved behavioral outcomes.

- Several of the treatments- exercise, Atorvastatin, TEMPOL, Metformin, Captopril, and Rosiglitazone were all able to improve the oxidant load in OZRs, as well as improve endothelium-dependent dilation to ACh. These findings support our previous observations that improving NO bioavailability by reducing oxidant stress results in improved vasodilation to ACh. However, neither improved vascular reactivity nor improved oxidant load were correlated with improved coat scores and lowered cortisol. Exercise, TEMPOL, and Atorvastatin each were able to improve both vascular function and behavioral outcomes, while metformin, rosiglitazone, and Captopril had no impact on behavior or cortisol. These findings indicate that reduction of oxidant stress and improvement of NO bioavailability alone are insufficient to improve behavior and reduce cortisol.

- Rosiglitazone and metformin both treat glycemic management, and indeed were seen in this study to improve HOMA; however, these drugs did not improve coat score or cortisol. Captopril controls hypertension, and was able to reduce oxidant load and strongly improve endothelial function, but was also ineffective at improving behavior and cortisol. These findings indicate that improved metabolic control and management of blood pressure are not sufficient to improve behavior or cortisol.

- The three interventions that effectively improved behavior- Atorvastatin, TEMPOL, and Exercise- were also unique in that they were the only three that improved AA metabolism balance, indicating a shift towards production of $\mathrm{PGI}_{2}$ and away from $\mathrm{TxA}_{2}$ production. These findings indicate that either improved AA metabolism alone, or perhaps improved AA metabolic balance coupled with attenuated oxidative stress, may be key 
components mediating behavioral outcomes and cortisol levels in the OZR model of metabolic syndrome. Improving oxidant load or HOMA alone was not related to AA metabolism, suggesting that improvements in this metric were an independent effect of exercise, TEMPOL, and Atorvastatin.

Overall, the results of this study indicate that interventions which both reduce levels of oxidant stress and promote an anti-inflammatory shift in COX metabolism are most effective at improving coat score and reducing levels of cortisol. Improving oxidant stress and vascular reactivity alone is not sufficient to improve depressive symptoms. A future expansion of this study should evaluate the prophylactic efficacy of Atorvastatin, exercise, and TEMPOL towards preventing the induction of depressive symptoms in animals undergoing UCMS. 


\section{DISCUSSION}

The purpose of this dissertation was to investigate the effects of chronic stress/depression on behavioral outcomes and vascular function in rodents in order to elucidate the underlying mechanisms linking depression incidence with cardiovascular development/outcomes. Animal models of depression are valuable research tools for investigating the mechanistic contributors and functional outcomes linking depression with vascular disease. The fundamental concept of the UCMS protocol is that exposure to chronic, uncontrollable, environmental stressors induces behavioral, psychological, and physiological changes that alter homeostatic balance and the ability to adapt to subsequent stress challenges. Early work from the laboratory of Catherine Belzung and from our laboratory demonstrated that UCMS promoted vascular pathologies in healthy BALBc/J mice, and concurrent studies have confirmed variable responses based on sex. As women are twice as likely to develop depression compared to age matched men, preliminary data from initial experiments showed a similar trend in which female rodents were more susceptible to UCMS-induced behavioral changes versus their male counterparts, thereby leading to our initial exploration of sex differences utilizing the UCMS model.

The first chapter of this dissertation emphasized the variable effects of UCMS on behavioral and physiological outcomes between male and female BALB/cJ mice. The results showed that compared to UCMS males, UCMS female animals exhibited a greater susceptibility to behavioral symptoms as measured by worsening of the coat score, reduced sucrose-stimulated grooming, and increased immobility in the tail suspension test, as well as increased plasma markers of oxidant stress, inflammation and HPA dysfunction. However, despite these elevated responses, the developed vasculopathy in both the conduit and resistance vasculature of female mice was less severe than that of males as evidenced by superior maintenance of endothelium-dependent vasodilator response to agonist challenge in aortic rings and gracilis arterioles. Much of this blunted impairment appeared to be a function of a superior maintenance of endothelial function, primarily via more modest reduction of NO bioavailability and more favorable balance in arachidonic acid metabolism. Together, these data indicate a greater capacity of females to attenuate the negative effects of chronic stress/depressive symptoms. Importantly, similar responses were observed between individual female rodents despite the fact that they were randomized with respect to the time of their estrous cycles, thus vascular and 
behavioral outcomes were not associated with observable differences in hormonal fluctuations, suggesting that the protective effects may confer a more stable, long standing feature of female physiology. This study provided a foundation for further investigation into the potential mechanisms involved in the sex disparity related to differences in prevalence, severity, and outcomes of depression and CVD.

Building on this work, the second chapter investigated the concept of "female protection" from chronic stressinduced effects on adverse vascular health and changes in these outcomes in ovariectomized females. Two important alterations in experimental approach of Chapter 2 improved the translational relevance of this study: use of rats, as this species has a more robust background in peripheral vascular disease research as well as a more comparable CVD pathology to that of human disease; and expansion of the study to include a model of comorbid depression and CVD. This approach provides mechanistic insight into the integrative system of comorbid conditions that have an additive effect in promoting adverse CV outcomes. Chapter 2 investigates comorbid CVD risk and depression in the obese Zucker rat, a model of metabolic syndrome, and the effects of loss of female sex hormones in OVX females, and the potential overlap of these effects on UCMS induced depressive like behavior and vascular reactivity.

Imposition of UCMS in LZR resulted in similar sex differences in the development of depressive behaviors, as well as impairment of vasodilator response demonstrated by results of chapter 1 . An unexpected development of depressive behaviors occurred in male and female OZR in the absence of chronic stress, suggesting that the genetic make-up of OZR predispose these animals to depressive symptoms, or alternatively, that the development of behavioral changes is induced by the progressive worsening of metabolic syndrome. Regardless, worsened coat scores and depressive behaviors in OZR have been previously observed in unpublished work from our laboratory, and the quantification of depressive behaviors in the non-stressed OZR group provides evidence that the OZR itself may be a suitable model for studying CVD-related depression. The imposition of chronic stress induced elevated levels of inflammatory biomarkers and oxidative stress in addition to the damaging effects of dysfunctional lipid profiles and increased adiposity associated with metabolic syndrome in the OZR model. Our results clearly showed that pre-existing CVD amplified the deleterious vascular effects of UCMS, and eliminated the sex-specific vascular protection observed in UCMS female LZR. 
Finally, these outcomes were investigated in ovariectomized (OVX) female animals. OVX attenuated behavioral impairment to UCMS, elevated plasma glucose and inflammatory markers, and impaired vascular endothelial dependent dilator responses, specifically due to increased pro-inflammatory factors, loss of NO bioavailability, and impaired endothelial reactivity. Interestingly, loss of estrogen promoted these responses in LZR with or with-out chronic stress, while OVX alone did not alter vascular response in OVX OZR relative to intact OZR control. An increased risk in CVD is strongly associated with menopause in women, and these results support the hypothesis that the loss of sex hormones, perhaps in conjunction with the psychological and social stressors associated with menopause, contributes to the development of vasculopathy. Together, this study suggests that loss of estrogen-based protection against inflammation and oxidative stress enhances risk of endothelial dysfunction in lean females, while loss of estrogen in female OZR enhanced the vascular dysfunction only in conditions of co-morbid CVD and depression. Overall, the results of the second chapter of this dissertation strongly support the theory that inflammation and oxidative stress are significant contributing factors to the development of depressive behaviors and vascular impairments in both male and female, and lean and obese, animals, and that endogenous female sex hormones in lean animals play a significant role protecting the vasculature against these factors.

The final chapter of this dissertation is built upon key observation gathered from the first two chapters linking inflammation and oxidative stress with depression and vascular dysfunction. This raises the possibility that treating these parameters to improve vascular health may also prove effective in attenuating depressive symptoms. Chronic treatment using common pharmaceutical drugs targeting components of metabolic syndrome including: hypertension (captopril), peripheral vasoconstriction (hydralazine), glucose regulation (metformin), insulin insensitivity (rosiglitazone), hypercholesterolemia (atorvastatin), and dyslipidemia (gemfibrozil), were used in addition to TEMPOL and exercise training, to assess the efficacy in improving vascular function as well as depressive behaviors (coat score and plasma cortisol).

The normal relationship between vascular function, cortisol and coat score between LZR and OZR clearly indicated that only exercise, atorvastatin, and TEMPOL, were able to shift responses towards improved vascular function as well as lower cortisol and reduced coat score, thus attenuating depressive symptom severity. These 
three interventions were also unique in their ability to improve AA metabolism balance, thus favoring a shift towards production of $\mathrm{PGI}_{2}$ over $\mathrm{TxA}_{2}$. Overall, the results of Chapter 3 indicate that interventions that are most effective at improving oxidant stress and inflammation are also most effective at decreasing coat score and reducing levels of cortisol. Improving oxidant stress and vascular reactivity alone is not sufficient to improve depressive symptoms, suggesting that behavioral outcomes of UCMS are likely related to both an increase in oxidative stress and inflammation. It is possible that prophylactic efficacy of Atorvastatin, exercise, and TEMPOL or some combination of the three may therefore attenuate severity of depressive symptoms with UCMS. Of note, previous work from our laboratory (Frisbee et al., 2011) revealed that functional impairments in skeletal muscle performance in OZR are the combined result of increased $\alpha$-adrenergic signaling, oxidative stress, and TxA $\mathrm{A}_{2}$ production, and only synergistic treatment of these factors restored skeletal muscle function in OZR. Interestingly, the same factors (inflammation, oxidative stress, and $\mathrm{TxA}_{2}$ production, as well as adrenergic signaling) are present in LZR following UCMS, and were further increased in OZR with UCMS. This commonality points to a larger conceptual framework of CVD pathophysiology, in which a disturbance in the healthy homeostatic balance (e.g. hypertension, metabolic syndrome, chronic stress/depression, etc.) affects mechanisms regulating vascular tone and endothelial function, with functional consequences on tissue perfusion in the periphery and development of depressive behaviors. Additionally, the combination of multiple risk factors would therefore predict an accelerated and advanced development of vascular dysfunction involving reduced NO bioavailability, increased $\mathrm{TxA}_{2}$ production, pro-oxidative, pro-inflammatory conditions, and enhanced adrenergic tone. The work detailed in this dissertation provides compelling evidence in support of such a framework, and demonstrates that progressive treatment of CVD that targets one component is largely ineffective due to inability to restore homeostatic balance in the vasculature.

\section{Clinical Significance}

Based on the data from this dissertation, a compelling case can be made for a causal relationship between depression and CVD. Clinical and epidemiological studies have consistently demonstrated a strong association between depression and cardiovascular morbidity and mortality, with profound differences in clinical presentation and symptom severity between males and females. Depression has been linked to impairments in vascular and 
endothelial functions, and the roles of inflammation and oxidative stress have been consistently reported as strong potential mediators (Fiedorowicz, 2014). However, it has been argued that these components explain only a fraction of the complex integrated relationship between depression and vascular outcomes (Vaccarino et al., 2007); concurrently, a single biomarker measurement is not able to adequately describe symptom severity or the variable clinical presentations between sexes, findings that were clearly evident from the experiments outlined in this dissertation. This underscores one of many challenges that limit the ability for researchers to effectively address this important public health issue.

Treatment of depression with currently available antidepressant therapies, most commonly SSRIs, has demonstrated variable clinical effects and limited efficacy, with successful therapeutic effects in less than 50 percent of patients, and in patients that respond initially, a high percentage experience a relapse or recurrence of depressive episodes after discontinuing treatment (Brosse et al., 2002). Furthermore, antidepressants have not been established as a means of reducing associated risk of CVD, and may have adverse effects on CV health. While SSRIs increase levels of serotonin within synapses in the brain, their main anti-depressant effect is thought to be linked to increasing neurogenesis within the hippocampus (Clayton, 2012). Our results are consistent with other preclinical reports that suggest that inflammation and oxidative stress are linked to depressive symptoms (Patki et al., 2015; Xu et al., 2014); we hypothesize that it is therefore possible that a cerebral microenvironment hostile to neurogenesis may decrease the efficacy of many antidepressant drugs. We can speculate that targeting both increasing serotonin levels and reducing the oxidant load within in the brain could increase the efficacy of popular SSRIs and other antidepressants. For example, the common statin drug, atorvastatin, a 3-hydroxy-3methylglutarylcoenzyme A (HMGCoA) reductase inhibitor widely used in clinical practice as a lipid-lowering agent, has also been reported to have antidepressant effects (Shahsavarian et al., 2014) (similar to the results shown in Chapter 3). Although the exact mechanisms are not known, protective effects of statins have been linked to their role as immunomodulatory agents against oxidative stress and inflammation. In addition, data have indicated that atorvastatin may directly influence neuronal function by modulating important neuropeptides as well as essential neurotransmitter systems, including serotonergic activity, involved in the pathophysiology of depression (Ludka et al., 2014). Taken together, it is possible that coupling antidepressants with Atorvastatin or a 
more stable form of an antioxidant drug like TEMPOL could improve treatment efficacy and reduce disease burden in many patients with depression. Alternatively, Atorvastatin or TEMPOL alone may be effective at improving depressive symptoms in patients who have comorbid metabolic syndrome or CVD, suggesting that they possess pleiotropic protective effects in the central nervous system that are associated with depression that may be independent of their main function on vascular factors.

However, treatment of comorbid CVD and depression may be challenging due to the high complexity of medications with pleiotropic effects in comorbid diseases. Therefore, non -pharmacological interventions, such as exercise training, have been reported as successful treatments for depression (Rong-Hui et al., 2014; Craft et al., 2004). Theoretically, exercise may provide important advantages over pharmacotherapy, including fewer drug interactions and more involvement of patients in their self-care. Furthermore, reports have shown that exercise training is as effective as most antidepressants at improving depression symptoms and reducing the incidence of recurrent episodes (Craft et al., 2004); it has been demonstrated to lead to significant improvements of adverse pathophysiological effects of depression, including lowered sympathetic nervous system activity, increased heart rate variability, decreased inflammation, improve CNS functioning, and promote endothelial function (Rong-Hui et al., 2014).

The significance of this research has far reaching clinical implications, especially as the high prevalence of comorbid depression and CVD or CV risk factors, including MetS and its components, continue to rise, resulting in amplification of the deleterious health outcomes that are more complicated and burdensome than the individual condition itself. Clinicians should therefore strongly consider monitoring patients with depression for CVD, and vice versa. Additionally, future studies are needed to better understand the specific mechanisms by which elevated risk of depression itself can become an evidence-based target for intervention on cardiovascular risk, ideally investigating potential treatment interventions that concomitantly improve both depression and cardiovascular risk or risk factors, such as Atorvastatin and TEMPOL. This should be an impetus for prioritizing the improved integration of behavioral and medical care. 


\section{REFERENCES}

1. Vaccarino V, Johnson BD, Sheps DS, Reis SE, Kelsey SF, Bittner V, et al. Depression, inflammation, and incident cardiovascular disease in women with suspected coronary ischemia: the National Heart, Lung, and Blood Institute-sponsored WISE study. J Am Coll Cardiol. 2007; 50(21):2044-50.

2. Fiedorowicz JG. Depression and Cardiovascular Disease: An Update on How Course of Illness May Influence Risk Current Psychiatry Reports.2014; 16:492.

3. Rong-Hui T, Zhi-Yu Z, Guo-Qiang Z, Wei-Feng W, Yun-Juan L, Zhan-Dong B, Yan H, Wei-Qiang H, and LiMei Y. Effects of exercise training on depression in patients with heart failure: a systematic review and metaanalysis of randomized controlled trials. European J of Heart Failure.2014; 16:749-757.

4. Brosse AL, Sheets ES, Let HS, Blumenthal JA. Exercise and the Treatment of Clinical Depression in Adults Recent Findings and Future Directions Sports Med. 2002; 32(12):741-760.

5. Clayton AH. Understanding antidepressant mechanism of action and its effect on efficacy and safety. Journal of Clinical Psychiatry. 2012. 73(3):e11.

6. Patki G, Salvo A, Li H, Atrooz F, Isam A, Kelly M, Salin S. Tempol Treatment Reduces Anxiety-Like Behaviors Induced by Multiple Anxiogenic Drugs in Rats. Plos One. 2015; 10(3):e0117498.

7. Xu Y, Wang C, Klabnik JJ, O’Donnel JM. Novel Therapeutic Targets in Depression and Anxiety: Antioxidants as a Candidate Treatment. Current Neuropharmacology. 2014; 12(2):108-119.

8. Shahsavarian A, Javadi S, Jahanabadi S, Khoshnoodi M, Shamsaee J, et al. Antidepressant-like effect of atorvastatin in the forced swimming test in mice: the role of PPAR-gamma receptor and nitric oxide pathway. European Journal of Pharmacology. 2014; 745:52-8.

9. Ludka FK, Constantino LC, Kuminek G, Binder LB, Zomkowski AD et al. Atorvastatin evokes a serotonergic system-dependent antidepressant-like effect in mice. Pharmacology Biochem and Behavi. 2014; 122:253-60. 
10. Craft LL, Perna FM. The Benefits of Exercise for the Clinically Depressed. Primary Care Companion- Journal of Clinical Pyschiatry. 2004; 6(3):104-111. 